\title{
Characterization of the Dynein-Dynactin Interaction
}

\author{
Dissertation \\ zur Erlangung des \\ mathematisch-naturwissenschaftlichen Doktorgrades \\ "Doctor rerum naturalium" \\ der Georg-August-Universität Göttingen
}

vorgelegt von

Peggy Findeisen

aus Grimma

Göttingen 2014 
Thesis Committee:

PD Dr. Martin Kollmar (Reviewer)

Research Group Systems Biology of Motor Proteins

Max Planck Institute for Biophysical Chemistry, Göttingen

Prof. Reinhard Lührmann (Reviewer)

Department of Cellular Biochemistry

Max Planck Institute for Biophysical Chemistry, Göttingen

Dr. Hans Dieter Schmitt

Research Group Membrane Transport in Yeast

Max Planck Institute for Biophysical Chemistry, Göttingen

Date of oral examination: August $1^{\text {st }}, 2014$ 
I hereby declare that this thesis was written independently and with no other sources and aids than quoted.

Göttingen, June 30th, 2014

Peggy Findeisen 



\section{Talks}

2012, June

GGNB Biomolecules Retreat, St. Andreasberg, Germany

Structural Characterization of the Dynein-Activator, Dynactin

\section{Poster presentation}

2012, September

14th International Conference on the Crystallization of Biological Macromolecules;

Huntsville, USA

Structural Characterization of the Dynein-Dynactin Interaction

2013, March

Alpbach Workshop on Molecular Motors 2013; Alpbach, Österreich

Structural Characterization of the Dynein-Dynactin Interaction

2013, December

GGNB Science Day, Göttingen, Germany

Structural Characterization of the Dynein-Dynactin Interaction 



\section{Abstract}

In eukaryotic cells many biological processes like cell division, the distribution of vesicles and organelles require the dynamic rearrangement of molecules and cellular components. These rearrangements are controlled by three superfamilies of motor proteins, which move along the cytoskeletal tracks. One of these motors is the cytoplasmic dynein complex, which exclusively moves along microtubules towards the minus-end thereby transporting cargo from the cell periphery back to the cell centre (retrograde transport). In order to adapt to the diverse cargoes dynein interacts with various proteins. One of them is dynactin, a large complex that is involved in almost every dynein function. The interaction of both complexes occurs between the N-terminus of two dynein intermediate chains (IC) and a coiled-coil region in the dynactin DCTN1 dimer.

For the characterization of the dynein-dynactin interaction chicken DCTN1 $1_{412-533}$ and human N-terminal IC were expressed in tandem in E. coli. Several constructs were designed in which the IC length varied between 47 and 84 amino acids. Crystallization trials were performed and a condition was found for growing large crystals with edge lengths of 100-200 $\mu \mathrm{m}$. X-ray measurements were performed at PETRA III (DESY, Hamburg) and the best native crystal diffracted to $2.9 \AA$. Phases were obtained at 3.4 Ausing the single anomalous diffraction approach and crystals consisting of selenomethionine-incorporated protein. A model of $\mathrm{DCTN1} 1_{412-533}$ was built by iterative cycles of manual model building and refinement of the model against the diffraction data. Surprisingly, the asymmetric unit of the crystal only contained DCTN1 $1_{412-533}$ in coiled-coil conformation without bound IC molecules. The DCTN1 $1_{412-533}$ structure belongs to the canonical parallel left-handed coiled-coil. Its surface is mainly negatively charged suggesting an electrostatical interaction with IC, which exhibits an alkaline pI. Analyses of the N-terminal IC sequence supported an assumption that IC might not bind as a coiled-coil but rather as separate $\alpha$-helices onto DCTN1. Cross-linking studies were performed with $\mathrm{BS}^{3}$ to narrow down the IC binding site on DCTN1. Two binding regions could be determined, which are mainly negatively charged.

This is the first structural insight into the DCTN1 $1_{412-533}$ coiled-coil. Based on the crosslinking experiments and the assumption of two single ICs binding onto DCTN1 several binding models were determined and analysed. A possible model is the interaction of two IC molecules, which bind parallel to both DCTN1 bindings sites. A parallel/antiparallel interaction of the IC molecules is also conceivable. The localization of the dynein-dynactin interaction could be further refined compared to previous studies. 



\section{Contents}

Abstract vii

$\begin{array}{ll}\text { List of used abbreviations } & 1\end{array}$

1. Introduction 3

1.1. Motor proteins . . . . . . . . . . . . . . . 3

1.2. Dynein . . . . . . . . . . . . . . . . . . . 4

1.2.1. Structure of cytoplasmic dynein . . . . . . . . . . . . . . . 4

1.2.2. Functions of cytoplasmic dynein . . . . . . . . . . . . . . 7

1.3. The activator of dynein: dynactin . . . . . . . . . . . . . 7

1.4. Aim of this work . . . . . . . . . . . . . . . . . . . 10

2. Materials and methods 13

2.1. Chemicals and equipment . . . . . . . . . . . . . . 13

2.2. Strains, plasmids and oligonucleotides . . . . . . . . . . . . . . . 13

2.3. Cloning techniques . . . . . . . . . . . . . . . . 16

2.3.1. Polymerase chain reaction (PCR) . . . . . . . . . . . . 16

2.3.2. PCR purification . . . . . . . . . . . . . . 16

2.3.3. $\quad$ Restriction digestion of DNA . . . . . . . . . . . . . 16

2.3.4. Agarose gel electrophoresis . . . . . . . . . . . . . . . . . 16

2.3.5. Gel extraction of DNA fragments . . . . . . . . . . . . 17

2.3.6. Ligation of DNA fragments . . . . . . . . . . . . 17

2.3.7. Plasmid purification . . . . . . . . . . . . . . . 17

2.3.8. DNA sequencing . . . . . . . . . . . . . . . 17

2.4. Transformation and cultivation of Escherichia coli . . . . . . . . . . . . 18

2.4.1. Transformation via heatshock . . . . . . . . . . . . . . 18

2.4.2. Cultivation media . . . . . . . . . . . . . . . . . . . . . 18

2.4.3. Synthesis of selenomethionine-containing proteins . . . . . . . 18

2.5. Protein expression test . . . . . . . . . . . . . . . . . . . . . . . 19

2.6. Protein purification $\ldots \ldots \ldots \ldots$

2.7. TEV-cleavage to remove His-tag . . . . . . . . . . . . . . . . . . . . . . 21 
2.8. Crystallization approach $\ldots \ldots \ldots$. . . . . . . . . . . . . . 22

2.8.1. Crystal seeding . . . . . . . . . . . . . . . . 22

$2.8 .2 . \quad$ Additive screening . . . . . . . . . . . . . . . . . 22

2.9. Crystal measurement . . . . . . . . . . . . . . . . . . 23

2.10. Structure determination . . . . . . . . . . . . . . . . . . . 23

2.10.1. Processing the datasets . . . . . . . . . . . . . . 23

2.10.2. Phase determination . . . . . . . . . . . . . . . . . . 24

2.10.3. Computing a starting model with $A C M I$. . . . . . . . . . . . . 24

2.11. Chemical cross-linking with $\mathrm{BS}^{3} \ldots \ldots \ldots$. . . . . . . . . 24

\begin{tabular}{lr}
\hline 3. Results & 27
\end{tabular}

3.1. Cloning and protein purification of various dynein-dynactin constructs. 27

3.1.1. $\mathrm{DCTN1}_{412-533}-\mathrm{IC}_{2-47} \ldots \ldots \ldots \ldots . \ldots . \ldots . \ldots 27$

3.1.2. $\mathrm{DCTN1}_{412-533}-\mathrm{IC}_{2-84} \ldots \ldots \ldots \ldots \ldots$

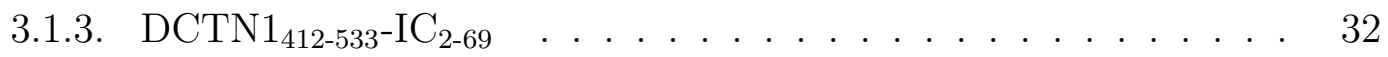

3.2. Crystallization of the various DCTN1-IC constructs and data collection 33

3.2.1. Crystallization of DCTN1 ${ }_{412-533}-\mathrm{IC}_{2-47}$ construct . . . . . . . . 33

3.2.2. Crystallization of DCTN1 ${ }_{412-533}-\mathrm{IC}_{2-47}$ with incorporated selenomethionine . . . . . . . . . . . . . . . . . 36

3.2.3. Crystallization of DCTN1 $1_{412-533}-\mathrm{IC}_{2-84}$ construct . . . . . . . . 37

3.2.4. Crystallization of DCTN1 $1_{412-533}-\mathrm{IC}_{2-69}$ construct . . . . . . . . . 37

3.3. Data processing . . . . . . . . . . . . . . . . . . . . . . . 37

3.4. Phase solution with selenomethionine . . . . . . . . . . . . . . . . 38

3.5. Model building . . . . . . . . . . . . . . . . . . . . . . . . . . . . 41

3.5.1. Molecular replacement with DCTN1 ${ }_{412-533}-\mathrm{IC}_{2-47}$ dataset taken from crystal grown at $\mathrm{pH} 7.3$. . . . . . . . . . . . . . . . . 43

3.5.2. Extending phases to new native dataset . . . . . . . . . . 43

3.6. Overview of the structural characteristics of the DCTN1 $1_{412-533}$ coiled-coil 45

3.7. Characteristics of dynein intermediate chain . . . . . . . . . . . . 52

3.8. Chemical cross-linking of DCTN1 ${ }_{412-533}-\mathrm{IC}_{2-47}$ and $\mathrm{DCTN1}_{412-533}-\mathrm{IC}_{2-69} \quad 56$

4. Discussion 61

4.1. Constructs DCTN1 $1_{412-533}-\mathrm{IC}_{2-47}$ and DCTN1 ${ }_{412-533}-\mathrm{IC}_{2-69}$ produced crystals . . . . . . . . . . . . . . . . . 6 6 61

4.2. Crystallization condition could be responsible for missing IC in crystals 63

4.3. DCTN1 $_{412-533}$ forms a coiled-coil . . . . . . . . . . . . . . . . . . 63

4.4. N-terminal IC is more likely to be a single $\alpha$-helix than a coiled-coil . . 65

4.5. Possible dynein-dynactin interaction models . . . . . . . . . . . . . . 66 
\begin{tabular}{lr}
\hline Bibliography & 69
\end{tabular}

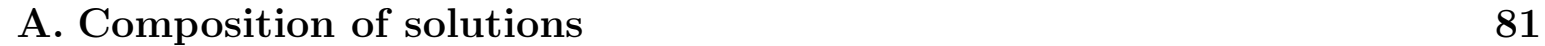

A.1. SDS-PAGE . . . . . . . . . . . . . . . . . . . . . 81

A.2. SDS sample buffer . . . . . . . . . . . . . . . . . . 82

A.3. Coomassie staining . . . . . . . . . . . . . . . . . . . 82

A.4. Determining protein concentration with Bradford . . . . . . . . . . . . 83

B. Plasmid map $\quad 85$

\begin{tabular}{ll}
\hline C. Additional result files & 87
\end{tabular}

C.1. Helical wheel representation of DCTN1 ${ }_{412-533}$. . . . . . . . . . . . 87

C.2. Example fragmentation spectra from cross-linking experiments . . . . . 88 



\begin{tabular}{|c|c|}
\hline$\AA$ & Ångström \\
\hline aa & amino acids \\
\hline AAA & $\underline{\text { ATPases }}$ associated with diverse cellular activities \\
\hline $\mathrm{ABC}$ & ammonium bicarbonate \\
\hline $\mathrm{ACMI}$ & Automatic Crystallographic Map Interpretation (program suite) \\
\hline $\mathrm{ACN}$ & acetonitrile \\
\hline $\operatorname{Arg}$ & arginine \\
\hline Arp & actin-related protein \\
\hline Asp & aspartic acid \\
\hline ATP & adenosine triphosphate \\
\hline bp & base pairs \\
\hline $\mathrm{BS}^{3}$ & bis[sulfosuccinimidyl] suberate \\
\hline BSA & bovine serum albumin \\
\hline CapZ & actin-capping protein \\
\hline $\mathrm{CC}_{1 / 2}$ & correlation coefficient \\
\hline CNS & crystallography \& NMR system \\
\hline CSAH & charged single $\alpha$-helix \\
\hline $\mathrm{cv}$ & column volume \\
\hline D. melanogaster & Drosophila melanogaster \\
\hline D. discoideum & Dictyostelium discoideum \\
\hline Da & Dalton \\
\hline DHC & dynein heavy chain \\
\hline DIC & dynein intermediate chain \\
\hline DNA & deoxyribonucleic acid \\
\hline DTT & 1,2-dithiole-3-thione \\
\hline E. coli & Escherichia coli \\
\hline EDTA & ethylene diamine tetraacetic acid \\
\hline EGTA & ethylene glycol tetraacetic acid \\
\hline Glu & glutamic acid \\
\hline $\mathrm{HC}$ & heavy chain \\
\hline Hepes & 4-(2-hydroxyethyl)-1-piperazineethanesulfonic acid \\
\hline His & histidine \\
\hline IC & intermediate chain \\
\hline IFT & intraflagellar transport \\
\hline IPTG & isopropyl $\beta$-D-1-thiogalactopyranoside \\
\hline $\mathrm{kb}$ & kilo base \\
\hline LB & Luria-Bertani medium \\
\hline
\end{tabular}




\begin{tabular}{|c|c|}
\hline $\mathrm{LC}$ & light chain \\
\hline LIC & light intermediate chain \\
\hline Lys & lysine \\
\hline $\mathrm{M}$ & molar \\
\hline MAD & multi-wavelength anomalous diffraction \\
\hline MCS & multiple cloning site \\
\hline MES & 2-(N-morpholino)ethanesulfonic acid \\
\hline MTBD & microtubule-binding domain \\
\hline MWCO & molecular weight cut-off \\
\hline NCBI & National Center for Biotechnology Information \\
\hline NCS & non-crystallographic symmetry \\
\hline $\mathrm{nm}$ & nanometre \\
\hline NTA & nitrilotriacetic acid \\
\hline NTP & nucleoside triphosphate \\
\hline $\mathrm{OD}_{600}$ & optical density for light of wavelength $600 \mathrm{~nm}$ \\
\hline PCR & polymerase chain reaction \\
\hline PEG & polyethylene glycol \\
\hline PMSF & phenylmethylsulfonyl fluoride \\
\hline $\mathrm{R}_{\text {work/free }}$ & residual factor / reliability factor \\
\hline $\operatorname{rcf}$ & relative centrifugal force \\
\hline r.m.s.d. & root-mean-square deviation \\
\hline rpm & rotations per minute \\
\hline $\mathrm{SAD}$ & single-wavelength anomalous diffraction \\
\hline SDS(-PAGE) & sodium dodecyl sulfate (polyacrylamide gel electrophoresis) \\
\hline SLS (Villigen) & Swiss Lightsource \\
\hline $\mathrm{T}_{\mathrm{M}}$ & melting temperature $\left[{ }^{\circ} \mathrm{C}\right]$ \\
\hline $\mathrm{U}$ & units \\
\hline TAE & Tris-acetic-EDTA buffer \\
\hline TEV & tobacco etch virus protease \\
\hline Thr & threonine \\
\hline Tris & tris(hydroxymethyl)aminomethane \\
\hline Tyr & tyrosine \\
\hline$\$ / X S C A L E / X D C O N V$ & program suite, see $($ Kabsch, 2010) \\
\hline
\end{tabular}




\section{Introduction}

\subsection{Motor proteins}

Eukaryotic cells are subject to a diverse range of biological processes including transport of vesicles, re-localization of organelles and cell division. An active and directed movement is crucial for these processes. Therefore, cells possess a diverse set of motor proteins, which move along cytoskeletal tracks.

These motor proteins are represented by the superfamilies of dynein, kinesin, and myosin. In order to generate motion they hydrolyse ATP in their motor domain. The conversion of chemical energy into mechanical movement is governed by conformational changes in the motor domain. This results in the consecutive attachment and detachment of the molecular motor from the cytoskeleton leading to directional motion along the tracks (Schliwa and Woehlke, 2003).

The short-range transport along actin filaments is executed by the myosin family, which mainly move towards the fast growing plus-end (Hartman and Spudich, 2012). Dyneins and kinesins on the other hand are necessary for the long-range transport using microtubules. Kinesins are mainly plus-end directed motor proteins moving cargo from the centre to the cell periphery, which is called anterograde transport (Hirokawa et al., 2009). Dynein moves exclusively to the minus-end of microtubules. It is responsible for the retrograde transport from the cell periphery back to the cell centre (Roberts et al., 2013).

While kinesins and myosins share a common ancestor (Kull et al., 1998) dyneins evolved from the $\mathrm{AAA}^{+}$superfamily $(\mathrm{AAA}=$ ATPases associated with diverse cellular activities) (Neuwald et al. 1999). Furthermore, dynein is characterised by a higher molecular complexity. The dynein heavy chain (DHC) alone is twice the size of muscle myosin and even four times the size of conventional kinesin Asai and Koonce, 2001). Another interesting aspect is the specialization of the different kinesins (15 classes (Hirokawa et al. 2009) ) and myosins (35 classes (Odronitz and Kollmar, 2007)) for certain functions in the cytoplasm, which is not found to that extent for dyneins. For example, myosin Va seems to be necessary for the transport of peripheral endoplasmatic reticulum into the dendritic spines of special neuronal cells whereas myosin 
XIX is needed for the transport of mitochondria in human cells (Hartman and Spudich, 2012). Examples for kinesin include kinesin-3 motors, which among others transport synaptic vesicle precursors and together with kinesin-1 motors are involved in the mitochondria transport (Hirokawa et al., 2009). How dynein manages to adapt to all its different functions in the cell will be explained hereafter.

\subsection{Dynein}

Dynein was first discovered about 50 years ago as an ATPase in cilia (Gibbons and Rowe, 1965). It was named after the unit of force, dyne.

The dynein family consists of nine major heavy chain classes, which can be grouped into two branches, the cytoplasmic and the axonemal dyneins. Cytoplasmic dynein 1 is expressed ubiquitously and is necessary for almost every minus-end directed microtubule transport in the cell (Kardon and Vale, 2009). Cytoplasmic dynein 2 is important for assembly of cilia and flagella (Pazour et al., 1999) and the intraflagellar transport (IFT) in the axoneme towards the cell body. The other seven dyneins are anchored within the axoneme where they are responsible for the coordinated beating of cilia and flagella to cause motion (Wickstead and Gull, 2007).

\subsubsection{Structure of cytoplasmic dynein}

Cytoplasmic dynein 1 is often referred to as cytoplasmic dynein. It is a large $\approx 1.5 \mathrm{MDa}$ multi-subunit complex consisting of two homodimeric heavy chains (DYNC1H1) each $\approx 500 \mathrm{kDa}$ as well as various additional subunits (see figure 1.1).

The dynein heavy chain (DHC) is made up of the $10 \mathrm{~nm}$ long stalk, followed by a ring of six $\mathrm{AAA}^{+}$domains, which is attached to the N-terminal linker and the tail domain. The latter is responsible for the homodimerization of the two heavy chains and acts as a scaffold for the assembly of dynein subunits, which bind as dimers. The six $\mathrm{AAA}^{+}$

domains are encoded by a single polypeptide, which is unusual for ATPases (Carter et al., 2011). Only the first four $\mathrm{AAA}^{+}$domains (AAA1 to AAA4) contain functional ATP binding sites. However, only the hydrolysis of ATP in AAA1 is essential for the movement along microtubules. AAA2-AAA4 can also hydrolyse/bind ATP though they only seem to influence motility (Kon et al., 2004). From the AAA4 domain the stalk emerges with the microtubule-binding domain (MTBD) at its tip. This stalk is supported at the base by a buttress (Carter et al., 2011) or strut (Kon et al., 2011) 


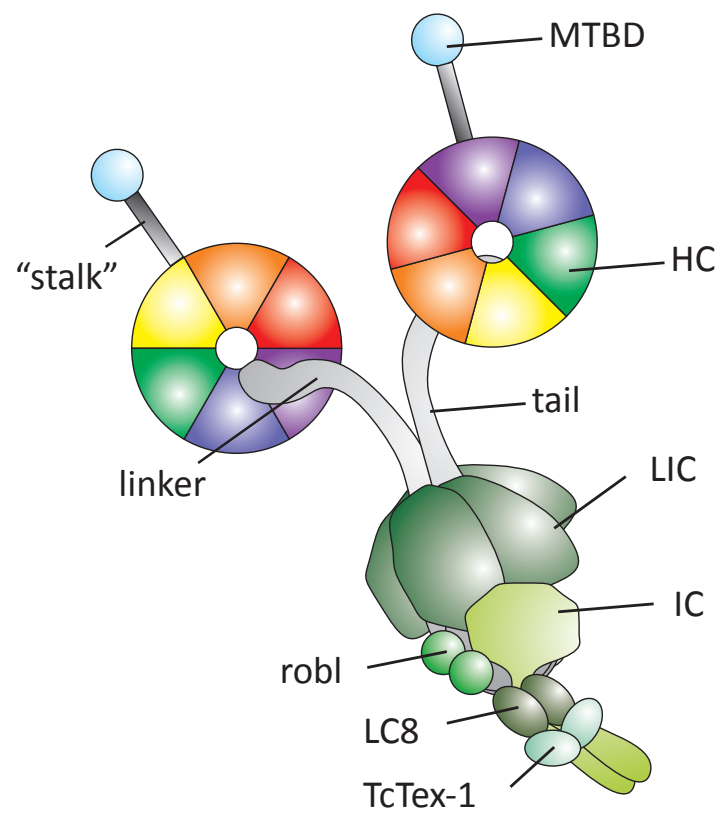

Figure 1.1.: Schematic overview of cytoplasmic dynein. The representation shows the possible composition of subunits. HC - Heavy chain, IC - Intermediate chain, LC8 - Light chain 8, LIC - Light intermediate chain, MTBD - microtubule-binding domain, robl - Roadblock light chain, TcTex-1 - Light chain (T-complex testis-specific protein 1)

extending out from the AAA5 domain. It may play a role in transferring the ATP state of the hexameric $\mathrm{AAA}^{+}$ring to the MTBD (Carter, 2013).

The linker arches over the surface of the $\mathrm{AAA}^{+}$ring interacting with AAA5 and AAA1 and goes down next to the AAA1 domain (Carter et al., 2011). It is thought to deliver the powerstroke (Roberts et al., 2013).

Out of the remaining dynein subunits only two intermediate chains (IC; $\approx 74 \mathrm{kDa}$ ) and four light intermediate chains (LIC; $50-60 \mathrm{kDa}$ ) assemble directly on the N-terminus of the DHC (Tynan et al., 2000). The smaller light chains (LC) light chain 8 (LC8; $\approx 8$ $\mathrm{kDa}$ ), roadblock light chain ( $\mathrm{robl} ; \approx 10 \mathrm{kDa}$ ), and T-complex testis-specific protein 1 (TcTex-1; $\approx 14 \mathrm{kDa}$ ) bind as dimers to the N-terminus of the intermediate chain dimer (see figure 1.2) (Hall et al., 2009, 2010, Makokha et al., 2002, Susalka et al., 2002). While IC and LIC are only associated with dynein the LCs can be found in connection with other binding partners (Pfister et al., 2006).

The dimeric roadblock light chain is structurally unrelated to LC8 and TcTex-1. It is bound by two IC $\alpha$-helices about 100 residues away from the other two LCs (Hall et al. 2010). LC8 and TcTex-1 are homologous proteins with similar IC binding sites, which lie close to each other. While unbound IC is disordered (Benison et al., 2006) the binding of LC8 and TcTex-1 leads to the formation of extended $\beta$-strands of the 
recognition sites (Hall et al., 2009). Moreover, the binding of one LC is enhanced when another LC is already bound to IC (Williams et al., 2007). Hence it is assumed that these light chains have a polybivalent scaffold, which enhances the affinity of every bivalent binding partner like the binding of LCs and coiled-coil interactions (Hall et al., 2009). This assumption is supported by the finding that the binding efficiency of an adaptor protein (dynactin DCTN1) to IC containing four bivalent sites is higher in contrast to IC with only one site (King et al., 2003). Both light chains were known to have various binding partners and it was presumed that they act as adapters for cargo binding for the dynein transport. However, recent studies showed the cargo-binding sites of the IC bound LC8 homodimer are occupied by IC making it impossible to bind cargo at the same time (Hall et al., 2009). Therefore, it has been suggested that LC8 rather acts as a hub protein promoting the activation and dimerization of its binding partners, like IC (Barbar, 2008). Besides the interaction sites of LCs, the N-terminus of IC contains interaction motifs for various other proteins that are necessary for the essential functions of dynein. Amongst others IC binds to the dynactin subunit DCTN1, huntingtin, the nuclear distribution protein E (NudE), and ZW10 (Kardon and Vale, 2009).

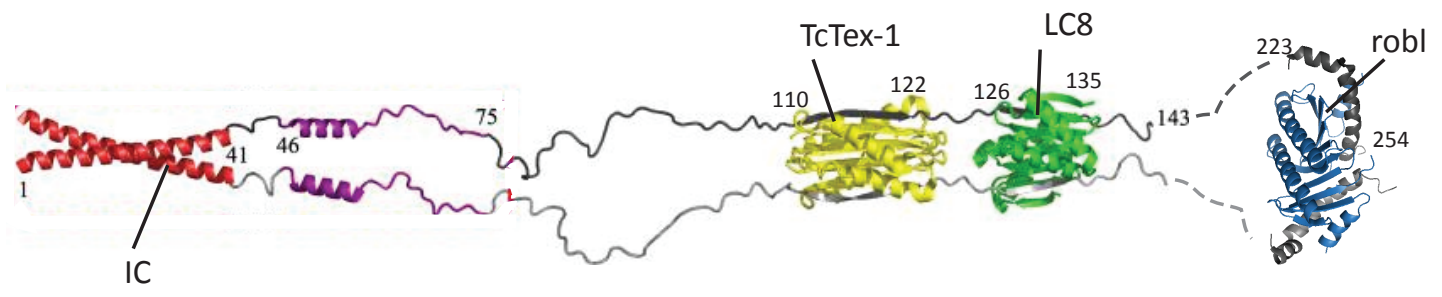

Figure 1.2.: Model of the assembly of dynein intermediate chain and its light chains. The model is a modified image based on the work of Morgan et al. (2011) and Hall et al. (2010). The structures are derived from NMR and X-ray experiments using recombinantly expressed $D$. melanogaster proteins. Numbers near the bound light chains indicate the amino acids positions of IC that interact with the respective light chains. The N-terminal numbers denote IC regions that are necessary for binding to other proteins. grey: IC - Intermediate chain, red/purple: regions of IC supposedly interacting with dynactin, blue: robl - Roadblock light chain, green: LC8 - Light chain 8, yellow: TcTex-1 (T-complex testis-specific protein 1)

Furthermore, it could be shown that mammals have two IC proteins (encoded by Dync1i1 and Dync1i2). Analysis of mouse IC showed that they undergo alternative splicing at several exons coding for the N-terminal region of the proteins. The expression of the various isoforms is tissue specific. Most spliced isoforms of both IC proteins were found in brain-specific tissues which may reflect the complexity of the cargoes transported in neurons (Kuta et al., 2010). 


\subsubsection{Functions of cytoplasmic dynein}

Cytoplasmic dynein is necessary for numerous activities in the cell. It plays a major role in microtubule organization during mitosis. It could be demonstrated that injection of antibodies against dynein $\mathrm{HC}$ during mitosis led to defects in spindle morphology (Vaisberg et al., 1993) and chromosomes were unaligned when the dyneinactivating protein, dynactin, was disrupted (Echeverri et al., 1996). Together with other proteins dynein can also be found attached to kinetochores of chromosomes during prometaphase. There, it is necessary for capturing microtubules and powering the movement of chromosomes towards spindle poles before they align on the metaphase plate (Yang et al., 2007). Additionally, dynein delays anaphase until all chromosomes are attached to the spindle (Foley and Kapoor, 2013). Then it removes the spindleassembly checkpoint proteins by transporting them towards the spindle poles, which leads to the separation of chromosomes during anaphase (Howell et al., 2001).

Dyneins can also be found attached to the cell cortex applying a pulling force to microtubules by either moving towards the minus-end or binding to a disassembling plus-end (Hendricks et al. 2012). This leads to a stabilization of microtubule plus-ends and regulates microtubule dynamics.

Furthermore, dynein is involved in the organisation and localisation of the Golgi as well as in the minus-end directed transport of endosomes, lysosomes, and lipids to the cell centre. It also plays a role in the vesicle transport from endoplasmatic reticulum to the Golgi. Experiments could demonstrate that the disruption of dynein using specific antibodies lead to the fragmentation of the Golgi (Vaisberg et al., 1996). A disruption of dynein heavy chain in mice caused a re-localization of lysosomes and endosomes as well as an inhibition of the vesicle transport from the endoplasmatic reticulum to the Golgi (Burkhardt et al., 1997, Harada et al., 1998, Presley et al., 1997).

\subsection{The activator of dynein: dynactin}

In order to achieve all the above mentioned functions dynein interacts with various adaptors and regulators. One of them is dynactin, which is necessary for almost every dynein process (Karki and Holzbaur, 1999, Schroer, 2004). Like dynein dynactin is a large multi-subunit complex $(\approx 1.1 \mathrm{MDa})$. It is a very ancient protein complex that can be found in every eukaryotic organism except for plants and diplomonads. The absence of dynactin in some species coincides with that of the dynein heavy chain. However, there are some species like Piroplasmida, which encode a divergent and shortened dynein heavy chain without possessing genes for dynactin. It is not known if these 
dynein motors are functional but it shows that the presence of dynactin is coupled to dynein (Hammesfahr and Kollmar, 2012).

Dynactin was first discovered in 1991 as a cytosolic activity that supported dynein to move vesicles over a long range along microtubules. Later that same year it could be attributed to the dynactin complex (Gill et al., 1991, Schroer and Sheetz, 1991). Electron microscopy analysis revealed two major segments, a helical filament about 10 $\times 37 \mathrm{~nm}$ and a projecting arm about $24 \mathrm{~nm}$ in length (Schafer et al., 1994a). The complex consists of 11 subunits of various sizes in unequal stoichiometry as shown in figure 1.3 (Schroer, 2004).

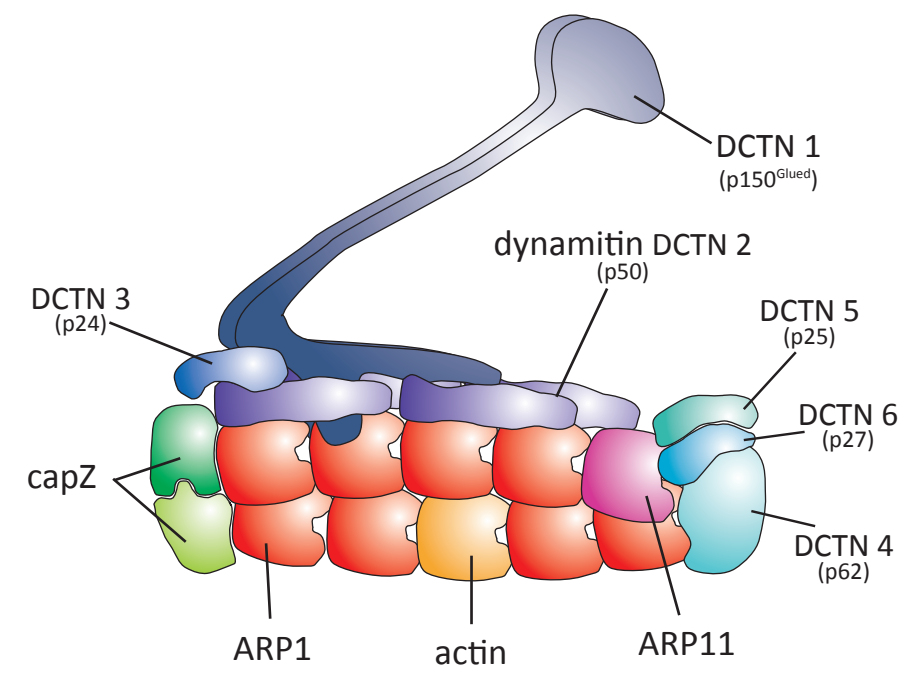

Figure 1.3.: Schematic overview of cytoplasmic dynein activator dynactin. The complex can be split in two parts, the rigid helical filament and the flexible arm-shoulder region. The helical filament is comprised of eight actinlike proteins 1 (Arp1) and one conventional actin. On the barbed-end it is caped by the capping protein CapZ. On the opposite site it is terminated by the pointed-end complex comprised of Arp11, DCTN4, DCTN6, and DCTN5. The protruding arm consists of a DCTN1 dimer, which interacts with four DCTN2 and two DCTN3 molecules.

The helical structure is a mini actin-filament, which is comprised of actin-related proteins Arp1 $(8 \times)$ and Arp11 $(1 \times)$, as well as a conventional actin molecule. Just like conventional actin, Arp1 is capable of hydrolysing ATP and forming filaments (Bingham and Schroer, 1999). But unlike actin, Arp1 forms very stable, short filaments of identical length. It is able to bind to organelle-associated $\beta$-spectrin III. Therefore, it is hypothesized to recruit dynein to intracellular membranes for further transport (Holleran et al., 2001).

The filament is capped on one side with the typical actin-capping protein, CapZ, suggesting an Arp1 structure similar to the barbed (plus) end of actin (Schafer et al., 
1994a b). It consists of two subunits (Cap32 and Cap34), which despite low sequence homology exhibit very similar secondary structures (Eckert et al. 2012). On the opposing end sits the heterotetrameric pointed-end complex consisting of Arp11, DCTN4, DCTN5, and DCTN6 (Eckley et al., 1999, Schafer et al., 1994a). Due to the actin-like sequence of Arp11 this subunit can bind to Arp1, but denies further attachment of Arp1 molecules. This suggests a capping function for Arp11.

The DCTN4 sequence predicts a zinc-binding RING or LIM motive close to the Nterminus (Eckley et al., 1999, Karki et al., 2000). These motifs generally participate in protein-protein interactions. Deletion of the zinc-binding motif did not disrupt the interaction with ARP1 or actin suggesting a role in binding to other dynactin subunits or to other subcellular structures (Garces et al., 1999, Vaughan et al., 1999). However, the absence of DCTN4 does not significantly affect dynactin stability (Lee et al., 2001). Furthermore, DCTN4 contains a highly conserved C-terminal metal-binding domain (CXXC), which could play a role in the copper-dependent interaction with an ATPase, ATP7B. This ATPase is important for maintaining the copper homeostasis in liver cells. The transport of ATP7B to vesicles of liver cells is necessary for the removal of excess copper into the bile (Lim et al., 2006).

The two remaining pointed-end complex proteins, DCTN5 and DCTN6, are predicted to adopt an unusual left-handed $\beta$-helical fold leading to the formation of a small triangular prism (Parisi et al., 2004). The recently solved structure of DCTN6 could confirm this prediction (Yeh et al., 2013). Due to the similar prediction for DCTN5 a hydrophobic interaction between both proteins based on the DCTN6 structure is proposed. Furthermore, it could be shown that mitotic phosphorylation of DCTN6 during prometaphase is required for targeting polo-like kinase 1 to kinetochores. This in turn is important for the attachment of microtubules to the kinetochore (Yeh et al., 2013). The DCTN5 subunit is required for the dynein-mediated early endosome movement. Its loss caused early endosome accumulation (Zhang et al., 2011).

The flexible structure protruding from the barbed-end of the Arp1-filament consists of the three subunits DCTN1, DCTN2 (dynamitin), and DCTN3. DCTN1 is the largest of all dynactin subunits $(\approx 150 \mathrm{kDa})$. Each complex features a homodimer consisting of two DCTN1 molecules, which are predicted to exhibit two coiled-coils ( $\approx 50$ and $20 \mathrm{~nm}$ in length) interrupted by regions of undefined structure Gill et al., 1991, Holzbaur et al., 1991). On the extreme end of the N-terminus is a small globular head with a CAP-Gly motif (cytoskeleton-associated protein, glycine-rich) that can bind directly to microtubules in a nucleotide-independent manner (Waterman-Storer et al., 1995). Together with another microtubule-binding protein, EB1, the CAP-Gly domain forms a complex to stabilize microtubule plus-ends (Ligon et al., 2003). Next to the CAP- 
Gly domain is a basic domain, which could be shown to increase the processivity of cytoplasmic dynein (Culver-Hanlon et al., 2006). This domain is followed by the first, $\approx 50 \mathrm{~nm}$ long coiled-coil. Since the flexible arm protrudes $\approx 24 \mathrm{~nm}$ out of the helical filament it is assumed that half of the coiled-coil is contained within the shoulder region (Schafer et al., 1994a, Schroer, 2004). Furthermore, the first DCTN1 coiled-coil domain provides a platform for the binding of several microtubule-based motor proteins. Here, not only dynein interacts with its activator but also kinesin II (Deacon et al., 2003) and the kinesin-related motor Eg5 (Blangy et al., 1997). This demonstrates that dynactin is necessary for the retrograde as well as anterograde transport. The second coiled-coil domain is assumed to be important for the binding to the Arp1-filament since it contains a conserved actin-binding motif (Waterman-Storer et al., 1995). This is supported by the finding that DCTN1 lacking the C-terminus is not incorporated into the dynactin complex (McGrail et al., 1995). A further essential part of DCTN1 binding to the helical filament is DCTN2. It forms elongated tetramers and probably self-associates via coiled-coils (Echeverri et al., 1996). Overexpression of this molecule leads to the disassembly of DCTN1 and DCTN3 from the remaining dynactin complex (Echeverri et al., 1996) (Karki et al., 1998) showing that DCTN2 is necessary for DCTN1-binding. The exact binding mechanism is unknown but it is speculated that two C-terminal coiled-coil regions interact with DCTN1 and DCTN3. Furthermore, DCTN3 plays an essential role in the formation of this subcomplex as it interacts with DCTN1 and DCTN2 (Terasawa et al., 2010).

\subsection{Aim of this work}

At present the knowledge about the dynactin complex on an atomic level is limited. X-ray structures exist for the CAP-Gly domain of DCTN1 (Honnappa et al., 2006), actin (Otterbein et al., 2001), the subcomplex of the barbed-end capping protein CapZ (Eckert et al., 2012), and DCTN6 (Yeh et al., 2013). Little else is known about the other subunit structures and their interactions amongst each other.

In this study the dynein-dynactin interaction site is analysed by applying protein crystallography and chemical cross-linking. Since the discovery of the dynactin complex several studies have worked on elucidating the binding site. It could be shown that the extreme N-terminus of the dynein intermediate chain (IC) and the first coiled-coil of the dynactin subunit DCTN1 are involved in the interaction (Morgan et al., 2011, Siglin et al., 2013, Vaughan and Vallee, 1995). The interaction sites for both proteins have been narrowed down by different approaches. NMR experiments could determine two binding regions in the N-terminal region of IC of Drosophila melanogaster. 
It consists of a helical section between amino acids 1-41 and a nascent helix comprised of residues 46-75 (Morgan et al., 2011). The Ph.D. thesis of A. Siglin (2010) reported a protein gel-shift experiment with recombinantly expressed rat DCTN1-and IC-fragments of various sizes to identify the minimum sizes of both proteins to form a complex. It could show that the first 44 amino-acids of IC and amino-acids 415-530 in the coiled-coil 1 region of DCTN1 are sufficient to form a complex. However, it is still not understood how they interact on the molecular level. 



\section{Materials and methods}

\subsection{Chemicals and equipment}

Chemicals for the preparation of solutions, buffers, and media were purchased from the companies Carl Roth GmbH \& Co. KG (Karlsruhe, D), Sigma-Aldrich Chemie GmbH (Steinheim, D), and Merck Millipore (Darmstadt, D). Restriction enzymes and their suitable buffers were acquired from Thermo Fisher Scientific Fermentas (Vilnius, LIT) and New England Biolabs (Ipswich, US-MA). For the preparation of plasmid-DNA from Escherichia coli, the extraction of DNA from agarose gels, and PCR purification, the kits from the company MACHEREY-NAGEL (Düren, D) were used.

\subsection{Strains, plasmids and oligonucleotides}

Escherichia coli XL-2 blue (endA1 supE44 thi-1 recA1 gyr A96 relA1 lac) was used for cloning while $E$. coli BL21-CodonPlus(DE3)RIL $\left(F^{-}\right.$ompT $h s d S\left(r_{B}^{-} m_{B}^{-}\right) d c m^{+}$ Tet ${ }^{r}$ gal $\lambda(\mathrm{DE} 3)$ endA Hte $\left[\right.$ argU ileY leuW $\left.\left.\mathrm{Cam}^{r}\right]\right)$ was used for expressing the target proteins.

For the expression of selenomethionine containing proteins methionine auxotroph $E$. coli 834 cells ( $h s d S$ metE gal ompT) were applied.

Oligonucleotides were synthesized by Eurofins MWG Operon (Ebersberg, D) and can be found in table 2.1. 
Table 2.1.: List of oligonucleotides

\begin{tabular}{|c|c|c|c|}
\hline Primer & Nucleotide sequence $5^{\prime} \rightarrow 3^{\prime}$ & $\begin{array}{l}\text { Restriction } \\
\text { Site }\end{array}$ & $\mathbf{T}_{\mathbf{M}}\left[{ }^{\circ} \mathbf{C}\right]$ \\
\hline PF1 & ATACCATGGGCAGCCAAG TTCAACCAAAATATTTTGAA AAATCTCAA & $N c o \mathrm{I}$ & 51.7 \\
\hline $\mathrm{PF} 2 \mathrm{~b}$ & $\begin{array}{l}\text { CCGGGATCCGTCTCAATA TTATACAATTATTACCAATA } \\
\text { TAAACATTTGA }\end{array}$ & BamHI & 48.0 \\
\hline PF7 & ATACCATGGGCAGCTCTG ACAAAAGTGACTTAAAAGCTGAG & $N c o I$ & 52.9 \\
\hline $\mathrm{PF} 8 \mathrm{~b}$ & CGCAAGCTTTTACT GAACGGGTTCTTTCTTCTGC & HindIII & 54.1 \\
\hline PF9 & GGTTATGTACAAGGTGCGG AGAAAACGGTCGATGAGGTC & $B s r \mathrm{GI}$ & 58.8 \\
\hline PF10b & GAGACGCGTTTACGCCT CCTGCTGACTCATGAGC & MluI & 58.7 \\
\hline PF8-2 & CGCAAGCTTTTATTT CGAGGAGGGAGACATAGG & HindIII & 52.8 \\
\hline PF7-His-TEV & $\begin{array}{l}\text { CAGGATCCGGGTGAAAACC TGTATTTTCAGGGCTCT } \\
\text { GACAAAAGTGACTTAAAAGCTGAG }\end{array}$ & $\begin{array}{l}\text { BamHI } \\
\mathrm{TEV}\end{array}$ & 52.9 \\
\hline PF9-ohneHis & ATACATATGGCGGAGAA AACGGTCGATGAGCTG & NdeI & 58.8 \\
\hline PF8-3 & CGCAAGCTTTTATG AGATACCAATGCTTTGCAGCA & HindIII & 51.6 \\
\hline
\end{tabular}


Table 2.2.: List of plasmids

\begin{tabular}{|c|c|c|}
\hline Plasmid & Description & Source \\
\hline pRSFmD1mako & $\begin{array}{l}3815 \text { bp; expression vector for Escherichia coli designed from pRSFDuet-1 } \\
\text { (NovAGEN), contains two multiple cloning sites (MCS) for the coexpression of } \\
\text { two target genes, each MCS contains a N-terminal } 6 \times \text { His-Tag (Smith et al. } \\
\text { 1988) and a T7-promoter; RSF origin, kanamycine resistance cassette for se- } \\
\text { lection (see B.1. }\end{array}$ & PD Dr. Martin Kollmar \\
\hline \multicolumn{3}{|c|}{ Constructs based on aforementioned vector } \\
\hline pMK186 & human dynein intermediate chain (DIC)(aa 1-701) in pDXAmako2b & Maria Faberova \\
\hline pMK146b & chicken DCTN1 coiled-coil 1 (aa 209-540) in pDXAmako4 & Martin Kollmar \\
\hline pMK220 & $\begin{array}{l}\text { human DIC (aa } 2-47 \text { ) and chicken dynactin DCTN1 (aa } 412-533 \text { ) with } \\
\text { N-terminal } 6 \times \text { His-tag cloned into pRSFmD1mako }\end{array}$ & this study \\
\hline pMK242 & $\begin{array}{l}\text { human DIC (aa } 2 \text { - } 87 \text { ) and chicken DCTN1 (aa } 412 \text { - 533) with N-terminal } \\
6 \times \text { His-tag cloned into pRSFmD1mako }\end{array}$ & this study \\
\hline pMK243 & $\begin{array}{l}\text { human DIC (aa } 2 \text { - } 47 \text { ) with N-terminal } 6 \times \text { His-Tag and TEV restriction site } \\
\text { and chicken DCTN1 (aa } 412-533 \text { ) cloned into pRSFmD1mako }\end{array}$ & this study \\
\hline pMK248 & $\begin{array}{l}\text { human DIC (aa 2-69) and chicken DCTN1 (aa } 412 \text { - 533) with N-terminal 6× } \\
\text { His-tag cloned into pRSFmD1mako }\end{array}$ & this study \\
\hline
\end{tabular}




\subsection{Cloning techniques}

\subsubsection{Polymerase chain reaction $(\mathrm{PCR})$}

Genes of interest were amplified via polymerase chain reaction (Saiki et al., 1988). For the amplification the Expand High Fidelity PCR System from Roche APPLIED SCIENCE (Penzberg, D) was used. Reactions were set up with $10 \times$ PCR buffer supplied by the company, $2.75 \mathrm{U}$ of the enzyme mix (containing Taq and Tgo DNA polymerase), $0.25 \mathrm{mM}$ of each dNTP, $100 \mu \mathrm{M}$ of each primer, 2 - $20 \mathrm{ng}$ of template DNA, and water to add up to a total volume of $50 \mu \mathrm{l}$.

PCR reactions were carried out in a PCR Sprint thermal cycler from HYBAID (Ashford, UK). 20 cycles of denaturation of double-stranded DNA (1 min, $\left.94{ }^{\circ} \mathrm{C}\right)$, annealing of primers to single-stranded DNA (1 min, varying temperature $\left.{ }^{1}\right)$ followed by an elongation step $\left(1 \mathrm{~min}\right.$ at $\left.72{ }^{\circ} \mathrm{C}\right)$ were carried out ${ }^{2}$. PCR products were checked by applying $5 \mu \mathrm{l}$ of the reaction sample on a $1 \%$ agarose gel.

\subsubsection{PCR purification}

PCR products were purified from primers, nucleotides, polymerases, and salts with the NucleoSpin Gel and PCR Clean-up kit (MaCherey-NAGEL). The company's manual was followed.

\subsubsection{Restriction digestion of DNA}

Plasmids and PCR products were digested for analytical and preparative purposes. Restriction digests (Nath and Azzolina, 1981) were carried out with restriction endonucleases from Fermentas or New England Biolabs and their suitable buffers for 1 hour at $37^{\circ} \mathrm{C}$. The products were analysed on a $1 \%$ agarose gel and when necessary cut out for further cloning (see subsection 2.3.5).

\subsubsection{Agarose gel electrophoresis}

Prior to gel electrophoresis (Sharp et al., 1973) 6× DNA loading dye (40\% Ficoll 400, $0.5 \%$ bromphenol blue, $0.5 \%$ xylene cyanol) was added to the DNA samples. The samples were separated together with a DNA marker (GeneRuler 1 kb DNA Ladder, Thermo Fisher SCIENTIFIC) according to size in a horizontal agarose gel containing $1 \%$ agarose in TAE buffer (40 mM Tris-Cl, $19 \mathrm{mM}$ glacial acetic acid, $1 \mathrm{mM}$ EDTA

\footnotetext{
${ }^{1}$ Annealing temperature equals melting temperature of primer minus 5 to $10{ }^{\circ} \mathrm{C}$.

${ }^{2}$ Elongation time depends on fragment length. In $1 \mathrm{~min}$ up to $1.5 \mathrm{~kb}$ can be amplified, which was sufficient for all fragments.
} 
$\mathrm{pH} 8.0)$ and $\sim 0.005 \%$ ethidium bromide, which intercalates into the DNA and fluoresces under UV-light. The gel was run in TAE buffer at $100 \mathrm{~V}$ for $60 \mathrm{~min}$ and then the DNA samples were visualized with the UV transilluminator TFX-20M (LIFE Technologies, Carlsbad, CA-US).

\subsubsection{Gel extraction of DNA fragments}

Digested DNA was separated on $1 \%$ agarose gels. The desired DNA fragments were excised from the agarose gels with a clean scalpel for subsequent ligation. After that, DNA was extracted from the gels with the NucleoSpin ${ }^{\circledR}$ Gel and PCR Clean-up kit (MACHEREY-NAGEL) following the company's manual.

\subsubsection{Ligation of DNA fragments}

For the ligation (Sugino et al., 1977) reaction vector and gel extracted DNA fragments were mixed in a ratio of 1:3 or 3:5 in a total volume of $10 \mu \mathrm{l}$. The incubation took place in $10 \times$ ligation buffer and $5 \mathrm{U}$ T4 ligase (FERMENTAS) for two hours at room temperature $\left(\sim 20^{\circ} \mathrm{C}\right)$. The reaction sample was then mixed with $50 \mu \mathrm{l} E$. coli XL-2 blue.

\subsubsection{Plasmid purification}

Plasmid purification was performed on colonies derived from ligation and subsequent transformation into E. coli XL-2 blue. A colony was picked from a LB plate and inoculated in $3 \mathrm{ml}$ of LB in a culture tube with the appropriate antibiotics and incubated at $37^{\circ} \mathrm{C}$, shaking overnight. Then, $1 \mathrm{ml}$ of the culture was centrifuged at 11,000 rcf for 30 seconds. The resulting pellet was used for extracting the plasmid with the NucleoSpin ${ }^{\circledR}$ Plasmid kit from MACHEREY-NAGEL following the company's manual. For the storage of positive plasmid constructs a midi prep with the NucleoBond ${ }^{\circledR}$ Xtra Midi kit was performed. With this method a higher yield of plasmid DNA can be achieved. 50 to $100 \mathrm{ml}$ LB medium with appropriate antibiotics was inoculated with a $E$. coli XL-2 blue colony derived from a transformation with positive plasmid DNA. The culture was incubated overnight at $37^{\circ} \mathrm{C}$, harvested and then the company's manual was followed to purify the plasmid.

\subsubsection{DNA sequencing}

For the verification of cloned DNA sequences the plasmids were sent to EUROFINS MWG Operon in Ebersberg. Following the suggested sample preparation, $15 \mu \mathrm{l}$ of 
50-100 ng/ $\mu$ l of plasmid DNA was mixed with $2 \mu \mathrm{l}$ of primer with a concentration of $10 \mathrm{pmol} / \mu \mathrm{l}$. The results were checked with the alignment program BioEdit (Hall, 1999).

\subsection{Transformation and cultivation of Escherichia coli}

\subsubsection{Transformation via heatshock}

Chemically competent $E$. coli cells were either incubated with a finished ligation reaction or 0.5 to $2 \mu \mathrm{g}$ of plasmid DNA. The reaction sample was incubated on ice for $30 \mathrm{~min}$ and then a heat shock of $30 \mathrm{sec}$ at $42{ }^{\circ} \mathrm{C}$ was applied (Cohen and Chang, 1973). The sample was immediately put back one ice for a further 5 min before spreading it on a LB plate with the appropriate antibiotics. The plate was then incubated at $37^{\circ} \mathrm{C}$ overnight.

\subsubsection{Cultivation media}

E. coli was cultivated in Luria-Bertani-Medium (LB) (Bertani, 1951) which is described in table 2.3 containing the appropriate antibiotics. For plates, $2 \%$ agar was added.

Table 2.3.: LB-Medium

\begin{tabular}{|l|l|}
\hline Luria-Bertani-Medium (LB) \\
\hline $\mathrm{NaCl}$ & $1 \%$ \\
Yeast extract & $0.5 \%$ \\
Peptone & $1 \%$ \\
\hline
\end{tabular}

\subsubsection{Synthesis of selenomethionine-containing proteins}

To obtain selenomethionine-labelled protein (Hendrickson et al., 1990) the plasmid had to be transformed into a methionine auxotroph strain like E. coli 834 (DE3) (NovagEN, Darmstadt, D). Then, a colony was inoculated in $10 \mathrm{ml} \mathrm{LB}$ broth containing the relevant antibiotics at $37{ }^{\circ} \mathrm{C}$ overnight. The cells were harvested and washed three times with $10 \mathrm{ml}$ sterile water to wash out any remaining LB substances. The final pellet was re-suspended in $1 \mathrm{ml}$ sterile water and inoculated in $1 \mathrm{~L}$ pre-warmed SelenoMet medium (ATHEnAES, Baltimore, MD-US) supplemented with $50 \mu \mathrm{g} / \mathrm{ml}$ selenomethionine (Sigma-AlDRICH). Cells were grown at $30{ }^{\circ} \mathrm{C}$ overnight before the addition of IPTG to a final concentration of $0.5 \mathrm{mM}$ and growth was continued for a further $6 \mathrm{~h}$ 
prior to harvest (40 min, 6,000 g). Protein purification was carried out following the same protocol as used for the native protein (see section 2.6).

\subsection{Protein expression test}

Protein expression tests were conducted to check whether the chosen expression temperature, the concentration of IPTG, and if required additives like ethanol were sufficient to produce the target protein in the folded state in the supernatant. Usually, expression was first tested at $37^{\circ} \mathrm{C}$ and if necessary down to $20^{\circ} \mathrm{C}$. In the case of $E$. coli ArcticExpress, expression was tested at $12{ }^{\circ} \mathrm{C}$.

$50 \mathrm{ml} \mathrm{LB}$ with the required antibiotics was inoculated with an overnight preculture at 1:50 dilution and grown at $37^{\circ} \mathrm{C}$, shaking $(120$ - $180 \mathrm{rpm})$ until an $\mathrm{OD}_{600}$ of 0.5 to 0.8 was reached. Prior to the addition of IPTG, $5 \mathrm{ml}$ of the culture were removed and centrifuged at $6,000 \mathrm{~g}$ for $15 \mathrm{~min}$. The pellet was frozen at $-20{ }^{\circ} \mathrm{C}$. IPTG was added at a final concentration of 0.1 to $1 \mathrm{mM}$. After that, $5 \mathrm{ml}$-samples were taken at certain time points (usually before IPTG, and 1, 3, 5, and 16 hours after IPTG induction), centrifuged and frozen as well.

To extract the protein, the collected samples were thawed on ice. Then $200 \mu$ lysis buffer (see table 2.4) were added to each sample, transferred into a $1.5 \mathrm{ml}$ Eppendorf tube and sonicated thrice for $10 \mathrm{sec}$ at $20 \%$ on ice with at least a $30 \mathrm{sec}$ break between each sonication step. Subsequently, the samples were centrifuged for $30 \mathrm{~min}$ at $16,000 \mathrm{~g}$ and $4{ }^{\circ} \mathrm{C}$. The supernatant was transferred into a new E-cup and $6 \times \mathrm{SDS}$ buffer (see table A.2) was added to both the supernatant and the pellet. Between 2 to $5 \mu \mathrm{l}$ of sample together with a protein marker (Bench-Mark ${ }^{\mathrm{TM}}$ Ladder INVITROGEN) were applied on a SDS-PAGE gel (see appendix A.1), run at $40 \mathrm{~mA}$ for $40 \mathrm{~min}$ and then stained with Coomassie blue (see appendix A.3.

\subsection{Protein purification}

$1 \mathrm{~L}$ of LB broth containing the appropriate antibiotics was inoculated with an overnight preculture at 1:50 dilution and allowed to grow at $37^{\circ} \mathrm{C}$ until an $\mathrm{OD}_{600}$ of $0.5-0.8$ was reached. Then, protein expression was induced by adding IPTG to a certain, previously tested final concentration. Cells were usually grown overnight, harvested by centrifugation at $6,000 \mathrm{~g}$ for $15 \mathrm{~min}$ at $4{ }^{\circ} \mathrm{C}$ and the resulting cell pellets were stored at $-20{ }^{\circ} \mathrm{C}$ for further experiments. 
The following protocol was the standard procedure for every newly tested protein. If the standard buffers proved to be unsuitable for the protein different buffers were tested. These changes are mentioned in the results chapter (see chapter 3).

\section{Sonication of pellets}

Previously frozen pellets were thawed on ice, re-suspended in ice-cold lysis buffer (see table 2.4 $15 \mathrm{ml}$ buffer per $4 \mathrm{~g}$ pellet) and disrupted on ice by sonication. A spatula tip of DNase (MP BiomedicAL) was added to the lysate and then centrifuged at 37,000 $\mathrm{g}$ for 40 min at $4{ }^{\circ} \mathrm{C}$ to remove cellular debris.

\section{Protein purification over Ni-NTA-column}

Beforehand, a column containing Ni-NTA superflow resin (QIAGEN, Hilden, D) to which proteins with His-tags can bind was pre-equilibrated with buffer A (see table 2.4). The supernatant was sterile filtered through a $0.22 \mu \mathrm{m}$ syringe filter (RoTH) then it was applied onto the column. The column was washed with 8 column volumes (cv) of buffer A. Then $10 \mathrm{cv}$ of buffer B followed by $8 \mathrm{cv}$ of buffer A containing $40 \mathrm{mM}$ imidazole $(\mathrm{pH} 7.3)$ were applied to remove non-specifically bound proteins from the column. Finally, the protein complex was eluted over 5-7 cv using a linear gradient of 40-500 $\mathrm{mM}$ imidazole in buffer A in $1 \mathrm{ml}$ fractions. Fractions from the peak area of the chromatogram were checked on SDS-PAGE gels.

\section{Dialysis}

Fractions containing the target protein were pooled, transferred into a dialysis membrane (MWCO 8-10 kDa, $25 \mathrm{~mm}$ ) (RoTh), and dialysed against $2 \mathrm{~L}$ buffer $\mathrm{C}$ (see table 2.4 at $4{ }^{\circ} \mathrm{C}$ overnight.

\section{Size exclusion chromatography}

After concentrating the protein with a Vivaspin concentrator (GE HEALTHCARE) appropriate for the size of the protein, the protein was further purified by size-exclusion chromatography on a HiLoad 16/60 Superdex 200 column (GE HEALTHCARE) equilibrated and run with buffer C. The protein purity was checked by SDS-PAGE and then concentrated to $7-10 \mathrm{mg} / \mathrm{ml}$ with a Vivaspin concentrator. The protein concentration was determined with the Bradford assay (see appendix A.3. 
Table 2.4.: Buffers for protein purification

Lysis buffer

$20 \mathrm{mM}$ Hepes pH 7.3

$50 \mathrm{mM} \mathrm{NaCl}$

$0.2 \mathrm{mM}$ PMSF

$1 \mathrm{mM}$ DTT

EDTA-free protease-inhibitor-cocktail (RocHE)

\section{Buffer A}

$50 \mathrm{mM}$ Hepes pH 7.3

$30 \mathrm{mM} \mathrm{KAc}$

\section{Buffer B}

50 mM Hepes pH 7.3

$300 \mathrm{mM} \mathrm{KAc}$

\section{Buffer C}

20 mM Hepes pH 7.3

$100 \mathrm{mM} \mathrm{NaCl}$

$1 \mathrm{mM} \mathrm{MgAc}$

$0.5 \mathrm{mM}$ EDTA

$0.1 \mathrm{mM}$ EGTA

$1 \mathrm{mM}$ DTT

\subsection{TEV-cleavage to remove His-tag}

To remove the N-terminal His-tag from the protein the TEV cleavage-site was used. After size exclusion chromatography, the peak fraction was pooled and transferred into a $15 \mathrm{ml}$ Falcon tube. Then, the protein concentration was measured with a Bradford assay. $2 \mu \mathrm{g}$ TEV protease per $100 \mu \mathrm{g}$ protein were added and incubated on a shaker at room temperature overnight. A second size exclusion chromatography was performed to remove the TEV protease from the protein. 


\subsection{Crystallization approach}

Before the start of crystallization trials, the $\mathrm{PC}^{\mathrm{TM}}$ Pre-Crystallization Test (HAMPTON Research, Aliso Viejo, CA-US) was used to identify the optimal protein amount for crystallization. The protocol as described by the company was followed (Watson and O'Callaghan (2005)).

Crystallization trials were performed with various sparse-matrix screens using the sitting drop vapour diffusion method. Drops were set up in 96-well plates (Greiner Bio ONE GMBH, Frickenhausen, D) by automatically mixing (mosquito Crystal, TTP LABTECH Ltd., Hertfordshire, UK) $200 \mathrm{nl}$ each of the protein and the reservoir solution, which were equilibrated against $100 \mu \mathrm{l}$ reservoir solution. Plates were kept at $20{ }^{\circ} \mathrm{C}$, unless otherwise stated. Plates were frequently inspected for crystal growth with a light microscope (OLYMPUs SZX12, Hamburg, D) and a polarizing filter.

When initial crystals were found a grid screen was conducted to find the optimal crystallization condition. This means that always only one of the crystallization ingredients of the original condition was altered. For example, the $\mathrm{pH}$ of the buffer was changed in both directions (more acidic or more alkaline) or a different buffer was used altogether. The amount of salts or precipitant was varied as well while the other crystallization components remained unchanged. This method was conducted in 96-well as well as 24-well plates (Greiner Bio One GmBH).

\subsubsection{Crystal seeding}

To enhance crystal growth seeding solutions made up of small crystals or quasicrystals were prepared and used during crystal drop set up. These small crystal fragments in the solution act as nuclei to which protein molecules can attach to form bigger crystals. Crystals were broken up with a small glass probe (courtesy of Douglas InstruMENTS), and transferred into a $1.5 \mathrm{ml}$ E-cup containing $50 \mu \mathrm{l}$ of the reservoir solution and a seed bead (HAMPton RESEARCH). The crystal solution was vortexed for two minutes, stopping every $30 \mathrm{sec}$ to cool it on ice. Then, several dilutions were prepared and immediately frozen at $-20^{\circ} \mathrm{C}$. Usually a $1: 100$ or $1: 1000$ seeding solution was used for setting up drops in a 1:3:4 (seeding solution:reservoir solution:protein) ratio.

\subsubsection{Additive screening}

A small amount of an additive was added to an already established crystallization condition. It causes a slight change in the crystallization condition and hence might cause the formation of better diffracting crystals. 
Commercial additive screens containing salts, co-factors, amino acids etc. are available, but any spare matrix screen can be used as additive, as well. To do so, the favoured crystallization condition was transferred into the reservoir well of a 96-well plate. With the mosquito Crystal $200 \mathrm{nl}$ protein were mixed with $180 \mathrm{nl}$ of the reservoir solution. Then, $20 \mathrm{nl}$ each of one of the 96 crystal conditions of the Crystal Screen 1/2 (HAMPton ReseARCH) was mixed with the protein-reservoir solution and treated as mentioned above in section 2.8 .

\subsection{Crystal measurement}

Prior to data collection, crystals were harvested from the drops using mounted cryoloops (HAMPTON RESEARCH), briefly transferred to a cryoprotection buffer consisting of mother liquor supplemented with a cryoprotectant like PEG 400, and subsequently flash-cooled and stored in liquid nitrogen. The crystals were measured at beamline X10SA (Swiss Light Source, Paul Scherrer Institute, Villigen, CH) or at beamline P11 (Deutsches Elektronen-Synchrotron, PETRA III, Hamburg, D) under a constant stream of liquid nitrogen. Both beamlines feature a Pilatus detector (DECTRIS LTD., Baden, $\mathrm{CH}$ ) that enabled rapid data collection.

A multi-wavelength anomalous diffraction (MAD) measurement was conducted to determine the phases. Firstly, a fluorescence scan of a selenomethionine (SeMet) derivative crystal was performed to determine the absorption peak as well as the point of inflection and remote of the absorption curve of Se (Hendrickson et al. 1990). Then, three datasets were recorded at three different wavelengths.

\subsection{Structure determination}

\subsubsection{Processing the datasets}

All datasets recorded at the beamline were processed with the XDS program suite (Kabsch, 2010). In the case of MAD data it was necessary to unset the parameter FRIEDEL'S LAW because of anomalous scattering effects. At first, reflections were indexed and integrated, possible space groups were determined and statistics about the dataset were calculated. One output file of XDS, CORRECT.LP, gave statistics about the quality of the data and helped to determine a reasonable resolution cut-off. With $X S C A L E$ several datasets can be scaled relative to each other, which was used for the MAD datasets. To prepare the data for further use, they were converted in a suitable format using $X D S C O N V$. 


\subsubsection{Phase determination}

The phases were determined from a derivative dataset with the program suite SHELX (Sheldrick, 2010). At first, a name.hkl file was produced with XDSCONV. Then, XPREP (Bruker) was used to prepare the file for SHELXD/E by writing out several output files. The instruction file (name_fa.ins) and the anomalous data in name_fa.hkl were used by SHELXD to locate the heavy atoms (Se) and to calculate the phases of the heavy atom substructure. It also provided an output file containing the marker atom coordinates (name_fa.res). SHELXE calculated the phases of the protein using this file, the anomalous and native data (name.hkl, calculated in XPREP). The resulting electron density map name.phs was visualized in Coot.

\subsubsection{Computing a starting model with $A C M I$}

For the automated construction of a structure model the program suite ACMI (Automatic Crystallographic Map Interpretation) was used because it is specially designed for low-resolution maps (DiMaio et al., 2007). The program is command line based and consists of three consecutive steps to calculate a model (see documentation $A C M I$ ). At first, the electron density map (in name.map-format), the protein sequence (name.seq), and the crystal parameters (name.cryst) were given to SHFEAR. It searched the electron density map for a set of templates (5-mer) for each amino-acid in the protein sequence and calculated the probability distribution for each amino-acid at every location. The resulting files were used by the next program, $B P$. It refined the probabilities calculated by SHFEAR by computing the probability distribution of each amino-acid at every location in the map by taking the location of the other amino-acids into account as well. It calculated maps and a backbone model consisting of $\mathrm{C}_{\alpha}$-atoms only. In the final step, SIDECHAIN_PF used the information computed before to calculate a set of models that best explained the electron density map. Using the argument $-b$, 20 models were generated.

\subsection{Chemical cross-linking with $\mathrm{BS}^{3}$}

Chemical cross-linking provides a permanent link between proteins and can give information about the location of binding using mass spectrometry analysis following in-gel digestion (Rappsilber et al., 2000).

$70 \mathrm{pmol}$ of the purified protein complex $\mathrm{DCTN1}_{412-533}-\mathrm{IC}_{2-69}$ was incubated with $125 \mu \mathrm{M}$ and $250 \mu \mathrm{M}$ of the lysine specific crosslinker BS ${ }^{3}$ (Thermo Scientific) for 30 min at room temperature. Each cross-link experiment was performed four times to yield enough 
cross-linked protein for mass spectrometry. The reaction was stopped by adding SDS sample buffer; the protein bands were separated on a $17.5 \%$ SDS-PAGE and stained with Coomassie blue. The appropriate bands were cut from the gel with a clean scalpel, cut into small pieces, and placed into a $1.5 \mathrm{ml}$ Eppendorf tube. All four excised protein bands prepared with the same $\mathrm{BS}^{3}$ concentration were pooled into one Eppendorf tube. The gel pieces were washed twice with each $150 \mu \mathrm{l} 50 \mathrm{mM}$ ammonium bicarbonate $(\mathrm{ABC})$ and $150 \mu \mathrm{l} 50 \mathrm{mM}$ acetonitrile $(\mathrm{ACN})$ to remove Coomassie. For the digestion, the gel pieces containing the cross-linked proteins were covered with trypsin $(13 \mathrm{ng} / \mu \mathrm{l}$ in $10 \mathrm{mM} \mathrm{ABC}$ and $10 \% \mathrm{ACN})$ and incubated overnight at $37^{\circ} \mathrm{C}$.

Next day, the supernatant was removed from the gel pieces and collected in a new $1.5 \mathrm{ml}$ Eppendorf tube. Then, the proteins were extracted from the gel pieces in three steps. First, the gel slices were incubated with water $(10 \mu \mathrm{l} /$ slice $)$ followed by ACN (80 $\mu \mathrm{l} /$ slice). Then, the gel pieces were incubated with $5 \%$ acetic acid $(65 \mu \mathrm{l} / \mathrm{slice})$ followed by ACN (50 $\mu \mathrm{l} /$ slice). This was followed by a final step of ACN (40 $\mu \mathrm{l} /$ slice). The respective supernatants were collected after every step. Each added solution was incubated for $15 \mathrm{~min}$ at $37^{\circ} \mathrm{C}$, at $1050 \mathrm{rpm}$ in the dark. The total supernatant was concentrated to $6 \mu \mathrm{l}$ in speed vac. An equal amount of a $10 \% \mathrm{ACN}$ and $0.2 \%$ formic acid solution was added to the sample, which was then subjected to mass spectrometry (LTQ Orbitrap Velos, Thermo ScIEnTIFIC).

The results were analysed with the $p$ Link software (Yang et al., 2012). 



\section{Results}

\subsection{Cloning and protein purification of various dynein-dynactin constructs}

Several DCTN1-IC constructs were cloned based on studies by Siglin et al. (2013) and Morgan et al. (2011) to address the question of dynein-dynactin interaction. Figure 3.1 contains an overview of the various constructs. The DCTN1-fragment length was kept in all plasmids while the length of IC changed. In all plasmids, except pMK243, DCTN1 $1_{412-533}$ contained an N-terminal His-tag for the purification with an Ni-NTA column.

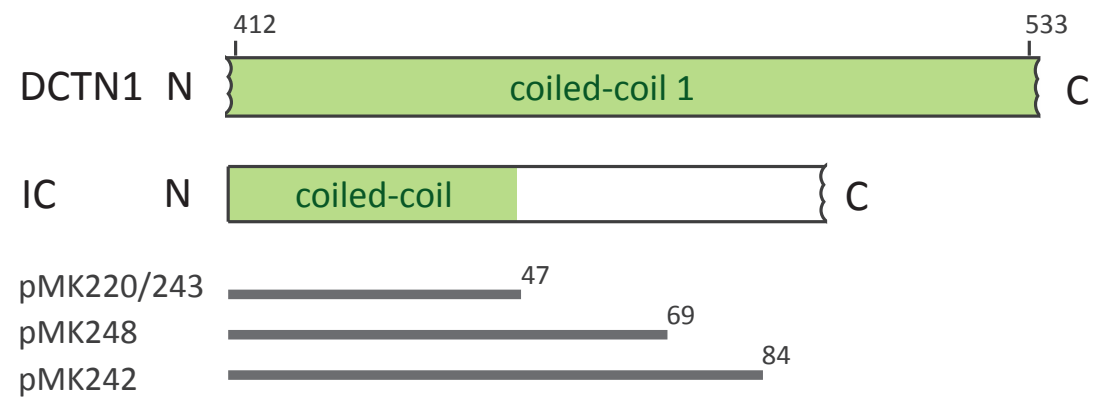

Figure 3.1.: Overview of all DCTN1-IC constructs. All constructs contain DCTN1 $1_{412-533}$. The length of the N-terminal IC fragment, however, changes in the various constructs. In contrast to the other plasmids, pMK243 has the N-terminal His-tag attached to IC and not DCTN1.

\subsection{1. $\mathrm{DCTN} 1_{412-533}-\mathrm{IC}_{2-47}$}

For the first construct (pMK220) the DCTN1 and IC fragment sizes were based on the study by Siglin (2010). Both fragments were cloned into the expression vector pRSFmD1mako (see figure B.1), which allowed a simultaneous expression of the proteins. The plasmid contains two multiple cloning sites (MCS) each having an Nterminal $6 \times$ His-tag for the purification with a Ni-NTA column. Human $\mathrm{IC}_{2-47}$ was amplified with primers PF7 and PF8b using plasmid pMK186 as template. Likewise, chicken DCTN1 $1_{412-533}$ was amplified with plasmid pMK146b as template and primers 
PF9 and PF10b. $\mathrm{IC}_{2-47}$ and $\mathrm{DCTN1} 1_{412-533}$ were ligated into the multiple cloning sites through the restriction sites NcoI/ HindIII (MCS1) and MluI/ BsrGI (MCS2), respectively. The use of $N c o \mathrm{I}$ for $\mathrm{IC}_{2-47}$ resulted in the removal of the His-tag for this protein fragment whereas the choice of restriction enzymes for DCTN1 ${ }_{412-533}$ generated an N-terminal His-tag. The cloning steps were carried out in E. coli XL-2 blue. After a positive clone was determined, the plasmid was transformed into the expression strain E. coli BL21-CodonPlus(DE3)RIL. An expression test in LB-medium supplemented with kanamycin $(25 \mu \mathrm{g} / \mu \mathrm{l})$ and chloramphenicol $(34 \mu \mathrm{g} / \mu \mathrm{l})$ and induced with $0.1 \mathrm{mM}$ IPTG (end-concentration) demonstrated that the protein complex was already soluble at $37^{\circ} \mathrm{C}$ (see figure 3.2 ). However, usually the protein was expressed at $30{ }^{\circ} \mathrm{C}$ to avoid an overgrowth of the $E$. coli culture.

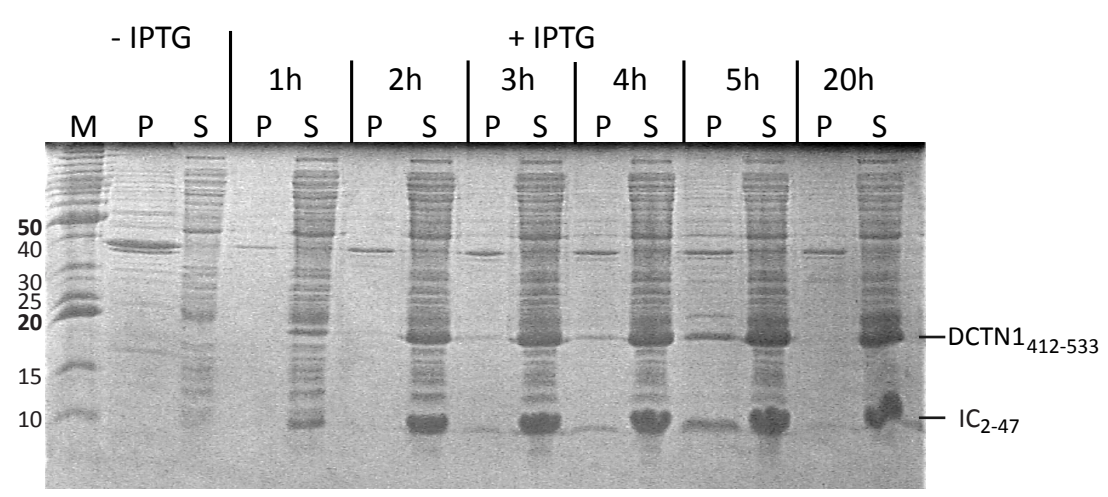

Figure 3.2.: $\mathrm{DCTN1} 1_{412-533}-\mathrm{IC}_{2-47}$ expression test at $37^{\circ} \mathrm{C} . \mathrm{M}=$ Protein marker, $\mathrm{P}=$ Pellet, $\mathrm{S}=$ Supernatant; Samples were taken before IPTG induction and after $1,2,3,4,5$, and 20 hours. Pellet and supernatant of each sample were applied on a $17.5 \%$ SDS PAGE gel.

The purification of the protein complex was carried out with a Ni-NTA column followed by size-exclusion chromatography as described in section 2.6 (see figure 3.3). Further experiments revealed, that adding $5 \%$ glycerol to buffer $\mathrm{C}$ for the dialysis and gelfiltration produced a more stable protein complex. This was apparent in SDS gels where the lower IC protein band was more pronounced. However, IC always produced a fainter protein band than DCTN1.

In an attempt to enrich $\mathrm{IC}_{2-47}$ the His-tag between IC and DCTN1 was swapped (pMK243). Firstly, a PCR with primers PF9-ohneHis and PF10b and plasmid pMK146b as template was conducted to amplify $\mathrm{DCTN1} 1_{412-533}$. The PCR product was ligated into vector pRSFmD1mako over the restriction sites $M l u \mathrm{I}$ and $N d e \mathrm{I}$, which resulted in a His-tag free $\mathrm{DCTN1}_{412-533}$. Then, His- $\mathrm{IC}_{2-47}$ was amplified with PF7-HisTev and PF8b and pMK186 as template. The resulting PCR product contained a $8 \times$ His-tag 


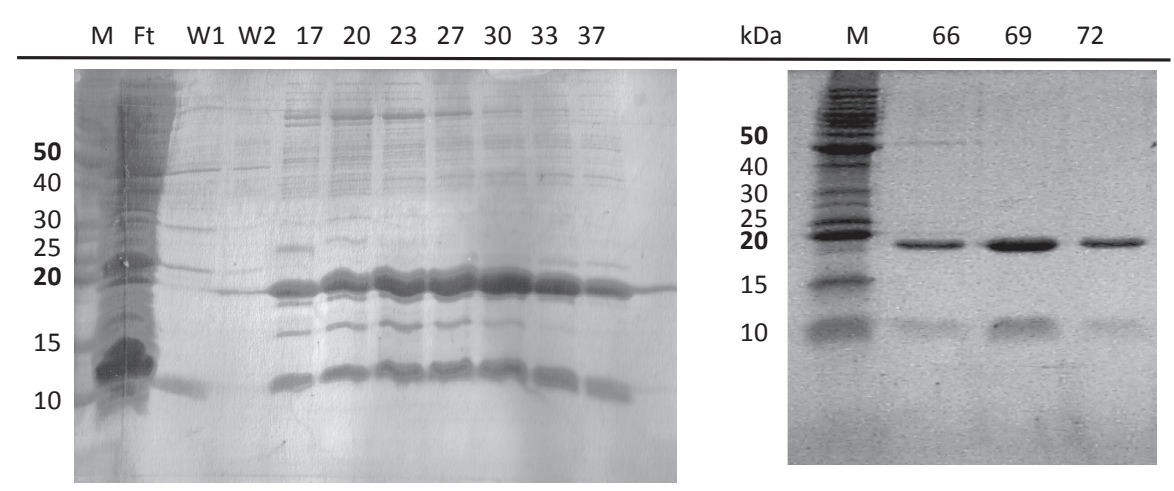

Figure 3.3.: SDS-PAGE gels of DCTN1 ${ }_{412-533}-\mathrm{IC}_{2-47}$ purification. (Left) $17.5 \%$ SDS-PAGE gel after Ni-NTA purification. $\mathrm{Ft}=$ flowthrough, $\mathrm{W} 1=$ first washing step with buffer A1 and A2, W2 = second washing step with buffer A1 supplemented with $40 \mathrm{mM}$ imidazole (Right) $17.5 \%$ gel after gelfiltration. Above the gels the fraction numbers are given and on the left the size of the respective protein marker lanes.

and was also cloned into the pRSFmD1mako vector already containing DCTN1 ${ }_{412-533}$. Verification of the cloned PCR products was carried out with restriction digestion. A positive plasmid was transformed into E. coli BL21-CodonPlus(DE3)RIL and then protein purification was performed (see figure 3.4) as described for plasmid pMK220. In contrast to $\mathrm{DCTN1} 1_{412-533}-\mathrm{IC}_{2-47}$, gelfiltration resulted in three distinct peaks; fractions 66-76, 86-97 and 103-113. The first two peak fractions were pooled separately and dialysed against buffer $\mathrm{C}$. Half the volume of the first peak was then concentrated to $6.8 \mathrm{mg} / \mathrm{ml}$ for crystallization trials. The other half was mixed together with the second peak fractions and the His-tag was cleaved from $\mathrm{IC}_{2-47}$ as described in section 2.7 because of the potential interference of the tag with the protein interaction. This was followed by a second gelfiltration to remove TEV protease from the sample. Again, three peaks were visible in the chromatogram, which were made up of fractions 64-74, 99-109, and 115-120. Only fractions 66-74 were pooled and concentrated to $7 \mathrm{mg} / \mathrm{ml}$ with a Vivaspin MWCO 5000 since it was the only peak containing both proteins. 

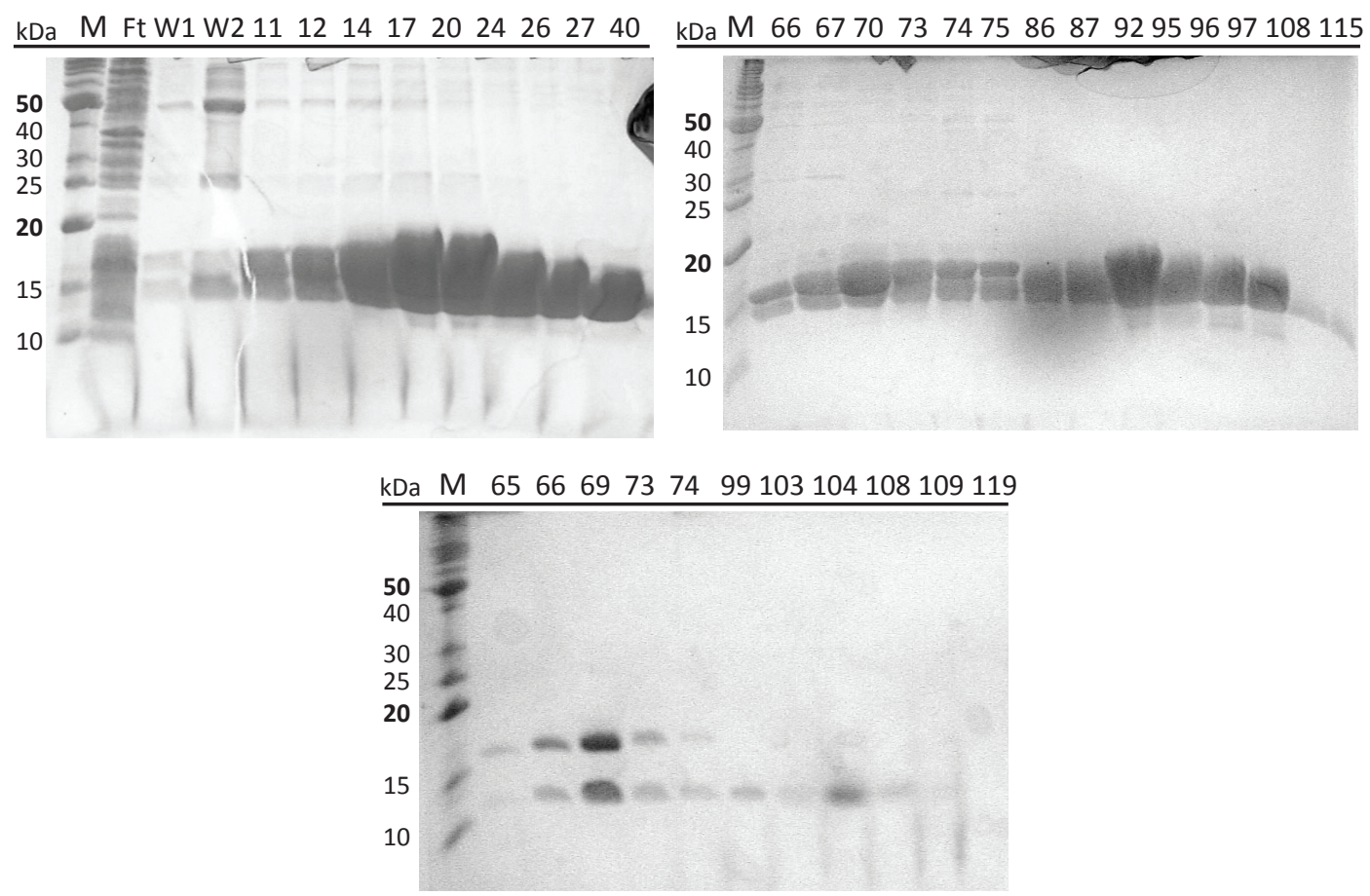

Figure 3.4.: SDS-PAGE gels of DCTN1 ${ }_{412-533} /$ His-TEV-IC ${ }_{2-47}$ purification. (Left) $17.5 \%$ SDS gel after Ni-NTA purification. Ft = flowthrough, W1 = first washing step with buffer A1 and A2, W2 = second washing step with buffer A1 supplemented with $40 \mathrm{mM}$ imidazole (Right) $17.5 \%$ gel after first gelfiltration. (Bottom) 17.5\% SDS gel from second gelfiltration after TEVcleavage. Above the gels the fraction numbers are given and on the left the size of the respective protein marker lanes.

\subsection{2. $\mathrm{DCTN} 1_{412-533}-\mathrm{IC}_{2-84}$}

Morgan et al. (2011) demonstrated with an NMR titration experiment using recombinantly expressed Drosophila melanogaster proteins that two regions of IC bind to DCTN1. Transferring this finding onto human IC (see figure 3.5) resulted in a longer IC fragment $\left(\mathrm{IC}_{2-84}\right)$ in the new construct (pMK242).

Initially, the longer IC fragment was amplified with primers PF7 and PF8-2 using pMK186 as template. The shorter IC fragment in pMK220 was cut out using the former ligation sites $\mathrm{Nco} / \mathrm{I}$ indIII. The new $\mathrm{IC}_{2-84}$ was then cloned into the vector over the same restriction sites. The solubility of the protein complex was verified in E. coli BL21-CodonPlus(DE3)RIL with an expression test at $37{ }^{\circ} \mathrm{C}$ as described in 3.1.1. This was followed by the first purification step over a Ni-NTA column. Fraction inspection on SDS-PAGE gel showed highly degraded protein bands for both DCTN1 and IC (data not shown). The purification protocol was modified to improve protein 
region 1

region 2

Dm 1 ----MDRKAELERKKARLAALREERDRRRREREIRDMEEAA--GRIGGGAGIDRDCRKDIDEMLSSIGVAPVSEVISSISSVNSMTSDNSNT 86 HS 1 MSDKSDLRAELERKKCRLACIREEKRRKEEERKKKEADMCCKKEPVCDDSLDR-KRRETEALICSIGISPEP P-VPTPMSPSSKSVSTPS 90 IC $2-47$ I

IC 2-69

IC 2-84

Figure 3.5.: Alignment of dynein intermediate chains. $\mathrm{Dm}=$ Drosophila melanogaster, $\mathrm{Hs}=$ Homo sapiens; The alignment of the N-terminal part of $D$. melanogaster and human dynein intermediate chain is shown. The grey bars represent the two binding regions found in D. melanogaster by Morgan et al. (2011). The blue bars depict the length of the various IC constructs designed in this work.

stability mainly by changing the $\mathrm{pH}$ of the buffers from 7.3 to 8.0. For the lysis of cells the buffer was changed to $25 \mathrm{mM}$ Hepes (pH 8.0), $200 \mathrm{mM} \mathrm{NaCl}$, and $20 \mathrm{mM}$ imidazole ( $\mathrm{pH}$ 8.0) (for reference of standard buffer see section 2.4). After the sample was applied onto the column, it was washed with 20 column volumes buffer A1 (25 mM Hepes (pH 8.0), $200 \mathrm{mM} \mathrm{NaCl}$, and $20 \mathrm{mM}$ imidazole ( $\mathrm{pH} 8.0$ )), followed by 20 column volumes buffer A1 supplemented with $40 \mathrm{mM}$ imidazole ( $\mathrm{pH} 8.0$ ), and finally eluted using a linear gradient of 60 to $500 \mathrm{mM}$ imidazole over 6-8 column volumes. The peak fractions were visualised on SDS gel (17.5\%) (see figure 3.6), pooled and dialysed in buffer $\mathrm{C}$ to remove imidazole. Again, the buffer (see section 2.4 for composition) was altered to stabilize the protein by adding $25 \mathrm{mM}$ Hepes ( $\mathrm{pH} 8.0$ ) and $150 \mathrm{mM} \mathrm{NaCl}$ instead of $20 \mathrm{mM}$ Hepes ( $\mathrm{pH} \mathrm{7.3)}$ and $100 \mathrm{mM} \mathrm{NaCl}$, and by supplementing with $5 \%$ glycerol. Despite the change of buffer the $\mathrm{DCTN1} 1_{412-533}-\mathrm{IC}_{2-84}$ complex still proved to be quite unstable and precipitated within 16 hours after being concentrated with a Vivaspin MWCO 5000 to $8-10 \mathrm{mg} / \mathrm{ml}$. This meant that crystallization trials had to be set up right away. Furthermore, the expression level of this construct was considerably lower in comparison to the other constructs. 

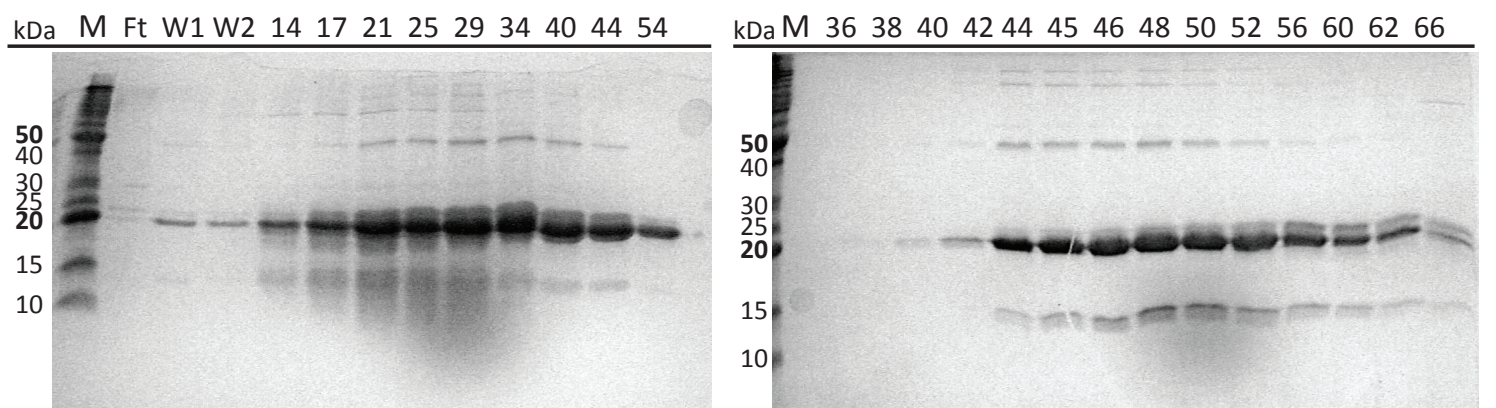

Figure 3.6.: SDS-PAGE gels of DCTN1 ${ }_{412-533}-\mathrm{IC}_{2-84}$ purification. (Left) $17.5 \%$ SDS-PAGE gel after Ni-NTA purification. Ft = flowthrough, W1 = first washing step with buffer A1, W2 = second washing step with buffer A1 supplemented with $40 \mathrm{mM}$ imidazole; Fractions 14 to 54 were pooled for further use. (Right) $17.5 \%$ gel after gelfiltration. Above the gels the fraction numbers are given and on the left the size of the respective protein marker lanes.

\subsection{3. $\mathrm{DCTN} 1_{412-533}-\mathrm{IC}_{2-69}$}

The fast precipitation of $\mathrm{DCTN} 1_{412-533}-\mathrm{IC}_{2-84}$ led to the consideration that the extension from 47 aa to 84 aa might have been too long. A construct was designed (pMK248) that terminated before the stretch with several prolines (for more details see discussion 4.1). $\mathrm{IC}_{2-69}$ was amplified with primers PF7 and PF8-3 and pMK186 as template. The plasmid pMK220 was digested with $N c o \mathrm{I} / H i n d I I I$ to remove $\mathrm{IC}_{2-47}$ and to ligate the newly amplified IC fragment into the plasmid. After transforming a positive plasmid into $E$. coli BL21-CodonPlus(DE3)RIL a protein expression test at $37^{\circ} \mathrm{C}$ demonstrated that the protein complex was soluble. Protein purification was carried out in the exact same manner as for $\mathrm{DCTN1} 1_{412-533}-\mathrm{IC}_{2-47}$ and the purified complex showed a similar stability (see figure 3.7). The protein complex was concentrated with a Vivapsin MWCO 5000 to $7-10 \mathrm{mg} / \mathrm{ml}$. 

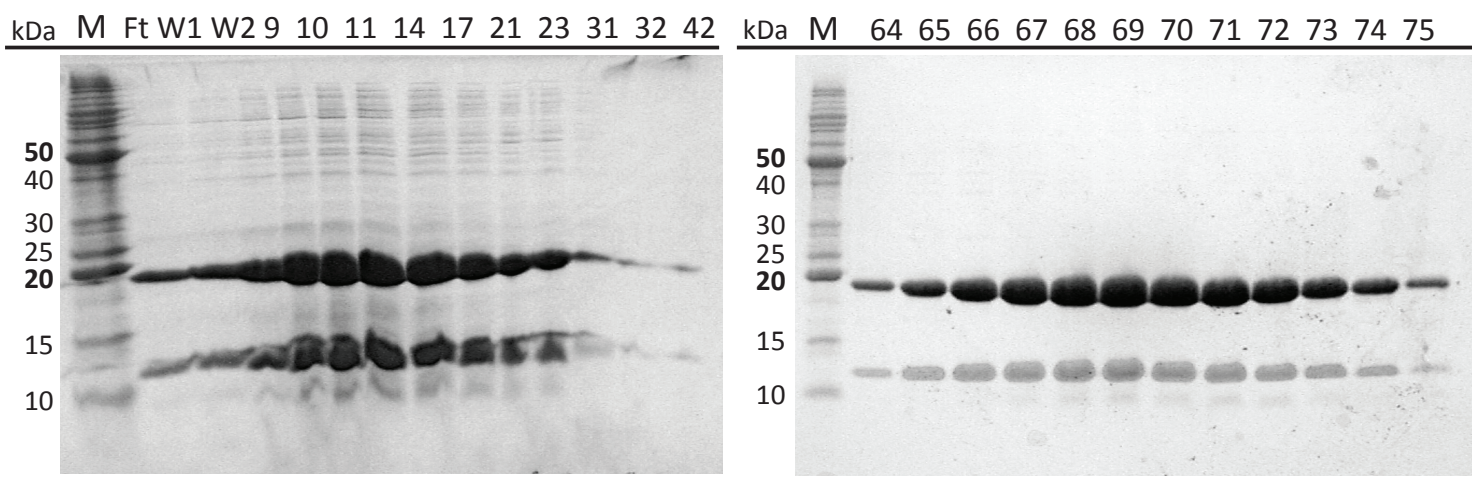

Figure 3.7.: SDS-PAGE gels of DCTN1 ${ }_{412-533}-\mathrm{IC}_{2-69}$ purification. (Left) $17.5 \%$ SDS-PAGE gel after Ni-NTA purification. $\mathrm{Ft}=$ flowthrough, $\mathrm{W} 1=$ first washing step with buffer A1 and A2, W2 = second washing step with buffer A1 supplemented with $40 \mathrm{mM}$ imidazole (Right) 17.5\% SDS-PAGE gel after gelfiltration. Above the gels the fraction numbers are given and on the left the size of the respective protein marker lanes in $\mathrm{kDa}$.

\subsection{Crystallization of the various DCTN1-IC constructs and data collection}

In general the initial search for crystallization conditions was performed with the sitting drop method in 96-well plates using various sparse matrix screens (Wizard I-IV, Crystal Screen 1+2, Protein Complex Suite, Morpheus, JCSG Core II). $100 \mu$ l of a crystallization condition was pipetted into the reservoir well using a pipetting robot (Tecan). Protein and reservoir solution were mixed in a ratio of 1:1 (200 nl each). Plates were kept at $20{ }^{\circ} \mathrm{C}$ and frequently checked for crystals.

\subsubsection{Crystallization of DCTN1 $1_{412-533}-\mathrm{IC}_{2-47}$ construct}

Initial screening was performed by Christian Eckert. $4 \mathrm{mg} / \mathrm{ml}$ were used for screening in 96-well plates. Tiny spherulites were found in condition 16 (0.2 M sodium chloride, 0.1 M MES pH 6.5, 10\% PEG 4000) of the Protein Complex Suite (Quiagen). This was followed by different grid screens in 24-well plates using the hanging drop method in which various buffers, salts and PEG concentrations were tested. There, two crystallization conditions were found that yielded two different crystal forms after 3-5 days, house-like (5\% PEG 8000, Hepes pH 7.5) and trapezium-like crystals (11\% PEG 1000, $150 \mathrm{mM}$ ammonium sulphate, $0.1 \mathrm{M}$ Tris $\mathrm{pH} 8.5$ ), which can be seen in figure 3.8 A. Both crystal forms were frozen in different cryoprotectants and measured at beamline PXII at the Swiss lightsource in Villigen $(\mathrm{CH})$. The best crystal belonging 
to the house-like form diffracted up to $6 \AA$. The trapezium-like crystals displayed even lower diffraction qualities. Following this finding, further grid screens were performed using $10 \mathrm{mg} / \mathrm{ml}$ protein. This resulted in a crystallization condition (6\% PEG 4000, $300 \mathrm{mM}$ ammonium sulphate, $0.1 \mathrm{M}$ Na-citrate $\mathrm{pH} 5.5,5 \%$ glycerol) in which bigger house-like crystals with edge lengths of 100-200 $\mu \mathrm{m}$ (see figure 3.8 B) appeared after 3-5 days. Initially, the best crystal contained 15\% PEG 400 as cryoprotectant and diffracted to a resolution of $3.5 \AA$.

Much later native crystals grew in $0.1 \mathrm{M}$ Na-citrate pH 5.5, 5\% PEG 4000, and $0.1 \mathrm{M}$ ammonium sulphate. As cryoprotectant $15 \%$ glycerol was added to the crystal condition and measurements were conducted at beamline P11 at PETRA III, Hamburg. The best crystal diffracted to $2.9 \AA$.

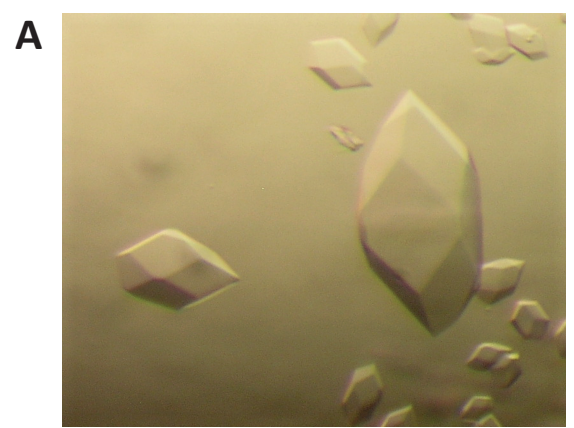

11\% PEG 1000, $150 \mathrm{mM}$ ammonium sulfate, $0.1 \mathrm{M}$ Tris $\mathrm{pH} 8.5$

B

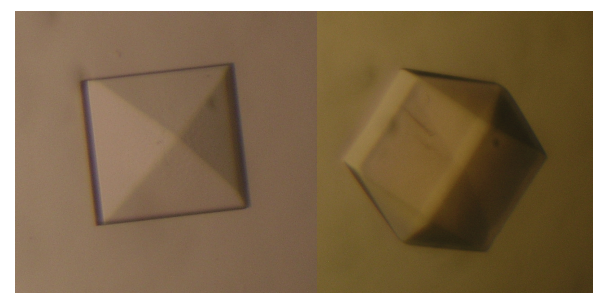

$6 \%$ PEG 4000, $300 \mathrm{mM}$ ammonium sulfate, $0.1 \mathrm{M}$ Na-citrat pH 5.5, 5\% glycerol

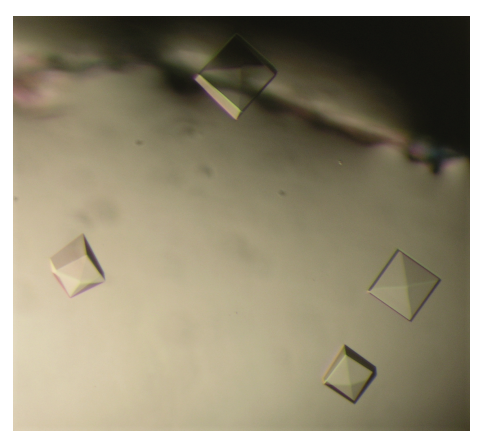

5\% PEG 8000, $0.1 \mathrm{M}$ Hepes $\mathrm{pH} 7.5$

C

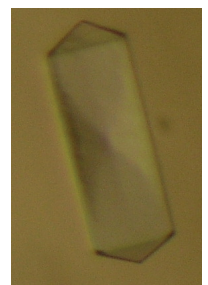

5\% PEG 4000, $300 \mathrm{mM}$ ammonium sulfate, $0.1 \mathrm{M}$ Na-citrat pH 5.5, 5\% glycerol + 10\% Additive

Figure 3.8.: Crystals of DCTN1 ${ }_{412-533}-\mathrm{IC}_{2-47}$ (A) These are the first crystals which formed in the initial grid screen. The crystals in the left image display a trapezium form whereas crystals on the right were denoted as house-like. (B) This image shows the house-like crystal form in the final crystal condition. (C) The crystals found in the additive screening showed an elongated version of the house-like form. However, this did not affect resolution.

In search of better diffracting crystals several strategies were followed. For example, an additive screening was performed as described in subsection 2.8.2, $10 \%$ of Crystal Screen 1+2 (Hampton Research) and Wizard 1+2 (Emerald BioStructures) were added to the protein-reservoir (6\% PEG 4000, $300 \mathrm{mM}$ ammonium sulphate, $0.1 \mathrm{M}$ Na-citrate pH 5.5, 5\% glycerol) drops. After one day the first small crystals appeared 
in a drop with condition 48 from Crystal Screen 2 (10\% PEG 20,000, $0.1 \mathrm{M}$ bicine (pH 9) and $2 \%$ dioxane) as additive. They looked like an elongated version of the house-like form. In 24-well plates grid screens were set up to scan for better crystallization conditions. The reservoir composition as well as the amount of condition 48 were varied and resulted in bigger elongated crystals as shown in figure $3.8 \mathrm{C}$. Measurements at beamline PXII at the SLS (Villigen, $\mathrm{CH}$ ) showed that none of the elongated crystals diffracted better than $3.5 \AA$.

Another strategy was to change the buffer (Na-citrate) while the rest of the original crystallization condition was kept. Buffers imidazole ( $\mathrm{pH} 5.4$ ), Bis-Tris ( $\mathrm{pH} 5.6$ ) and $\mathrm{Na}$-acetate ( $\mathrm{pH} 5.4$ ) were chosen for this purpose. No crystals appeared in drops set up with Bis-Tris but the other two buffers produced crystals similar to the house-like form. However, x-ray measurement at the SLS showed no improvement in resolution.

Furthermore, crystallization trials were set up with protein concentrated after dialysis and leaving out gelfiltration to have more $\mathrm{IC}_{2-47}$ bound to DCTN1 $1_{412-533}$. A first set up using the sparse matrix screen Protein Complex Suite and seeding with DCTN1 $1_{412-533^{-}}$ $\mathrm{IC}_{2-47}$-seed revealed crystals in several conditions with pHs between 7 and 8 . Grid screens with $0.1 \mathrm{M}$ Hepes $\mathrm{pH} 7.3$ or Tris $\mathrm{pH} 8$ were prepared in 24 -well plates. Crystals appeared in conditions containing $0.1 \mathrm{M}$ Hepes $\mathrm{pH}$ 7.3, $0.1 \mathrm{M}$ magnesium acetate and 2-7\% PEG 8000 after 3-5 days. They formed the same house-like crystals. Subsequently, crystals were frozen in their mother liquor containing $20 \%$ glycerol and then measured at beamline P11 at PETRA III, Hamburg. The best crystal diffracted up to $3.4 \AA$.

Crystallization with DCTN1 $1_{412-533}-\mathrm{IC}_{2-47}-\mathrm{His}-\mathrm{Tev}$ was split in two parts. On the one hand, protein from the first gelfiltration $(7 \mathrm{mg} / \mathrm{ml})$ and on the other hand, protein with the His-tag cleaved of $\mathrm{IC}_{2-47}(10 \mathrm{mg} / \mathrm{ml})$ were applied to various crystal screens. In both cases, crystallization was facilitated by adding crystal-seed (see subsection 2.8.1) derived from DCTN1 $1_{412-533}-\mathrm{IC}_{2-47}$ crystals to the protein-reservoir drop. The 96-well plates were checked frequently for crystals. After 4 months crystals were found in several crystallization conditions (see figure 3.9) in the set up with the His-tag-containing protein complex. The conditions in which crystals appeared differ in their buffers and their $\mathrm{pH}$ but all contain high amounts of long-chained PEGs (Protein Complex Suite $\rightarrow$ 29: 0.1 M Tris pH 8.0, 20\% PEG 4000; 36: 0.2 M Potassium iodide, 0.1 M MES pH 6.5, 25\% PEG 4000; 61: 0.1 M Hepes pH 7.0, 20\% PEG 8000). Because the crystals were grown together on the base they were separated before being frozen in mother 
liquor containing $15 \%$ glycerol. This was followed by measurements at beamline P11 at PETRA III in Hamburg. Diffraction was no better than $20 \AA$.
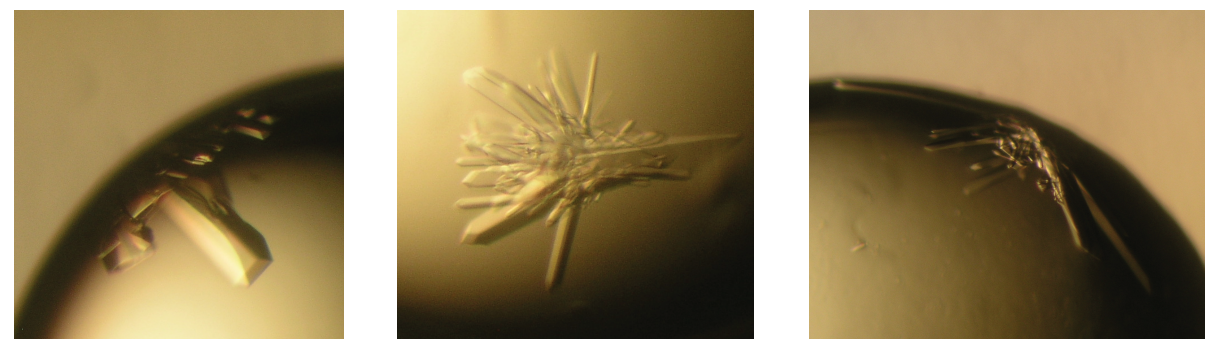

Figure 3.9.: Crystals from DCTN1 ${ }_{412-533}-\mathrm{IC}_{2-47}-\mathrm{His}-\mathrm{Tev}$ These crystals all grew in conditions from Protein Complex Suite. They all formed elongated crystals which were grown together on the base. (Left) These crystals grew in $0.1 \mathrm{M}$ Tris $\mathrm{pH} 8.0$ and 20\% PEG 4000. (Center) This crystal condition contains 0.2 M Potassium iodide, 0.1 M MES pH 6.5, and 25\% PEG 4000. (Right) The last crystal was found in $0.1 \mathrm{M}$ Hepes pH 7.0, and 20\% PEG 8000.

\subsubsection{Crystallization of DCTN1 $1_{412-533}-\mathrm{IC}_{2-47}$ with incorporated selenomethionine}

In order to solve the phase problem protein with incorporated selenomethionine instead of the usual methionine was produced. Each DCTN1 $1_{412-533}$ fragment contains 4 methionine positions and $\mathrm{IC}_{2-47}$ features one position in which selenomethionine could be incorporated. As a rule of thumb 1 selenomethionine per 100 amino acids is sufficient for conducting multi-wavelength anomalous diffraction measurements (Hendrickson and Ogata, 1997). Protein expression and purification were carried out as described in subsection 2.4.3. The protein complex was concentrated with a Vivaspin 5000 to $8 \mathrm{mg} / \mathrm{ml}$ and crystallization trials were set up in 24-well plates using the native crystallization condition as a starting point for grid screens. Crystals formed in 5\% PEG 4000, $150 \mathrm{mM}$ ammonium sulphate, $0.1 \mathrm{M}$ Na-citrate $\mathrm{pH}$ 5.5, and 5\% glycerol and they looked like the crystals derived from native protein (see figure $3.8 \mathrm{~B}$ ). For x-ray measurements crystals were shortly soaked in a cryoprotectant (mother liquor $+15 \%$ PEG 400) and frozen in liquid nitrogen. The derivative crystals were measured at beamline PXII at the SLS in Villigen $(\mathrm{CH})$ and the best crystal diffracted to $3.4 \AA$. 


\subsubsection{Crystallization of DCTN1 $1_{412-533}-\mathrm{IC}_{2-84}$ construct}

Fresh protein $(8 \mathrm{mg} / \mathrm{ml})$ was used to set up crystallization trials in 96 -well plates using various sparse matrix screens (Wizard 1+2, Protein Complex Suite, Crystal Screen $1+2$, Morpheus, JCSG Core II). Plates were checked regularly but crystals or crystallike structures did not form.

\subsubsection{Crystallization of $\mathrm{DCTN} 1_{412-533}-\mathrm{IC}_{2-69}$ construct}

Crystallization was carried out with concentrated protein after dialysis $(9 \mathrm{mg} / \mathrm{ml})$ and after gelfiltration $(8 \mathrm{mg} / \mathrm{ml})$. Various sparse matrix screens were applied. Crystals were found only in a set up with protein used after dialysis (Wizard $3+4 \rightarrow 52: 0.2 \mathrm{M}$ ammonium sulphate, $0.1 \mathrm{M}$ BisTris $\mathrm{pH}$ 5.5). The shape of these crystals was the same as found in DCTN1 $1_{412-533}-\mathrm{IC}_{2-47}$ (see figure 3.8 C). After freezing the crystals in their mother liquor supplemented with 15\% PEG 400, the crystals were measured at beamline P11 at PETRA III (Hamburg). They showed reflections up to $6 \AA$ A. Furthermore, dissolving some of the crystals in water and running the sample on $17.5 \%$ SDS gel only showed a protein band for DCTN1 $1_{412-533}$ and not IC.

\subsection{Data processing}

Data processing was performed with $X D S($ Kabsch, 2010 $)$ to reduce data and to determine cell parameters and the space group (see subsection 2.10.1). Native and derivative protein crystallized in the primitive tetragonal space group $\mathrm{P} 4_{1} 2_{1} 2$. The determination of the exact space group resulted from the examination of systematic absences of reflections. The absence of reflections for 001: $=4 n+1,4 n+2$, and $4 n+3$ and for $h 00: h=$ $2 \mathrm{n}+1$ led to the conclusion that the crystals either belonged to space group $\mathrm{P}_{3} 2_{1} 2$ or $\mathrm{P} 4_{1} 2_{1} 2$. Since those two space groups are enantiomer to each other only the successful solution of the phase did help to determine $\mathrm{P} 4_{1} 2_{1} 2$ as the true space group.

The best measured derivative $\mathrm{DCTN}_{412-533}-\mathrm{IC}_{2-47}$ dataset diffracted until $3.4 \AA$ and had the following cell parameters: $\mathrm{a}=\mathrm{b}=156.34 \AA, \mathrm{c}=131.14 \AA, \alpha=\beta=\gamma=$ $90^{\circ}$. The dataset consisted of 360 diffraction images taken at an oscillation range of $0.5^{\circ}$. 262,952 reflections were observed of which 42,432 reflections were unique. This equates to a multiplicity of 6.2. In the highest resolution shell (3.6-3.4 $\AA$ ) it exhibited a signal-to-noise ratio of 1.87 . The completeness of data amounts to $99.4 \%$ (see figure 3.10 . 


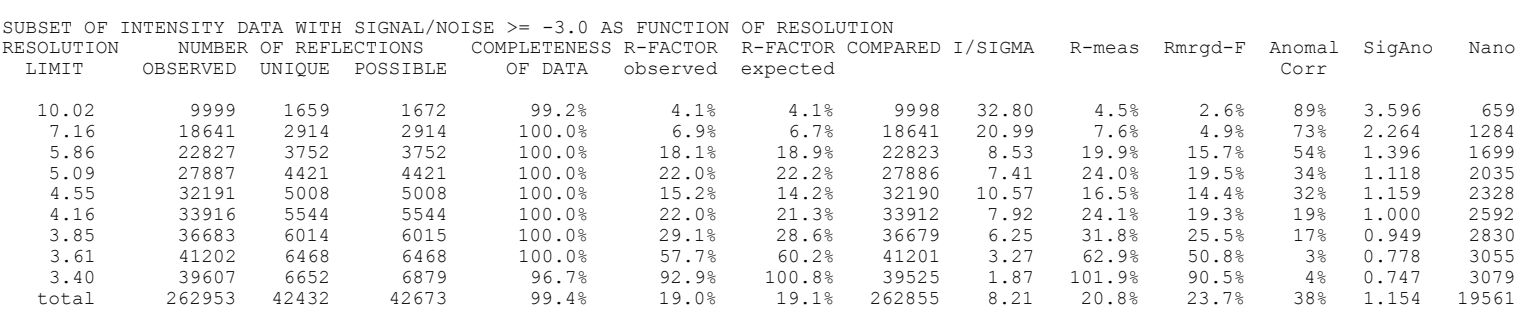

Figure 3.10.: Processing statistics of derivative DCTN1 $1_{412-533}-\mathrm{IC}_{2-47}$ Excerpt was taken from CORRECT.LP.

The best native crystal derived from the DCTN1 $1_{412-533}-\mathrm{IC}_{2-47}$ construct and formed in 0.1 M Na-citrate pH 5.5, 5\% PEG 4000, $0.1 \mathrm{M}$ ammonium sulphate with a proteinto-reservoir solution ratio of 1:2. It had a resolution of $2.9 \AA$ and displayed slightly different cell parameters $\left(\mathrm{a}=\mathrm{b}=155.32 \AA, \mathrm{c}=129.26 \AA, \alpha=\beta=\gamma=90^{\circ}\right)$. The processed dataset consisted of 1800 images taken at an oscillation range of $0.5^{\circ}$. The 935,917 observed reflections, which contained 35,585 unique reflections, equated to a multiplicity of 26.3. A signal-to-noise ratio of 1.78 and a $\mathrm{CC}_{1 / 2}$ of $73.4 \%$ could be found in the highest resolution shell (2.95-2.9 $\AA$ ) (see figure 3.11).

\begin{tabular}{|c|c|c|c|c|c|c|c|c|c|c|c|c|c|}
\hline $\begin{array}{l}\text { RESOLUTION } \\
\text { LIMIT }\end{array}$ & $\begin{array}{r}\text { NUMBE } \\
\text { OBSERVED }\end{array}$ & $\begin{array}{l}\text { R OF REE } \\
\text { UNIQUE }\end{array}$ & $\begin{array}{l}\text { LECTIONS } \\
\text { POSSIBLE }\end{array}$ & $\begin{array}{c}\text { COMPLETENESS } \\
\text { OF DATA }\end{array}$ & $\begin{array}{l}\text { R-FACTOR } \\
\text { observed }\end{array}$ & $\begin{array}{l}\text { R-FACTOR } \\
\text { expected }\end{array}$ & COMPARED & I/SIGMA & $\mathrm{R}$-meas & $\mathrm{CC}(1 / 2)$ & $\begin{array}{l}\text { Anomal } \\
\text { Corr }\end{array}$ & SigAno & Nanc \\
\hline 40.00 & 130 & 10 & 11 & $90.9 \%$ & $2.8 \%$ & $3.3 \frac{0}{8}$ & 129 & 79.07 & $2.9 \%$ & $100.0 *$ & -12 & 0.828 & \\
\hline $3 c$ & 497 & 27 & 27 & 100 & & 3. & 497 & & 3. & & & 1 & 10 \\
\hline 20 & 1990 & 94 & 94 & & & & 1990 & & & & -4 & & \\
\hline 10.00 & 18593 & 848 & 848 & 100 & 2. & 3. & 185 & 94 & 2. & & - & 0.657 & 585 \\
\hline 6.00 & 79844 & 3284 & 328 & $99.9 \%$ & $3.8 \%$ & $4.1 \%$ & 79844 & 65.21 & $3.9 \%$ & $100.0 *$ & -6 & 0.795 & 2648 \\
\hline & 252804 & & & & & $7.5 \%$ & 252 & & & & - & & 83 \\
\hline 3.50 & 175635 & 6620 & 66 & 100. & $21.9 \%$ & $21.7 \frac{\circ}{\circ}$ & 175635 & 18.53 & $22.3 \%$ & 9 & -5 & 5 & 593 \\
\hline 3.00 & 315212 & 11739 & 1173 & 100. & $89.6 \%$ & 92.8 & 3152 & 4 & 91 & $95.9 *$ & 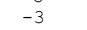 & 0 & 10675 \\
\hline 2.9 & 4435 & & 16 & 100. & 96. & 29 & & & 200. & 83 & -2 & & 1494 \\
\hline 2.90 & 59 & 3 & 1 & & $9.1 \%$ & & 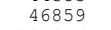 & 1. & $64.0 \%$ & 73.4 * & & & \\
\hline otal & 935917 & 35585 & 35602 & $100.0 \%$ & $9.9 \%$ & $10.3 \%$ & 916 & 25.37 & $10.1 \%$ & $100.0 *$ & -4 & & 3 \\
\hline
\end{tabular}

Figure 3.11.: Processing statistics of native DCTN1 $1_{412-533}-\mathrm{IC}_{2-47}$ Excerpt was taken from XSCALE.LP.

\subsection{Phase solution with selenomethionine}

A multi-wavelength anomalous diffraction measurement (MAD) of a crystal with incorporated selenomethionine was conducted to solve the phase problem. For this experiment a fluorescence scan was performed to determine the absorption peak and the point of inflection and remote of the absorption curve of Se. Then, a dataset each at wavelengths $0.979060 \AA$ (peak), $0.979650 \AA$ (inflection), and $0.971380 \AA$ (remote) of the same crystal was recorded. The datasets were processed with $X D S$ (Kabsch, 2010) as described in subsection 2.10.1,

The three datasets were merged and prepared for phase solution in XPREP. In order to determine a cut-off for the anomalous signal the anomalous signal-to-noise ratio was 
calculated for different resolution ranges for all three datasets. As a rule of thumb the data should be cut at the resolution where the anomalous signal-to-noise drops below 1.3. In figure 3.12 the processing statistics calculated by XPREP are shown and it demonstrates that in the remote dataset anomalous signal is only reliably detectable until $6 \AA$. The peak dataset, which was recorded first, exhibited anomalous signal up to $4.6 \AA$. At this point it got apparent that especially the last recorded remote dataset exhibited radiation damage. Therefore, instead of solving the phases with MAD, single-wavelength anomalous diffraction (SAD) was employed using the anomalous signal from the peak dataset alone.

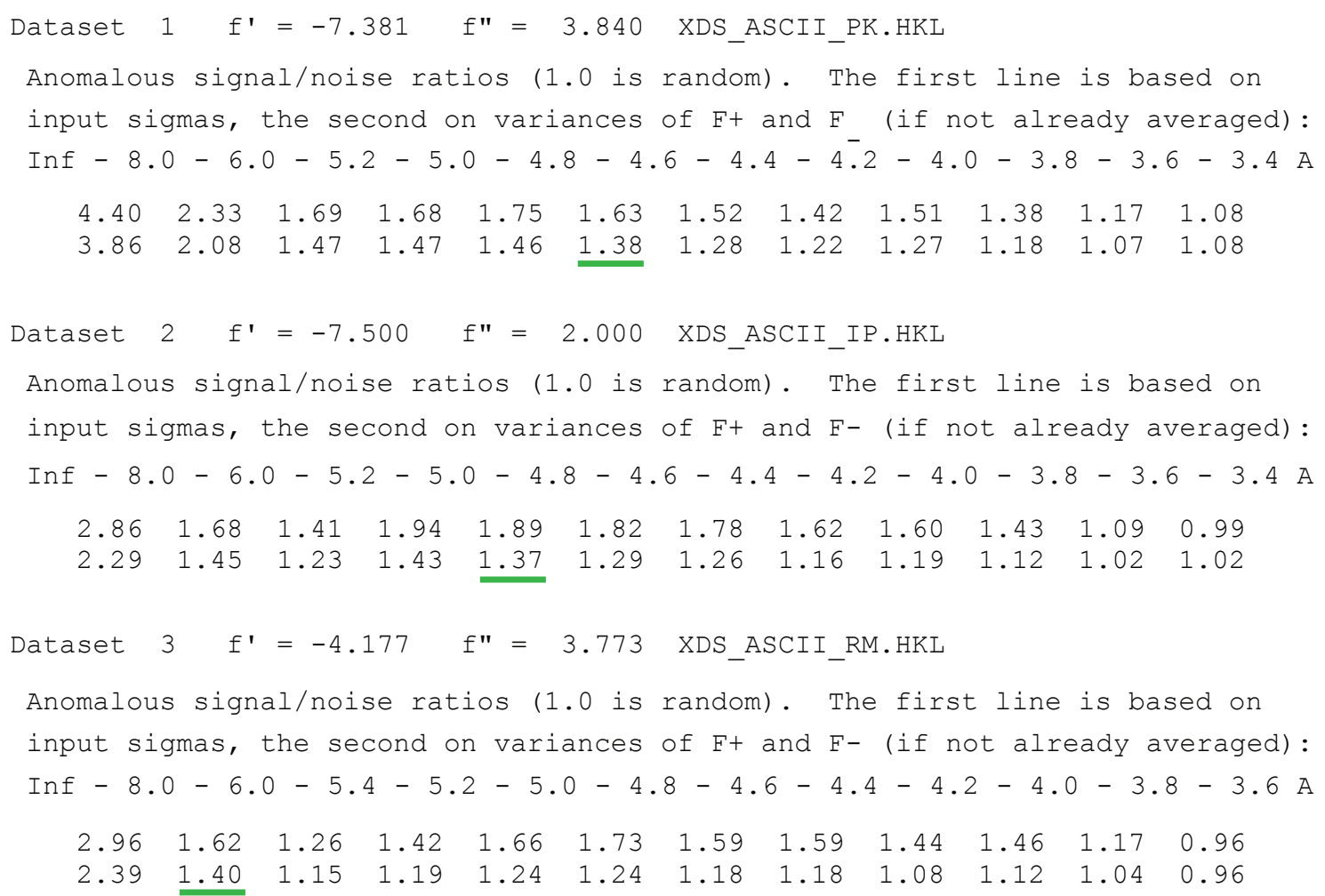

Figure 3.12.: Processing statistics from MAD Excerpt was taken from XPREP documentation file. Underlined in green are the last figures before the anomalous signal-to-noise ratio drops below 1.3, at which point the resolution cutoff is recommended.

After preparing the peak dataset with XPREP SHELXD (Sheldrick, 2010) was run to find the heavy-atom positions. The number of molecules in the asymmetric unite was estimated with Bernhard Rupps Matthews probability calculator http://www. ruppweb.org/mattprob/default.html (Kantardjieff and Rupp, 2003, Matthews, 1968). The most probable number of molecules for the hetero-tetramer $\mathrm{DCTN1} 1_{412-533}-\mathrm{IC}_{2-47}$ was 4 with an overall probability of $54 \%$ and a solvent content of $46.5 \%$. Each hetero- 
tetramer contains 10 selenomethionine (SeMet) positions, which equals to 40 positions in total. A search for 40 SeMet positions was started and 56 heavy-atom positions were found by the programme of which only 15 seemed to be of significance. As can be seen in figure 3.13 there is a drop in the graph after peak number 15 , which indicates the last significant occupation site. A later examination of the heavy-atom positions in the electron density map confirmed this assumption.

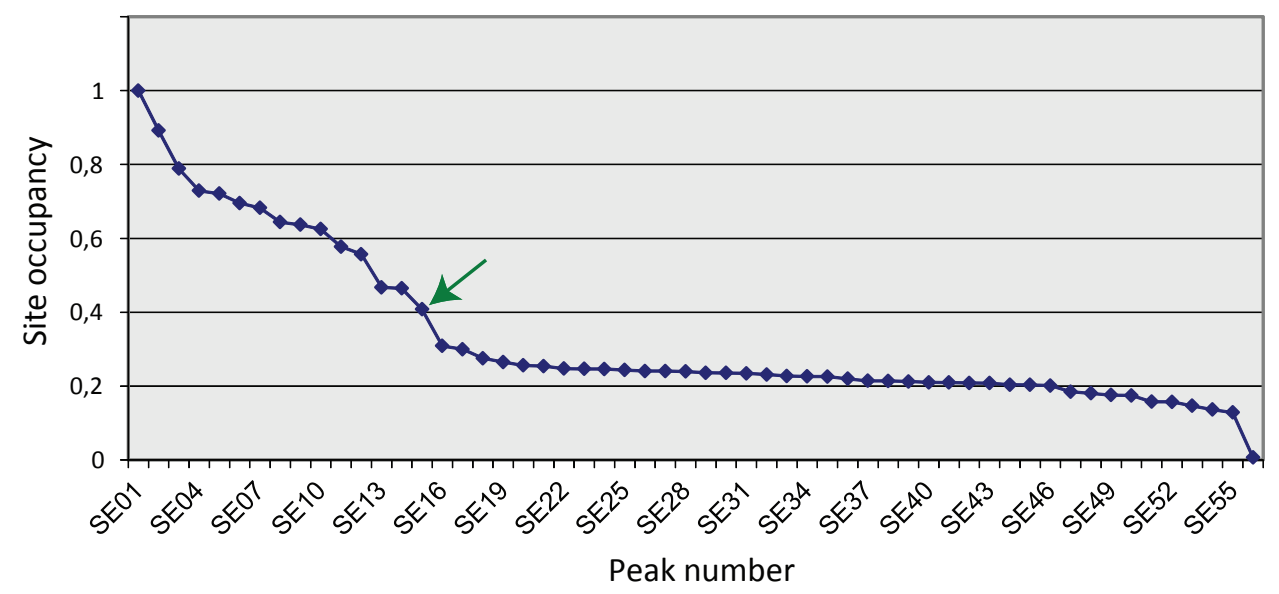

Figure 3.13.: Graph of the heavy atoms found in the peak dataset. The graph shows the heavy-atom occupancy versus the peak number. The green arrow indicates the last significant site of heavy-atoms.

Based on the afore calculated reference phases from heavy-atoms SHELXE estimated the electron density map of the complete macromolecule and improved the phases by density modification. Furthermore, it extended the phases to $3.4 \AA$ since the peak dataset showed good statistics up to this resolution. Because the systematic absence of reflections pointed to spacegroup $\mathrm{P} 4_{1} 2_{1} 2$ as well as its enantiomer $\mathrm{P} 4_{3} 2_{1} 2$ two maps (called 'original' and 'inverted', respectively) were calculated. As can be seen in figure 3.14 the 'original' map depicts the electron density of $\alpha$-helical structures whereas the 'inverted' map is not interpretable. Hence, $\mathrm{P} 4_{1} 2_{1} 2$ was chosen as spacegroup for the following steps. 

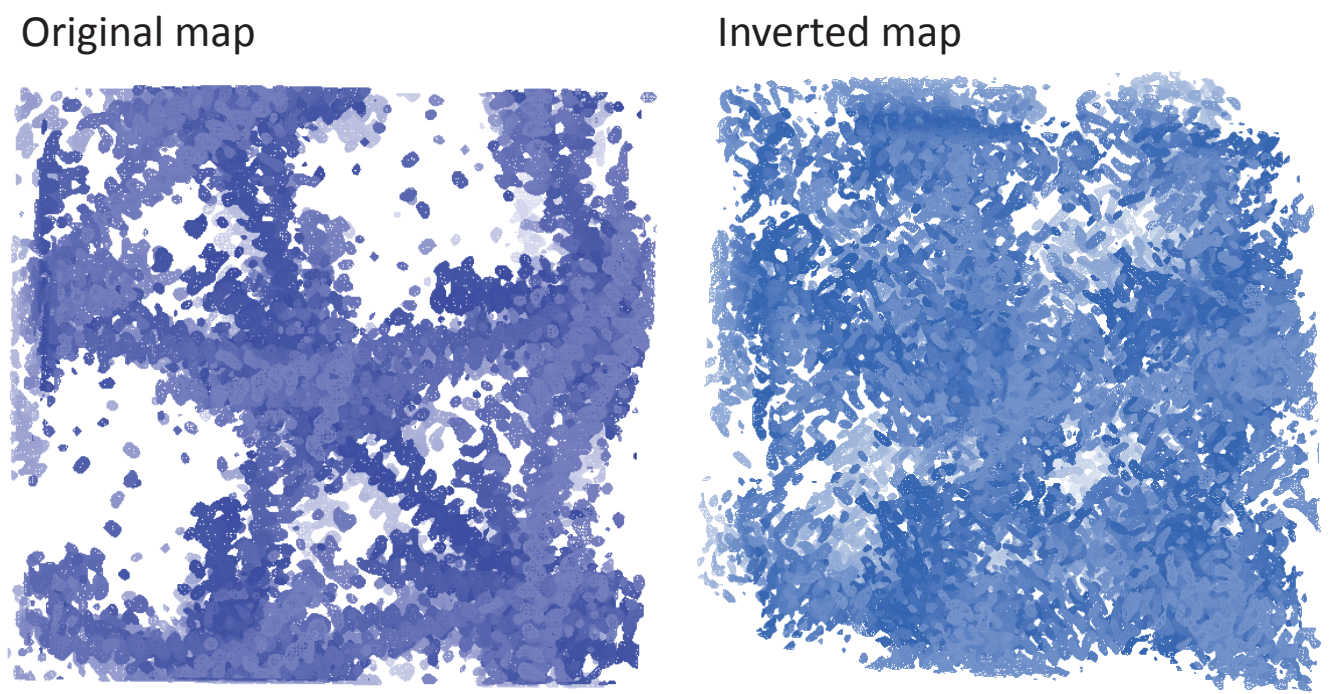

Figure 3.14.: Maps calculated by SHELX. (Left) Shown is the map calculated in spacegroup $\mathrm{P} 4_{1} 2{ }_{1} 2$. $\alpha$-helical structures are visible, which indicates that the map is correct (Right) This map was calculated based on spacegroup $\mathrm{P} 4_{3} 2_{1} 2$. There are no interpretable structural elements.

\subsection{Model building}

For modelling a structure into the electron density map the program $A C M I$ (DiMaio et al., 2007), which is specialized on low resolution modelling, was applied. The calculation of models was carried out in three sub-steps as described in subsection 2.10 .3 and took almost two weeks. Two runs were started with differing sequence inputs. On the one hand, the input file contained eight chains each of $\mathrm{DCTN1} 1_{412-533}$ and $\mathrm{IC}_{2-47}$, which is based on the aforementioned calculated Matthews probability (see section 3.4), which estimated that four molecules of the hetero-tetramer DCTN1 $1_{412-533}-\mathrm{IC}_{2-47}$ are in the asymmetric unit. On the other hand, only eight chains of DCTN1 $1_{412-533}$ were written in the second sequence input file. The second input file was based on the findings that a dissolved crystal applied on SDS gel only gave one band for DCTN1 ${ }_{412-533}$ while the $\mathrm{IC}_{2-47}$ band was almost invisible.

After the first two runs were finished two things became clear. One was that only DCTN1 $1_{412-533}$ amino acid sequences were built into the electron density map. And secondly, less than eight chains were built. Therefore, the results from the first run were dismissed and only the 20 built models from the DCTN1 $1_{412-533}$ run were inspected.

All models were checked in Coot (Emsley and Cowtan, 2004, Emsley et al., 2010) and

the longest $\alpha$-helical chains were copied into one pdb-file. Symmetry related atoms were visualized in Coot to ascertain the number of chains there are in the asymmetric 
unit 17. Based on this approach five $\alpha$-helices were found to fill the entire asymmetric unit. The chosen chains can be seen in figure 3.15 .

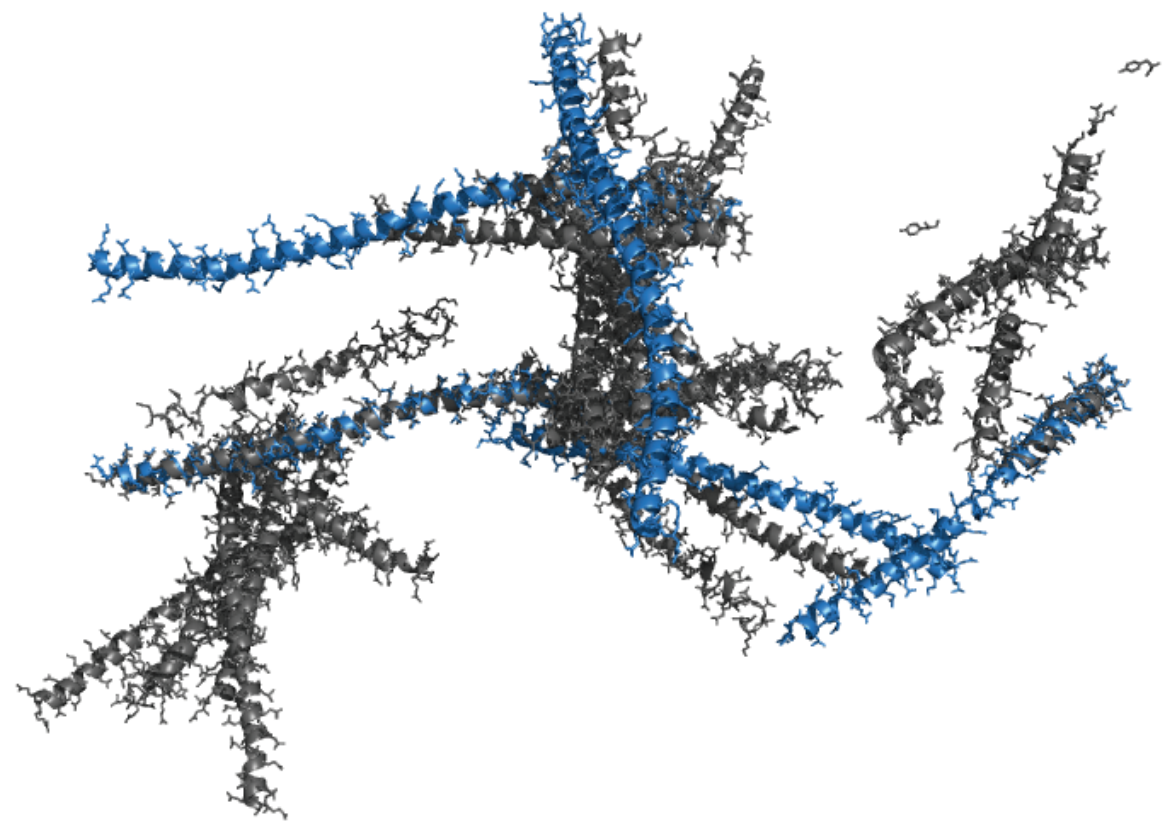

Figure 3.15.: Models calculated by $A C M I$ and the chains that were chosen for the initial model. The $\alpha$-helical chains, which are coloured in blue, were chosen as substructure for building the entire model.

These chains were consequently all moved into the electron density of the same asymmetric unit to simplify further work on the structure using shift_molecules in the software suite CNS (Brünger et al., 1998). Altogether, $67.5 \%$ of the five DCTN1 ${ }_{412-533^{-}}$ helices were built with $A C M I$. Four of these chains form two coiled-coils (chains A/B and $\mathrm{C} / \mathrm{D}$ ) while the fifth $\alpha$-helix forms a coiled-coil with its symmetry equivalent.

The chains were extended by using the model building tool Place helix here in Coot to fill the remaining electron density. The first 20 to 25 amino acids of DCTN1 $1412-533$ could not be built in since no electron density was visible. Also, during the first round of model building hardly any side-chain electron densities were visible. After a few rounds of refinement with Refmac (Version 5.7.0029) (CCP4, 1994, Murshudov et al., 2011) using restrained refinement, local NCS, and Babinet scaling for the resolution range 50-3.4 $\AA$ and subsequent model building the overall appearance of the $\alpha$-helices got more defined. The electron density for side-chains got more pronounced but no additional electron density, which could account for $\mathrm{IC}_{2-47}$ appeared. Several side-chains (especially at the $\mathrm{N}$ - and the C-terminus) could not be confidently placed in the elec-

\footnotetext{
${ }^{1}$ The asymmetric unit is the part of the unit cell that contains all the structural information and by applying symmetry operators reproduces the unit cell.
} 
tron density map. In particular, long side-chains as present in lysines or glutamic acids exhibited only partial electron densities. In addition, the fifth chain (E), which forms a coiled-coil with its symmetry equivalent (E'), exhibits chain breaks in the electron density between amino acids Arg479 and Glu491. In the final rounds of model building the MolProbity server (Chen et al., 2010) was used for validating the geometry of the structure. If applicable, changes were carried out on the structure. The final value of the residual factor $\left(\mathrm{R}_{\mathrm{work}}\right)$ decreased to $28.2 \%$ after an initial $33 \%$ after the first refinement round. The final value of the free residual factor $\left(R_{\text {free }}\right)$, which was calculated from $5 \%$ of the reflections that were not included into the refinement cycles, reached $30.6 \%$ (initially, 35\%).

\subsubsection{Molecular replacement with $\mathrm{DCTN1} 1_{412-533}-\mathrm{IC}_{2-47}$ dataset taken from crystal grown at $\mathrm{pH} 7.3$}

One dataset was taken from a crystal derived from $\mathrm{DCTN1} 1_{412-533}-\mathrm{IC}_{2-47}$ after dialysis using seeding. Other than the derivative and native DCTN1 $1_{412-533}-\mathrm{IC}_{2-47}$ crystals this crystal grew at pH 7.3 and diffracted to $3.4 \AA$. The dataset was processed with $X D S$ and displayed similar cell parameters like the derivative dataset $(\mathrm{a}=\mathrm{b}=156.82, \mathrm{c}=$ 133.08, $\alpha=\beta=\gamma=90^{\circ}$ ). Molecular replacement using Phaser MR (McCoy et al., 2007 ) implemented in the $C C P 4$ program suite and the DCTN1 $1_{412-533}$ coiled-coil structure as search model was performed to solve the phases. The resulting model was refined in Refmac. Electron density which could have accounted for the dynein IC did not appear near $\mathrm{DCTN1}_{412-533}$.

\subsubsection{Extending phases to new native dataset}

For a large number of native crystals no better diffraction than the one found in the derivative crystal dataset could be measured. Most crystals showed diffraction between 6 and $3.5 \AA$ and no additive screen or other construct could improve that. Finally, a native crystal diffracted until $2.9 \AA$ (see subsection 3.3 for crystallization condition and data processing statistics). The structure coordinates based on the $3.4 \AA$-dataset was refined in Refmac against the new $2.9 \AA$-dataset (resolution range 2.9-50 $\AA$ ) using TLS \& restrained refinement, local NCS and Babinet scaling. The new electron density map shows more features for side-chains, which can be seen in figure 3.16. Due to the better resolution of the map several side-chains could be placed with more confidence. However, most of the first 20 to 25 amino acids of the N-terminus were still not visible 
and no electron density for $\mathrm{IC}_{2-47}$ appeared. Also, the chain breaks in the map in the middle of chain E (Arg479 and Glu491) showed no improvement.
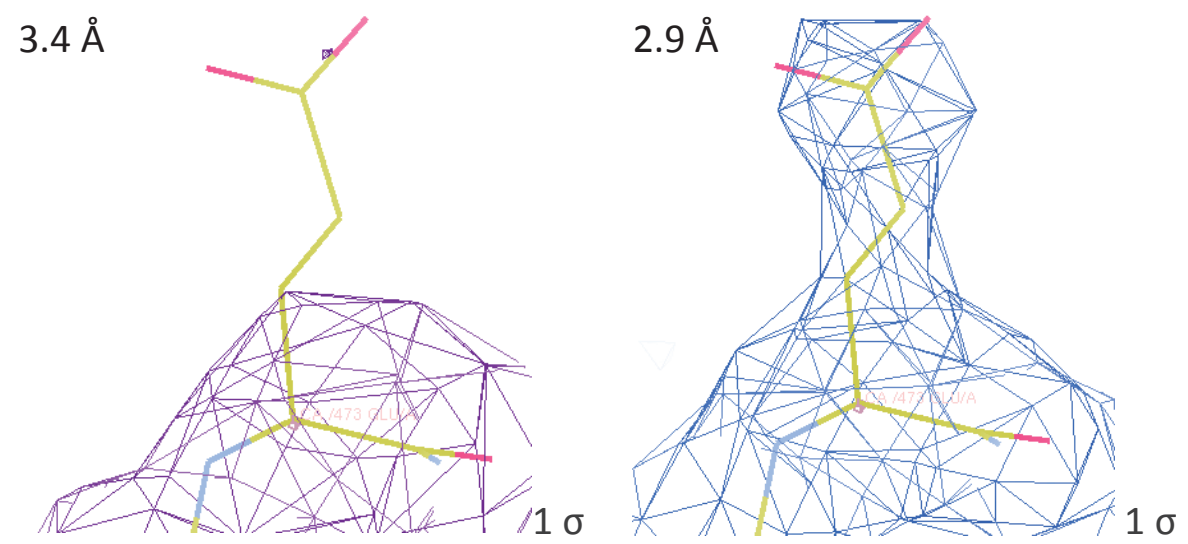

Figure 3.16.: Electron density map at 3.4 and $2.9 \AA$ Exemplarily for other amino acids, the electron density maps at 3.4 and $2.9 \AA$ of glutamic acid 473 is shown. Both maps are contoured at $1 \sigma$.

Subsequently, the structure was improved in Coot and refined in Refmac in several cycles. The final $R_{\text {work }}$ and $R_{\text {free }}$ values are 0.259 and 0.281 , respectively. An overview of the crystal data and refinement statistics can be found in section 3.1. The analysis of the structure was performed with MolProbity. It showed a good overall geometry with $98.57 \%$ of the amino acids in the favoured region of the Ramachandran plot and the rest in the allowed region.

Table 3.1.: Crystal data and refinement statistics

\begin{tabular}{lcc}
\hline & Derivative crystal & Native crystal \\
\hline Crystal data & & \\
Space group & $\mathrm{P} 4_{1} 2_{1} 2$ & $\mathrm{P} 4_{1} 2_{1} 2$ \\
Cell parameters & & \\
$\mathrm{a}, \mathrm{b}, \mathrm{c}[\AA]$ & $156.34,156.34,131.14$ & $155.32,155.32,129.26$ \\
$\alpha, \beta, \gamma\left[^{\circ}\right]$ & $90,90,90$ & $90,90,90$ \\
Resolution $[\AA]$ & $50-3.4(3.6-3.4)$ & $40-2.9(2.94-2.9)$ \\
Completeness of data $[\%]$ & $99.4(96.7)$ & $100(99.9)$ \\
Unique reflections & 42,432 & 35,585 \\
Multiplicity & $6.2(5.9)$ & $26.3(27.2)$ \\
$\mathrm{CC} 1 / 2$ & & $100(73.4)$ \\
$<\mathrm{I} / \sigma \mathrm{I}>$ & $8.21(1.87)$ & $25.37(1.78)$ \\
\end{tabular}


Table 3.1: Crystal data - continued from previous page

\begin{tabular}{|c|c|c|}
\hline & Derivative crystal & Native crystal \\
\hline \multicolumn{3}{|l|}{ Refinement statistics } \\
\hline $\mathrm{R}_{\text {work }}$ & & 0.259 \\
\hline$R_{\text {free }}$ & & 0.281 \\
\hline \multicolumn{3}{|l|}{ R.m.s.-deviations } \\
\hline Bond length $[\AA]$ & & 0.015 \\
\hline Bond angle $\left[^{\circ}\right]$ & & 1.616 \\
\hline Number of atoms & & 4102 \\
\hline Average B-factor $\left[\AA^{2}\right]$ & & 109.77 \\
\hline \multicolumn{3}{|l|}{ Ramachandran } \\
\hline in favoured regions [\%] & & 98.57 \\
\hline in allowed regions [\%] & & 1.43 \\
\hline
\end{tabular}

\subsection{Overview of the structural characteristics of the DCTN1 $1_{412-533}$ coiled-coil}

The final model consists of five $\alpha$-helical chains $(\mathrm{A} \rightarrow \mathrm{E})$ with a total of 488 amino acids and has a Matthews coefficient of $6.79 \AA^{3}$ /Dalton, which corresponds to a solvent content of $81.74 \%$. Water molecules were not placed into the model due to the low resolution. The five chains of DCTN1 $1_{412-533}$ start between amino acids Glu431 and Leu436 and end between Gln530 and Glu532. There was no electron density for the first 19-24 amino acids and the last 1-3 residues. Chains are between 94 and 100 amino acids in length, with chain E forming the shortest helix. Chains A/B, C/D, and E/E' each form a left handed parallel homodimeric coiled-coil as depicted in figure 3.17 A. Analyses with Socket (Walshaw and Woolfson, 2001) and Twister (Strelkov and Burkhard, 2002) were conducted to check whether the DCTN1 $1_{412-533}$ coiled-coils exhibit a continuous heptad motif typical for coiled-coil structures. No stutters or stammers that would result in local distortions of the geometry and that are implicated by interruptions in the heptad motif could be found. Both programs marked the corresponding amino acids with the same letter (abcdefg) of the heptad motif. All in all, each $\alpha$-helix consists of 14 continuous heptad motif repeats. Furthermore, Twister calculated that the average turn of the $\alpha$-helix depending on the coiled-coil consists of 3.60 to 3.63 residues and the $\alpha$-helical rise per residue is $1.51 \AA$ ( $1.52 \AA$ for $\left.\mathrm{E} / \mathrm{E}^{\prime}\right)$. The most pronounced difference 
between the three coiled-coils were found in the coiled-coil pitch 2 . Chains $\mathrm{C} / \mathrm{D}$ exhibit the smallest pitch with $149.9 \AA$ followed by coiled-coil A/B with $152.0 \AA$. Chains E/E' display the largest coiled-coil pitch with $158.5 \AA$.

As shown in figure $3.17 \mathrm{~B}$, the asymmetric unit is made up of five helices. All of them exhibit the same $\mathrm{N} \rightarrow \mathrm{C}$ direction. Coiled-coils $\mathrm{A} / \mathrm{B}$ and $\mathrm{C} / \mathrm{D}$ are positioned on each end of helix $\mathrm{E}$ in an obtuse angle $\left(130^{\circ}-150^{\circ}\right)$.

A

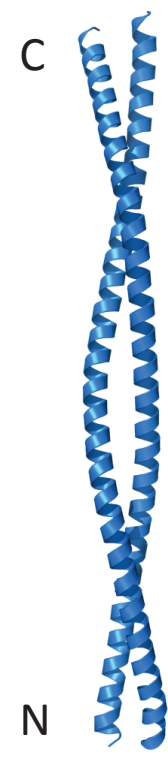

A B

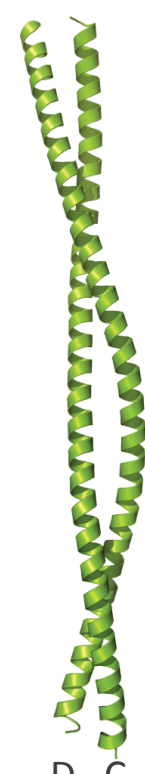

D C
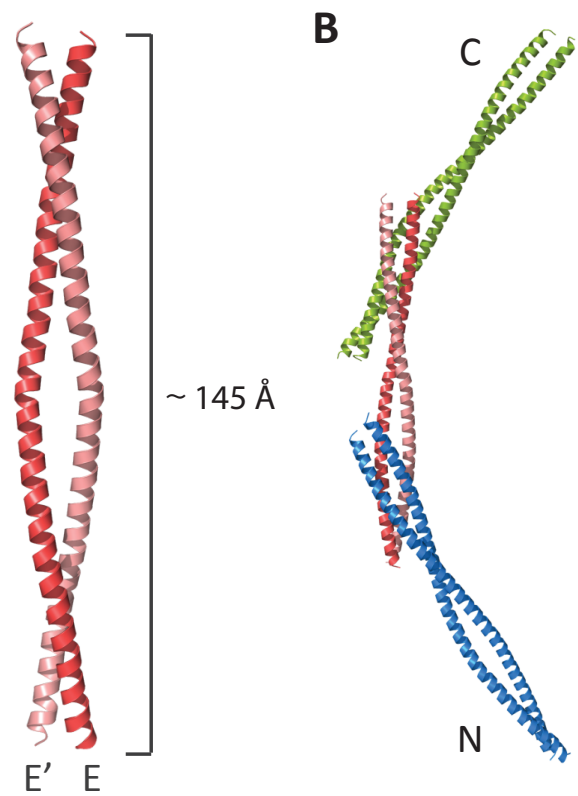

Figure 3.17.: Overview of the solved DCTN1 $1_{412-533}$ coiled-coil structure. (A) Shown are the three coiled-coils made up of chains A/B (blue), C/D (green) and $\mathrm{E}$ with its symmetry mate (E')(red). They have an average length of $145 \AA$. (B) The location of the coiled-coils in the asymmetric unit.

An analysis of the heptad positions in the structure (see appendix C.1 for a helical wheel representation) was carried out. The hydrophobic positions $a$ and $d$ also contain polar, uncharged amino acids like glutamine and asparagine as well as a charged glutamic acid (Glu475). A total of $28.5 \%$ non-hydrophobic amino acids occupy these positions. $59 \%$ of the preferentially charged positions $e$ and $g$ are populated by hydrophobic and polar, uncharged amino acids. Positions $b, c$, and $f$ can be occupied by any amino acid. In DCTN1 $1_{412-533}$ half of these positions are filled with negatively charged glutamic acid and aspartic acid, $14.8 \%$ are positively charged, $24 \%$ are hydrophobic residues and the rest is occupied with polar, uncharged amino acids.

${ }^{2}$ Coiled-coil pitch is the length of a coiled-coil in which it makes one complete turn. 


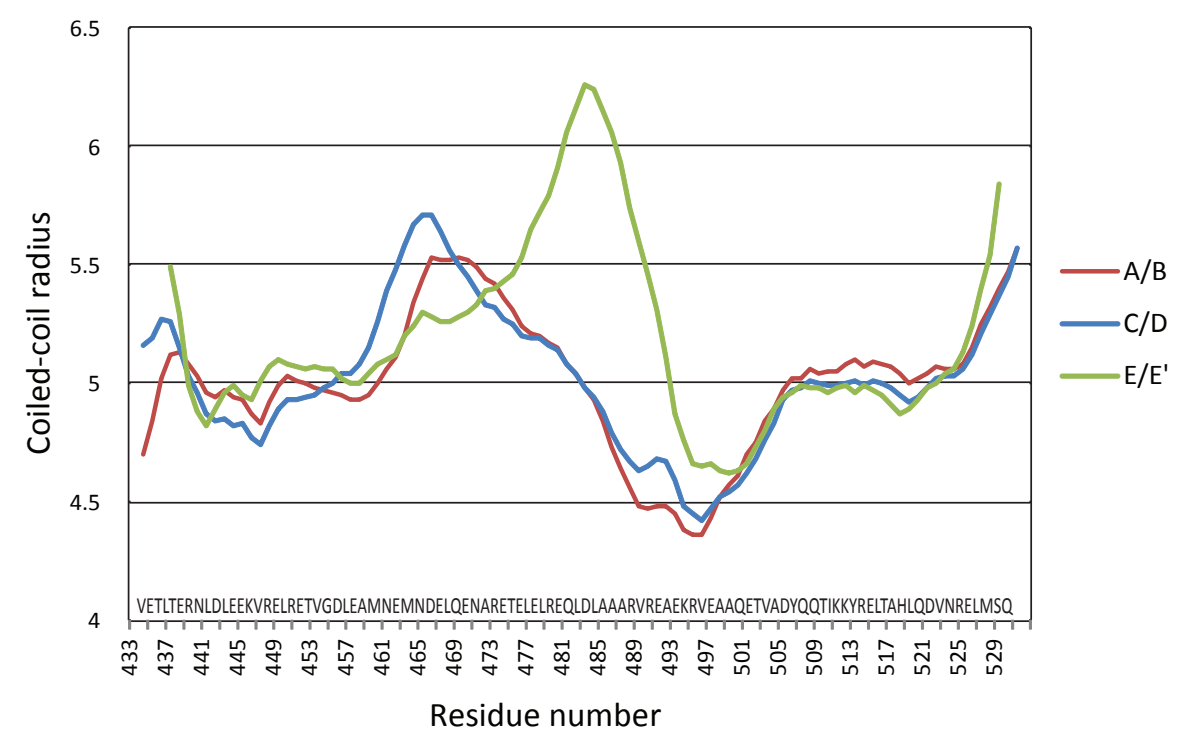

Figure 3.18.: Graphical representation of the coiled-coils radii. The radii of the three coiled-coils determined by Twister are depicted in dependence on the residue. Coiled-coils A/B, C/D and E/E' are coloured in red, blue, and green, respectively.

Furthermore, the coiled-coil radii calculated with Twister were examined. The distance between the helical axes in coiled-coils $\mathrm{A} / \mathrm{B}$ and $\mathrm{C} / \mathrm{D}$ range from $\approx 8.7$ to $11.4 \AA$ with a mean distance of $\approx 10 \AA$. Chains $\mathrm{E} / \mathrm{E}$ ' display a distance range of $\approx 9.2$ to $12.4 \AA$ and a mean distance of $10.2 \AA$. As can be seen in figure 3.18 the coiled-coil radii of $\mathrm{A} / \mathrm{B}$ and $\mathrm{C} / \mathrm{D}$ proceed similarly. An N-terminal stretch of four canonical leucines in $d$-positions (Leu436, 443, 450, 457) and mostly valines in $a$-position (Val433, Val447, Val454; Asn440) fluctuates slightly around the mean radius of $5 \AA$. A second area which displays mean radii is located at the C-terminus (residues 503-523) of the fragment and contains canonical hydrophobic as well as polar residues (Val503, Ile510, Thr517 in $a$ and Tyr506, Tyr513, Leu520 in $d$ ). There are also two distinct features in the graph that deviate from the mean radius. One is a peak with higher radii up to $5.7 \AA$ between residues 455 (459 for coiled-coil A/B) and 483. In this area there are non-hydrophobic residues in positions $a$ and $d$ (Asn461, Gln468, Glu475 in $a$ and Asn464 in $d$ ). This peak is followed by a drop in cc-radius $(4.4 \AA)$ between residues $483-505$. Here, position $d$ is occupied with a cluster of alanines (Ala485, Ala492, Ala499). E/E' on the other hand only roughly follows a similar radii course. Like the other coiled-coils there is a rise in cc-radius starting at residue 457 but the increase is shallower. However, instead of decreasing like in $\mathrm{A} / \mathrm{B}$ and $\mathrm{C} / \mathrm{D}$ the radii sharply increase until residue $483(6.3 \AA)$. It then drops to $4.7 \AA$ at residue 499 before rising again. There is also a higher radius at the C-terminus (> $5.5 \AA$ ), which can be found in every coiled-coil. 
All three coiled-coils exhibit a similar almost straight shape. The question arose how much they differ from each other. Therefore, the main-chain of the structures were superimposed using least square fit of residues Met432 to Glu465 in Coot. Chains C and E from coiled-coils C/D and E/E', respectively, were superimposed on helix A. They both gave low root-mean-square deviations (r.m.s.d.), 0.487 and $0.364 \AA$ for chains $\mathrm{C}$ and E, respectively. Superimposing residues 432 to 465 of Chain D to A led to a higher r.m.s.d. value $(1.13 \AA)$. Hence the superimposition of $\mathrm{C}$ to A was preferred. Since E' is the symmetry mate of helix E both superimpositions gave identical results.

Figure 3.19 shows the superimposition from the side and from the front (C-terminus). While coiled-coil E/E' lies in the middle, A/B and C/D each bend in opposite directions away from E/E'. Superimposing $3^{3}$ coiled-coils A/B and C/D on E/E' result in a maximum $\mathrm{C} \alpha$ distance of 23.93 and $19.11 \AA$, respectively. The maximum distance of $\mathrm{C} / \mathrm{D}$ to $\mathrm{A} / \mathrm{B}$ is $35.26 \AA$. The minimum and average distances can be seen in table 3.2 . Superimposing the main-chain of the whole coiled-coils resulted in low r.m.s.d values (see table 3.2 ).

Table 3.2.: Superimposition

\begin{tabular}{lcccc}
\hline & \multicolumn{2}{c}{ Distance $[\AA]$} & r.m.s.d \\
& minimum & maximum & average & whole main-chain \\
\hline $\mathrm{C} / \mathrm{D} \rightarrow \mathrm{A} / \mathrm{B}$ & 1.38 & 35.26 & 13.98 & 2.36 \\
\hline $\mathrm{A} / \mathrm{B} \rightarrow \mathrm{E} / \mathrm{E}^{\prime}$ & 1.47 & 23.93 & 11.42 & 1.28 \\
\hline $\mathrm{C} / \mathrm{D} \rightarrow \mathrm{E} / \mathrm{E}^{\prime}$ & 0.09 & 19.11 & 6.33 & 2.17
\end{tabular}

In order to analyse the bending of $\mathrm{A} / \mathrm{B}$ and $\mathrm{C} / \mathrm{D}$ the programme $C C B E N D S(\overline{B r o w n}$, 2010) was employed. Only those residues at which the size of the local axis bend was a peak and greater than $3^{\circ}$ were considered. For coiled-coil A/B a $3.9^{\circ}$ and a $4.8^{\circ}$ bend was found at residue Leu482 and Ala504, respectively. The direction of bend is $-8.6^{\circ}$ for the first and $-166.2^{\circ}$ for the second residue. Coiled-coil $\mathrm{C} / \mathrm{D}$ contained bends at similar positions. At residue Leu482 a $5.1^{\circ}$ and at Asp505 a $6^{\circ}$ bend was detected which display a direction of bend of $7.5^{\circ}$ and $-143.2^{\circ}$, respectively. No bends were found in coiled-coil E/E'.

\footnotetext{
${ }^{3}$ This was done in PyMol using the Phyton script color_by_rmsd by Shivender Shandilya, Jason Vertrees, and Thomas Holder.
} 

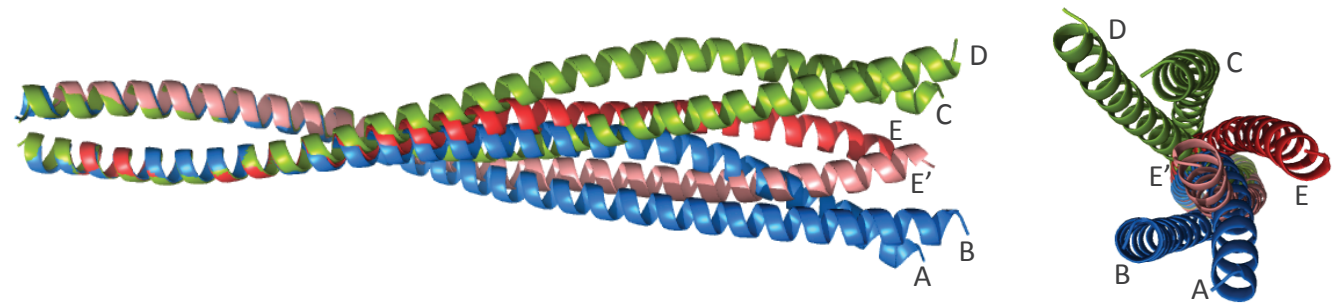

Figure 3.19.: Superimposition of the three coiled-coils. Residues 432 to 465 of all three coiled-coils were superimposed in Coot to see differences in the overall shape. (Left) View of the superimposition from the side and (Right) from the front of the C-terminus.

Furthermore, B-values, which are a measure of residue flexibility 4 , were mapped on the structure. The $\mathrm{C}_{\alpha}$ B-factors and the residue B-factors vary between 50 and $220 \AA^{2}$ and between 40 to $240 \AA^{2}$, respectively. Since several side-chains are only insufficiently supported by electron density only $\mathrm{C}_{\alpha}$ B-factors were considered. All helices feature regions at both termini with lower B-factors as depicted in figure $3.20 \mathrm{~A}$ in blue. They correspond to crystal contacts. The middle and the ends of all helices show higher B-values depicted in green to red. The areas coloured in yellow to red coincide with poorer electron density partly resulting in chain-breaks. In contrast to the other two coiled-coils, E/E' exhibits very high B-values in the middle. This can also be seen in graph 3.20 B where helix E shows an overall higher B-value between residues 466 to 495 5. All helices show a decrease of main-chain B-values around residues 452 to 462 and 505 to 515 , except for chain B, which exhibits hardly any decrease in the second region.

The two crystal contact regions with lower B-values contain polar contacts ${ }^{6}$ between coiled-coils in these areas. These contacts are visualized in figure 3.21. Most of them are hydrogen bonds that are characterized by residues that donate their covalently bound hydrogen to a negatively charged acceptor atom. Examples are hydrogen bonds formed between Asp456/E and Tyr513/B, Glu445/E and Gln507/A, and Tyr506/E and Glu452/D. Furthermore, Glu501/E forms a salt bridge with Arg451/C which occurs between amino acids of opposite electron charges. In general, one can say that there are polar contacts between the C-terminal region of chains $\mathrm{A}$ and $\mathrm{B}$ with the N-terminal area of chain E. The N-terminal regions of chains $\mathrm{C}$ and D are in contact with the C-terminal area of helix E. Likewise, there are polar contacts between the

\footnotetext{
${ }^{4}$ Low B-values indicate well order regions while higher B-values are a sign of high flexibility or disorder.

${ }^{5} \mathrm{~B}$-factor values of the main-chain were extracted from the pdb-file using the program Baverage in $\mathrm{CCP}_{4}(\mathrm{CCP} 4,1994)$.

${ }^{6}$ Polar contacts involving side-chains were calculated in PyMol.
} 
A
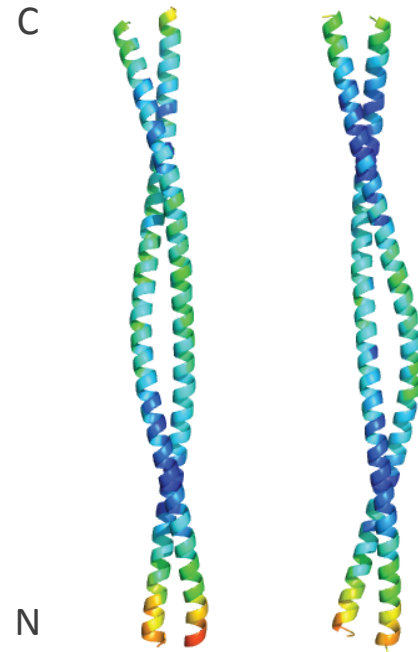

A B

D C

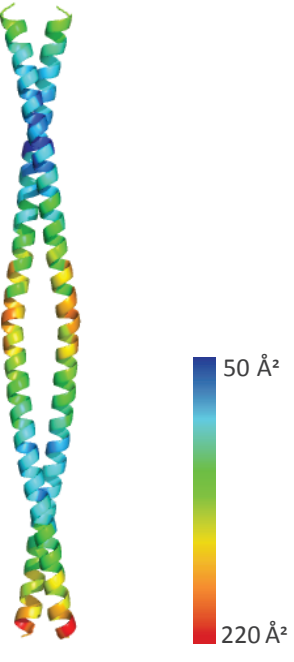

B

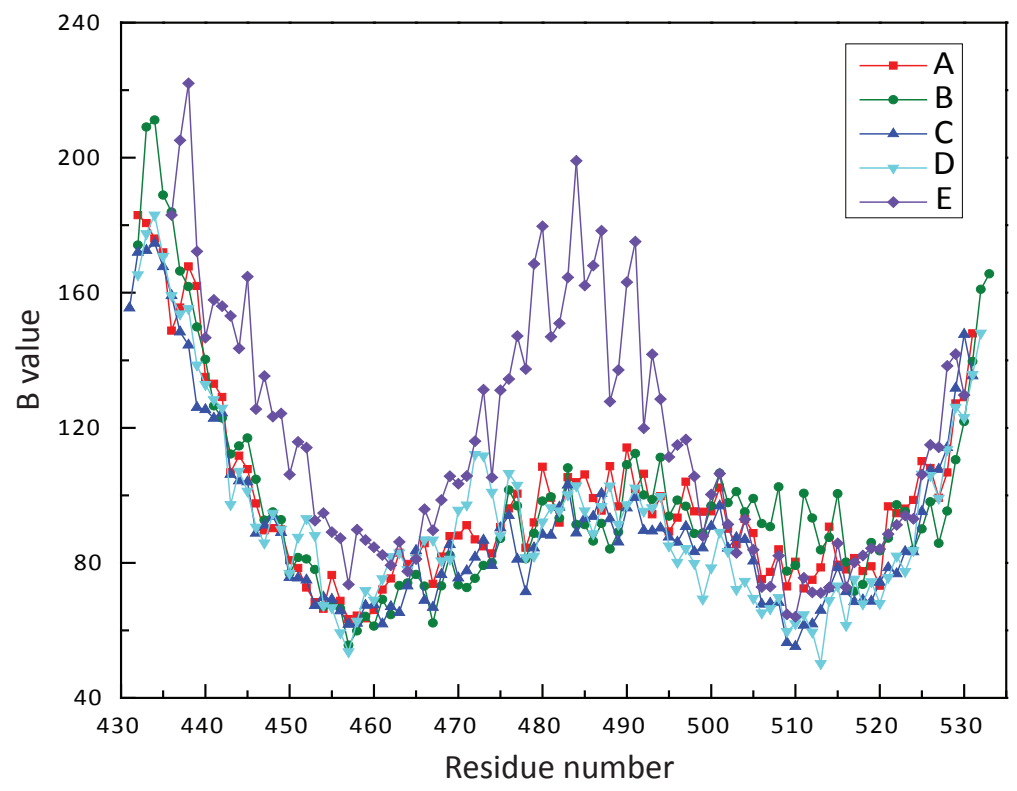

Figure 3.20.: Representation of B-factors. (A) $\mathrm{C}_{\alpha}$ B-factors are mapped on the structure. Each coiled-coil shows areas of lower (blue to green) and higher flexibility (yellow to red). In contrast to coiled-coils A/B and C/D, E/E' shows very high flexibility in the middle. (B) Graphical representation of main-chain B-factors of the five helices against the residue numbers.

C-terminal regions of coiled-coil C/D and the N-terminal area of chains A and B with symmetry mates.

Hydrogen bonds can be found throughout the structure. This is especially true for the single helices because they are stabilized by hydrogen bonds. The $\mathrm{C}=\mathrm{O}$ group of an amino acid forms a hydrogen bond with the N-H group of the amino acids four residues apart. There are a few intra as well as inter side-chain side-chain polar contacts. For example, both Asn524 in chains A and B form a hydrogen bond as well as in 
chains C and D. Salt bridges are found between Arg488/C and Glu493/D and between Arg488/A and Glu493/B (shown in figure 3.22). All in all, there are about eight polar intramolecular contacts in each coiled-coil. However, if the cut-off for polar contacts is reduced from $3.6 \AA$ (default in PyMol) to $3.2 \AA$ only one contact each remains. In the case of coiled-coil A/B it is a hydrogen bond between Asn461-Asn461 and coiled-coil C/D still has a salt bridge between Arg488/C and Glu493/D. If the cut-off is further reduced to $3.0 \AA$ no interaction remains between the helices. Interestingly, polar contacts between coiled-coils that form the crystal contacts are slightly diminished in number but still a total of seven contacts remain. It appears, that on average, inter-coiled-coil polar contacts are stronger than intra-coiled-coil bonds.

In a further step to examine the structure the electrostatic surface of the coiled-coil was generated using PyMol. Based on the calculated pI (pI: 4.4) is was expected that DCTN1 $1_{412-533}$ would display an overall negatively charged electrostatic surface. As can be seen in figure 3.23 the N-terminal region displays a large elongated band of negative charge from Glu434 to Asp483. Two further negatively charged patches can be found around Glu501 and Glu532. Positively charged areas can be seen around Arg451, Lys494, Lys511 to Arg514, and Arg525. Altogether it can be said that the molecule is indeed predominantly negatively charged.

In order to see if this negatively charged area is only found in chicken DCTN1 $1_{412-533}$ or if this is typical for all DCTN1 proteins a weblogo (Crooks et al., 2004, Schneider and Stephens, 1990) 7 of a DCTN1 alignment containing 236 sequences was created. In figure $3.23 \mathrm{~B}$ the whole $\mathrm{DCTN} 1_{412-533}$ weblogo with the corresponding alignment positions is depicted. The area representing the solved structure is coloured in light grey. The asterisks in the image highlight conserved hydrophobic residues, which are found in positions $a$ and $d$ of the heptad motif. The weblogo shows a well conserved N-terminus of DCTN1 $1_{412-533}$ up to alignment position 952 (Asp483 in chicken DCTN1 $1_{412-533}$ ) containing many conserved acidic amino acids. This corresponds to the surface area that displays an elongated negatively charged band. Compared to the aforementioned region the following area mainly depicts less residue conservation. Exceptions are for example some conserved acidic amino acids at positions 960 (Glu491), 970 (Glu501), and 974 (Asp505) and alkaline residues at positions 981 (Lys512) and 983 (Arg514). Furthermore, threonine (Thr509) at alignment position 978 seems to be of great importance since it is highly conserved. A look into the polar contacts of the DCTN1 $1_{412-533}$ structure reveal a potential intramolecular hydrogen bond between Thr509 and the backbone oxygen of Asp505, which is also conserved within the eukaryotes.

\footnotetext{
${ }^{7}$ Created with WebLogo 3 using the webserver http://weblogo.threeplusone.com
} 
A

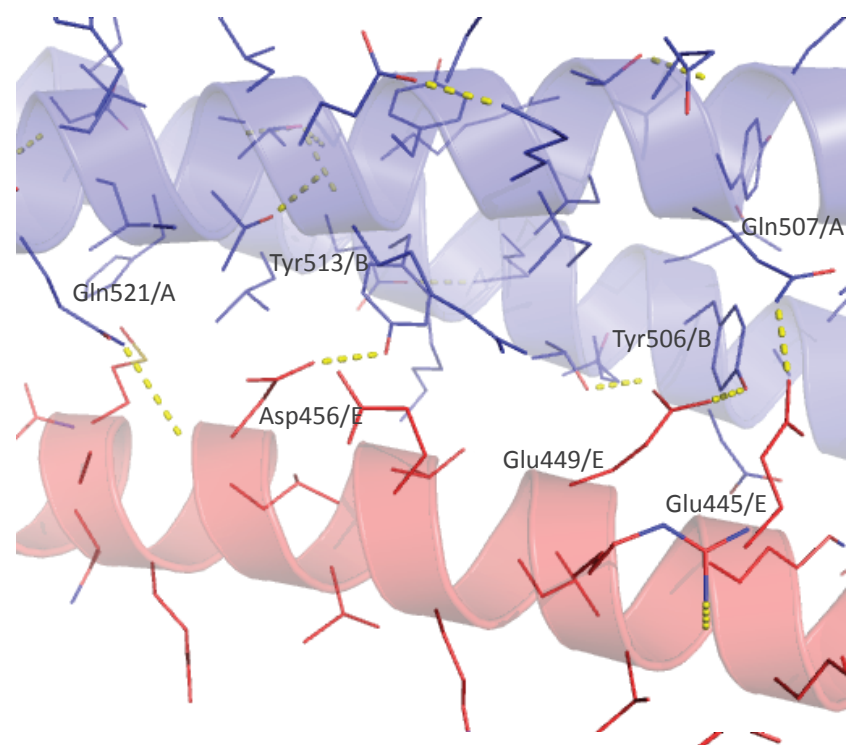

B

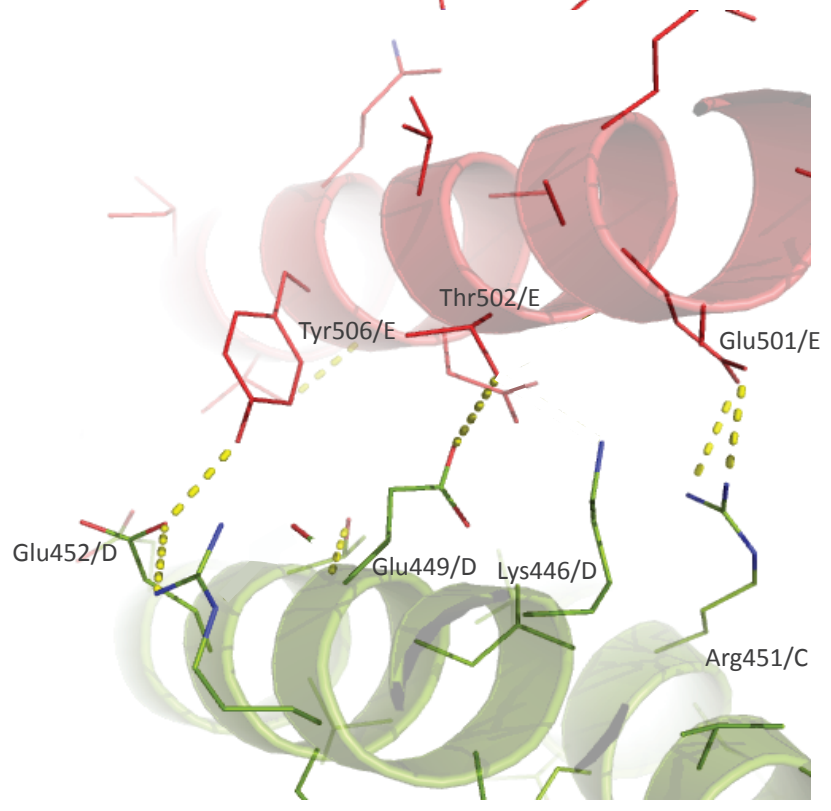

Figure 3.21.: Polar contacts between coiled-coils. Polar contacts are indicated by yellow dashed lines. (A) Hydrogen bonds between coiled-coil A/B and helix E are shown. (B) Hydrogen bonds and a salt bridge (Glu501/E and Arg452/C) found between coiled-coil C/D and helix E are depicted.

\subsection{Characteristics of dynein intermediate chain}

In contrast to $\mathrm{DCTN1} 1_{412-533}, \mathrm{IC}_{2-69}$ has a calculated $\mathrm{pI}$ of $9.3\left(\mathrm{IC}_{2-47}: \mathrm{pI}=9.7, \mathrm{IC}_{2-84}\right.$ : $\mathrm{pI}=9.2$ ). It has therefore a charge opposed to DCTN1 $1_{412-533}$ suggesting an electrostatic interaction between those two molecules.

The N-terminal region of IC is characterized by many alkaline amino acids. This is not 
A

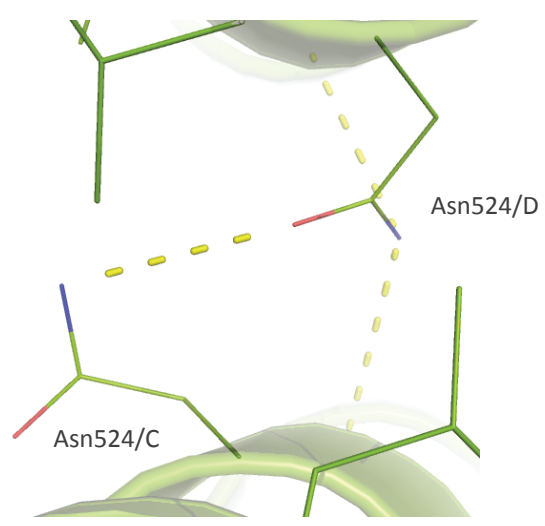

B

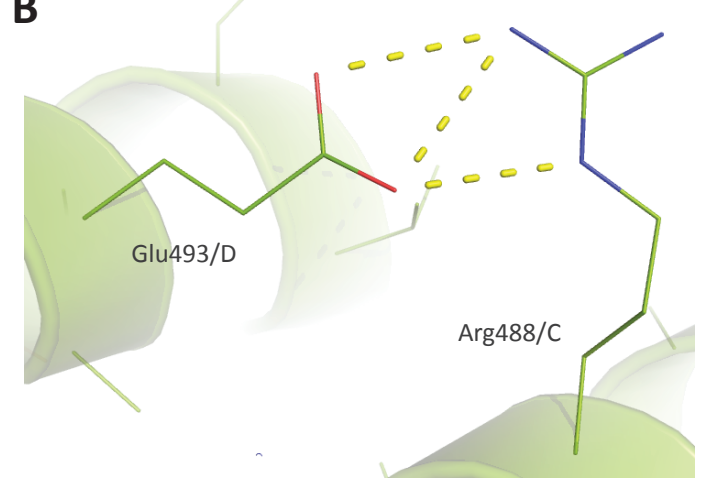

Figure 3.22.: Polar contacts within coiled-coils C/D. These polar contacts can also be found in coiled-coil A/B. Polar contacts are indicated by yellow dashed lines. (A) Hydrogen bonds between asparagines. (B) Salt bridge between $\operatorname{Arg} 488$ and Glu493.

just the case for human IC but can be found throughout the eukaryotes. A weblogo was created to illustrate the conservation of amino acid positions in the N-terminus of IC. It consists of 175 cytoplasmic IC sequences from 142 species which range from fungi, frogs, birds to vertebrates. Figure 3.24 A shows the weblogo of the first 122 alignment positions as well as the amino acid sequence of Drosophila melanogaster and of the two human cytoplasmic IC isoforms. Two conserved regions (alignment positions 8-42 and 62-86) indicated by high, wide stacks are apparent. They are separated by a less conserved region, which is illustrated by smaller stacks. Especially, the first region contains many conserved arginines, lysins as well as conserved leucines and glutamic acids which can also be seen by comparing the human and D. melanogaster sequences. The second region also contains alkaline and acidic amino acids and some moderately conserved glycine and proline positions. Towards the C-terminus of the sequence section several more proline and glycine positions show some degree of conservation. These amino acids are known to disrupt $\alpha$-helical structures. Accordingly, it would be expected that the first 69 amino acids of IC bind to the negatively charged regions of $\mathrm{DCTN}_{412-533}$.

Of interest are the alternatively spliced exons in the N-terminal regions of the two isoforms of human IC localized upstream of the two DCTN1 binding regions. They are conserved among other species like chicken, dog, and the pufferfish Takifugu rubripes. They have previously been reported in mice IC and showed tissue-specific expressions Kuta et al. (2010). Both human isoforms contain differentially included exons starting after amino acid 74 and 75 in IC1 and IC2, respectively (see figure 3.24 B). Both contain a differentially included exon (coloured in green), which is similar in length (17 aa 
A

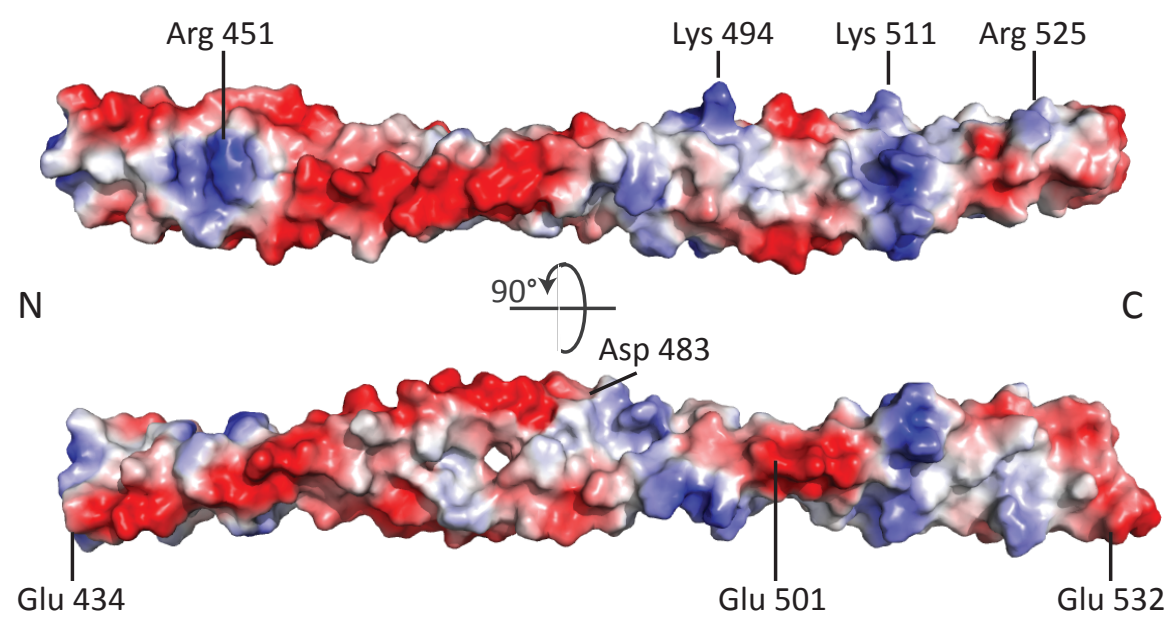

B

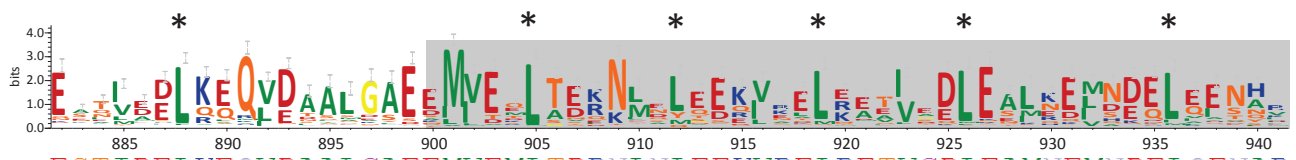

Hs ESTIDELKEOVDAALGAEEMVEMLTDRNLNLEEKVRELRETVGDLEAMNEMNDELOENAR Gg EKTVDELKEQVDAALGAEEMVETLTERNLDLEEKVRELRETVGDLEAMNEMNDELQENAR Dm EAIVADLQEQVDAALGAEEMVEQLAEKKMELEDKVKLLEEEIAQLEALEEVHEQLVESNH

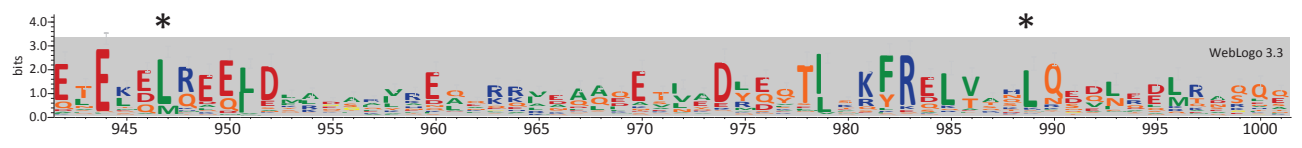

Hs ETELELREQLDMAGARVREAQKRVEAAQETVADYQQTIKKYRQLTAHLQDVNRELTNQQE Gg ETELELREQLDLAAARVREAEKRVEAAQETVADYQQTIKKYRELTAHLQDVNRELMSQQE Dm ELELDLREELDLANGAKKEVLRERDAAIETIYDRDQTIVKERELVQKLNDQLTELRDRNS

Figure 3.23.: Electrostatic surface charge and sequence conservation of DCTN1 $1_{412-533}$ coiled-coil. (A) The electrostatic surface was calculated in PyMol. Red areas represent negatively charged areas whereas blue depicts positive surface patches. The image below shows the molecule rotated by $90^{\circ}$ around the x-axis. (B) This weblogo of $\mathrm{DCTN1}_{412-533}$ illustrates the sequence conservation of 236 protein sequences. The overall stack height indicates the sequence conservation at that position while the height of the single amino acids at the position show the relative frequencies of the symbols. The width illustrates valid symbols at that positions (many gaps lead to thinner stacks). The N-terminus is well conserved up to position 952, which is equivalent to chicken Asp483. The C-terminus is less well conserved. However, there are some acidic and alkaline residues that show high conservation. The asterisks show conserved positions that correspond to $a$ and $d$ of the heptad motif.

and 18 aa in $\mathrm{IC} 1$ and IC2, respectively) and in sequence. For this exon an alternative 3' splice site is used leading to an extended boundary of the downstream exon. In front of this differentially included exon IC2 also contains a 7 aa long exon (orange), which is subject to exon skipping. Both differentially included exons lead to differing lengths (from 0 to $17 / 18$ to 25 aa) between two proline-containing sequence sections. 


\section{A}

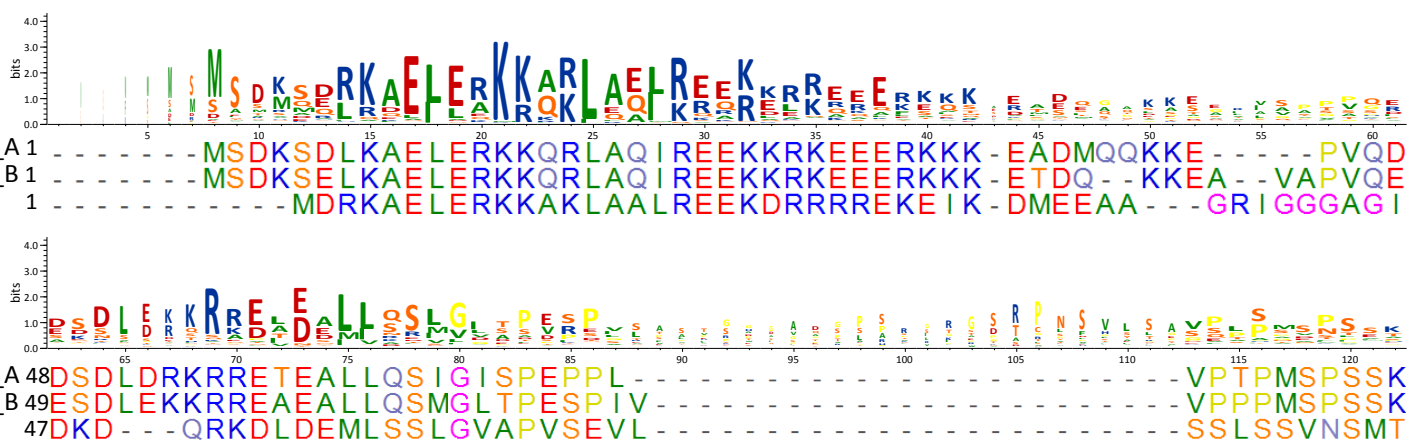

B Dynein intermediate chain 1

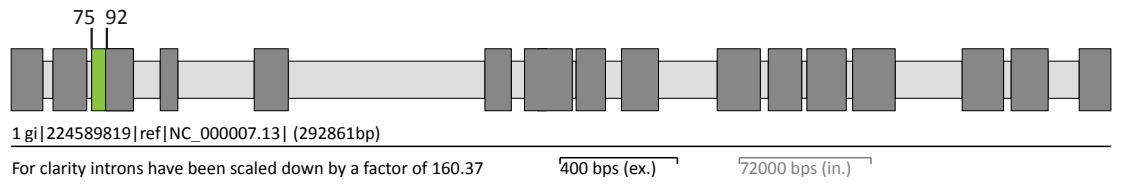

Dynein intermediate chain 2

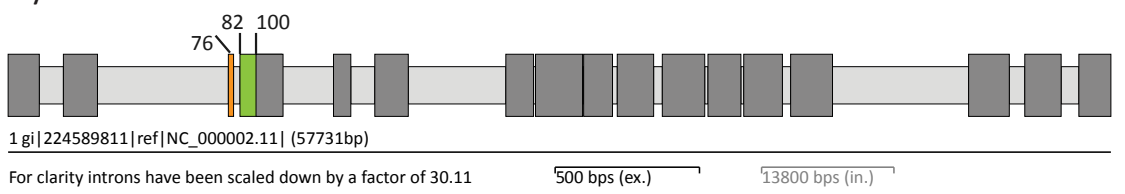

Figure 3.24.: Sequence analysis of IC. (A) The weblogo shows the IC sequence conservation of the N-terminal region. For the calculation of the weblogo 175 sequences from 142 species were used. The overall stack height indicates the sequence conservation at that position while the height of the single amino acids at this position show the relative frequencies of the symbols. The width illustrates valid symbols at that position (many gaps lead to thinner stacks). Altogether, there are two conserved regions. Underneath the logo are the sequences of human IC ( $H s$, isoforms A and B) and of Drosophila melanogaster $(D m)$. (B) The two isoforms of human cytoplasmic IC with their alternatively spliced exons in the N-terminal region were created with WebScipio (Hatje et al., 2013). Introns are illustrated in light grey and exons in dark grey. The alternatively spliced exons are coloured in green and orange. The green exons in both isoforms have a similar sequence and they are both spliced by an alternative 3' splice junction. The numbers indicate the respective start position of the exon in the amino acid sequence.

Secondary structure prediction tools like Marcoil (Delorenzi and Speed, 2002) and Logicoil (Vincent et al., 2013) all predict a coiled-coil structure for the first 44 to 67 amino acids of the N-terminal IC. Examining the amino acid sequence of IC gves rise to the question if it really forms coiled-coils since it does not feature a typical heptad motif. It is not uncommon for prediction tools to predict charged single $\alpha$-helices as coiled-coils (Peckham and Knight, 2009, Süveges et al., 2009).

A web tool, called WaggaWagga (http://www.motorproteine.de/waggawagga, Do- 
minic Simm), examines protein sequences for their propensity to form charged single $\alpha$-helices (CSAH) instead of coiled-coils. CSAH are characterized by a lack of a hydrophobic seam caused by charged amino acids in positions $a$ and $d$ of the heptad motif (Peckham and Knight, 2009). Coiled-coils on the other hand contain 75-80\% apolar amino acids in these positions (Conway and Parry, 1990) leading to the formation of the hydrophobic seam.

The result for $\mathrm{IC}_{1-69}$ derived from the WaggaWagga web application can be seen as a so-called heptad net in the top of figure 3.25. It shows that positions $a$ and $d$ while containing some hydrophobic amino acids are mainly filled by charged amino acids. This leads to many strong and weak interactions between oppositely charged amino acids, which are known to stabilize CSAH. It seems unlikely that a hydrophobic seam can develop under these conditions. In contrast to IC, DCTN1 $1_{412-533}$ features apolar amino acids in positions $a$ and $d$ (see figure 3.25 bottom). Except for one glutamic acid in position $a$ (Glu475), all charged residues are found in the remaining heptad positions. There are only a few predicted interactions between charged residues within the $\alpha$-helix. Furthermore, the overall percentage of charged residues is higher in $\mathrm{IC}_{1-69}$. In $\mathrm{DCTN} 1_{412-533} 41.3 \%$ of all residues are charged (R, K, D, and E) whereas in the putative single $\alpha$-helix $\mathrm{IC}_{1-69} 56.4 \%$ are charged. Taking these findings together, it is possible that the two ICs of dynein form single $\alpha$-helices instead of a coiled-coil.

\subsection{Chemical cross-linking of DCTN1 $142-533-\mathrm{IC}_{2-47}$ and $\mathrm{DCTN1}_{412-533}-\mathrm{IC}_{2-69}$}

Although dynein IC was part of the purified complex and hence part of the crystallization set up it was not present in the electron density map. Therefore, chemical cross-linking in combination with mass spectrometry was chosen to find out where IC interacts with DCTN1.

For the reaction the crosslinker $\mathrm{BS}^{3}$ was selected. It contains two reactive groups $(\mathrm{N}-$ hydroxysulfosuccinimide ester) at each end of a $11.4 \AA$ spacer arm. The ester groups react with amino groups found in lysines and at the N-terminus at $\mathrm{pH}$ 7-9 to form stable interactions. Due to the length of the spacer long distances between the interacting proteins can be bridged.

Two constructs, DCTN1 $1_{412-533}-\mathrm{IC}_{2-47}$ and DCTN1 $1_{412-522}-\mathrm{IC}_{2-69}$, were used for this experiment. Each $\mathrm{IC}_{2-47}$ molecule contains 12 lysine positions and each $\mathrm{IC}_{2-69}$ protein 13 lysine positions as potential cross-linking sites. The DCTN1 $1_{412-533}$ monomer features only six lysines. At first, 70-100 pmol of the protein complex were cross-linked with 


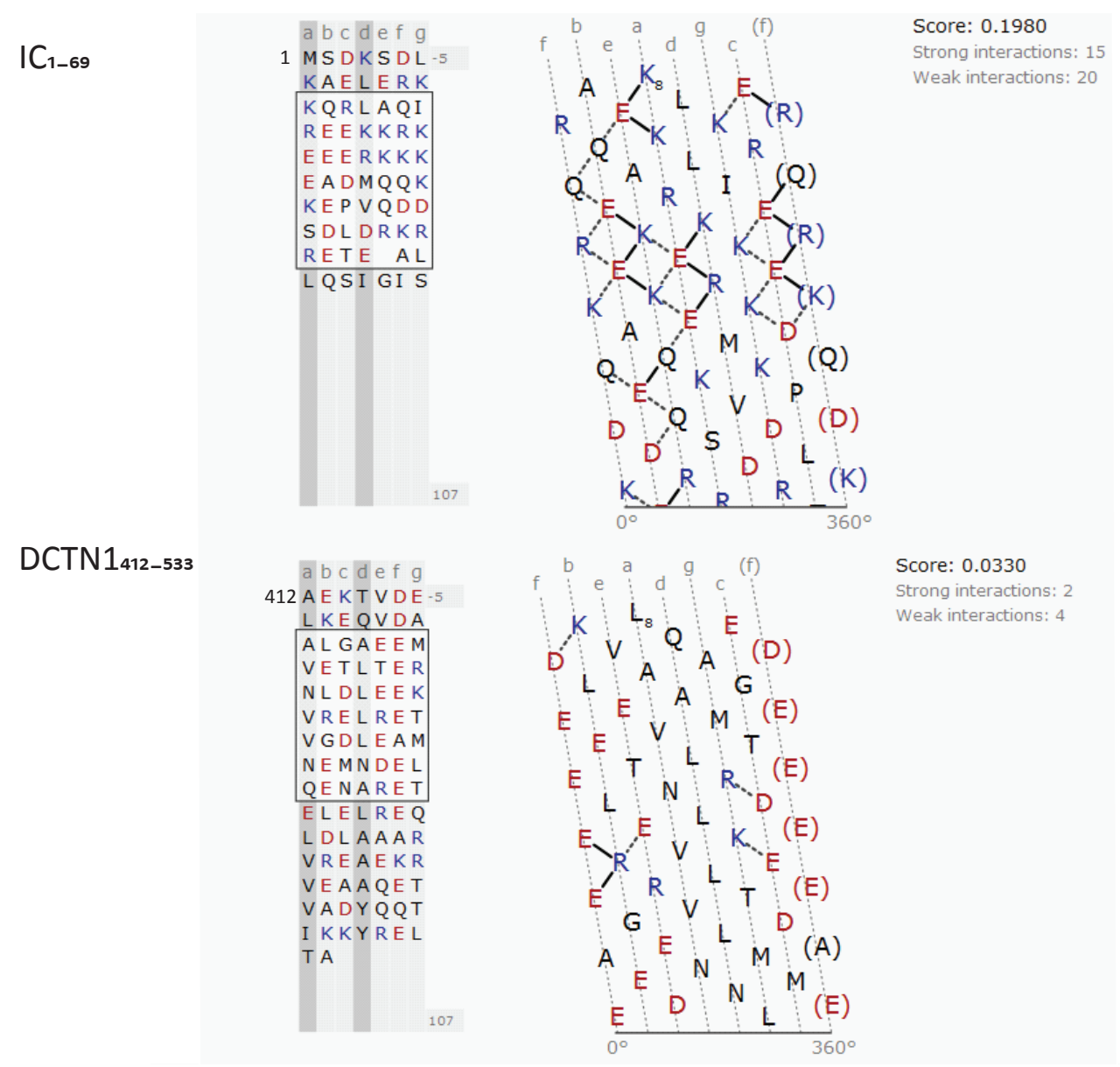

Figure 3.25.: WaggaWagga results for $\mathrm{IC}_{1-69}$ and $\mathrm{DCTN1} 1_{412-522}$. On the left side the amino acid sequence with their corresponding heptad position is shown. On the other side a heptad net is depicted to give a 2-D representation of the helix by opening up along a $\alpha$-helical seam and flattening it out. Thereby, the hydrophobic seam formed by $a$ and $d$ is located in the middle of the net. Charged residues are coloured in red (E, D) and blue (R, K). (Top) $\mathrm{IC}_{1-69}$ shows many charged residues in positions $a$ and $d$ and several interactions between these amino acids. Since there are hardly any apolar residues in these positions the formation of a hydrophobic seam seems unlikely. Therefore, a CSAH could be a possibility. (Bottom) The coiled-coil DCTN1 $1_{412-522}$ shows a distinct hydrophobic seam caused by apolar residues in positions $a$ and $d$ and only a few interactions.

increasing amounts of $\mathrm{BS}^{3}(0,25,62.5,125,250,500$, and $1000 \mu \mathrm{M})$ to determine which concentration was sufficient for a good cross-linking result without producing unspecific links. 125 and $250 \mu \mathrm{M}$ produced the best results. So all subsequent experiments were performed with these concentrations. All in all, DCTN1 $1_{412-533}-\mathrm{IC}_{2-47}$ protein was used for two experiments (125 and $\left.250 \mu \mathrm{M} \mathrm{BS}^{3}\right)$ and DCTN1 $1_{412-533}-\mathrm{IC}_{2-69}$ for four experiments (two each with 125 and $250 \mu \mathrm{M} \mathrm{BS}{ }^{3}$ ) resulting in a total of six replicas. Samples were prepared as described in section 2.11. The appropriate bands 
corresponding to DCTN1 $1_{412-533}$ cross-linked with IC (see figure 3.26) were cut from the gel. They were then digested with trypsin and the resulting peptides were extracted from the gel pieces and analysed via mass spectrometry as described in section 2.11. The search for cross-links was performed with pLink (Yang et al., 2012).

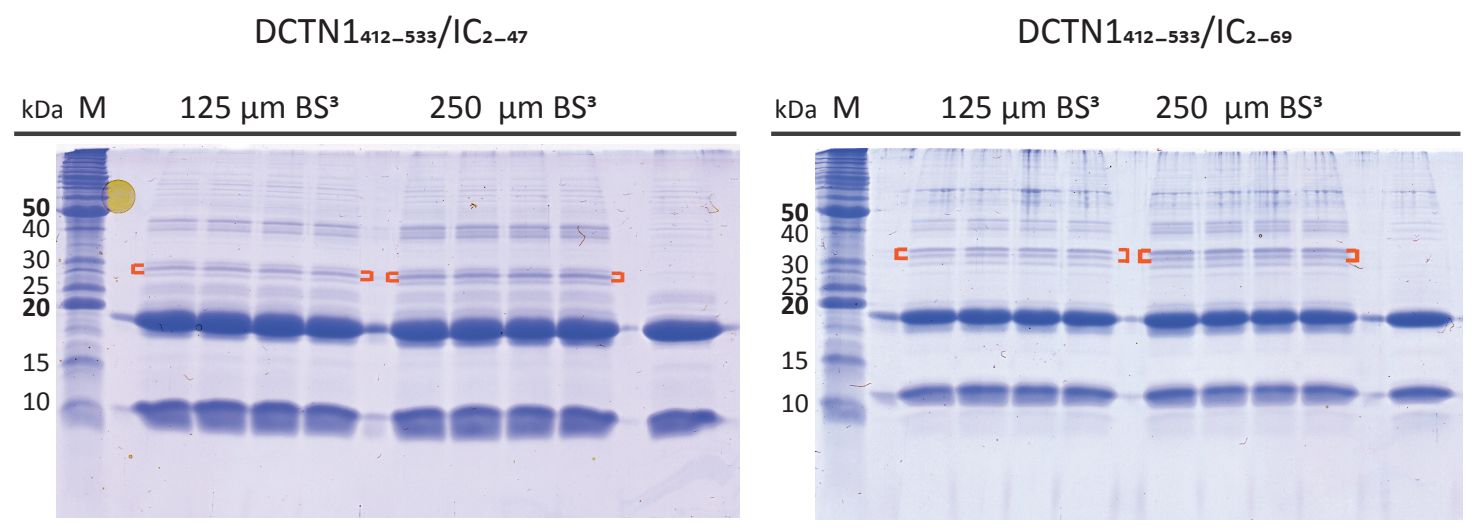

Figure 3.26.: $\mathrm{BS}^{3}$ cross-linked $\mathrm{DCTN1} 1_{412-533}-\mathrm{IC}_{2-47}$ and $\mathrm{IC}_{2-69}$. Shown are $17.5 \%$ SDS gels with cross-linked DCTN1 $1_{412-533}-\mathrm{IC}_{2-47}$ and $\mathrm{DCTN1} 1_{412-533^{-}}$ $\mathrm{IC}_{2-69}$ as well as the non-cross-linked protein complex on the far right of each gel. The orange brackets indicate the bands that were cut from the gel.

Figure 3.27 shows the result of the performed cross-link experiments. Every link that is shown in a black dashed line was found at least once in at least two samples. The exceptions are indicated by a number sign, a asterisk, and a plus and will be explained further down. Example fragmentation spectra for each link can be found in appendix C.2.

The top image (figure 3.27 A) depicts intramolecular links found in dynein IC. BS $^{3}$ formed links between lysines 7 and 13, 27 and 33, and 27 and 34. While the first two links were identified three and four times, respectively, the last one was found 21 times in all experiments. Assuming a canonical $\alpha$-helical structure for the N-terminus with 3.6 residues per turn and $5.4 \AA$ between two turns one can estimate a helical rise of $1.5 \AA$ between two residues. Based on these assumptions none of the cross-linked lysines are more than $10.5 \AA$ apart. Hence, the cross-links are well within the range of the $24 \AA$ (Lys-BS ${ }^{3}$-Lys) that can maximally be bridged (Yang et al., 2012). Since the excised protein band should only contain a DCTN1-IC heterodimer the links found here are on one molecule and not between two IC molecules.

The lower image (figure $3.27 \mathrm{~B}$ ) shows the intermolecular links found between IC and DCTN1. A total of nine links were discovered, many of which were found in more than two out of the six conducted experiments. However, the link marked with \# was 


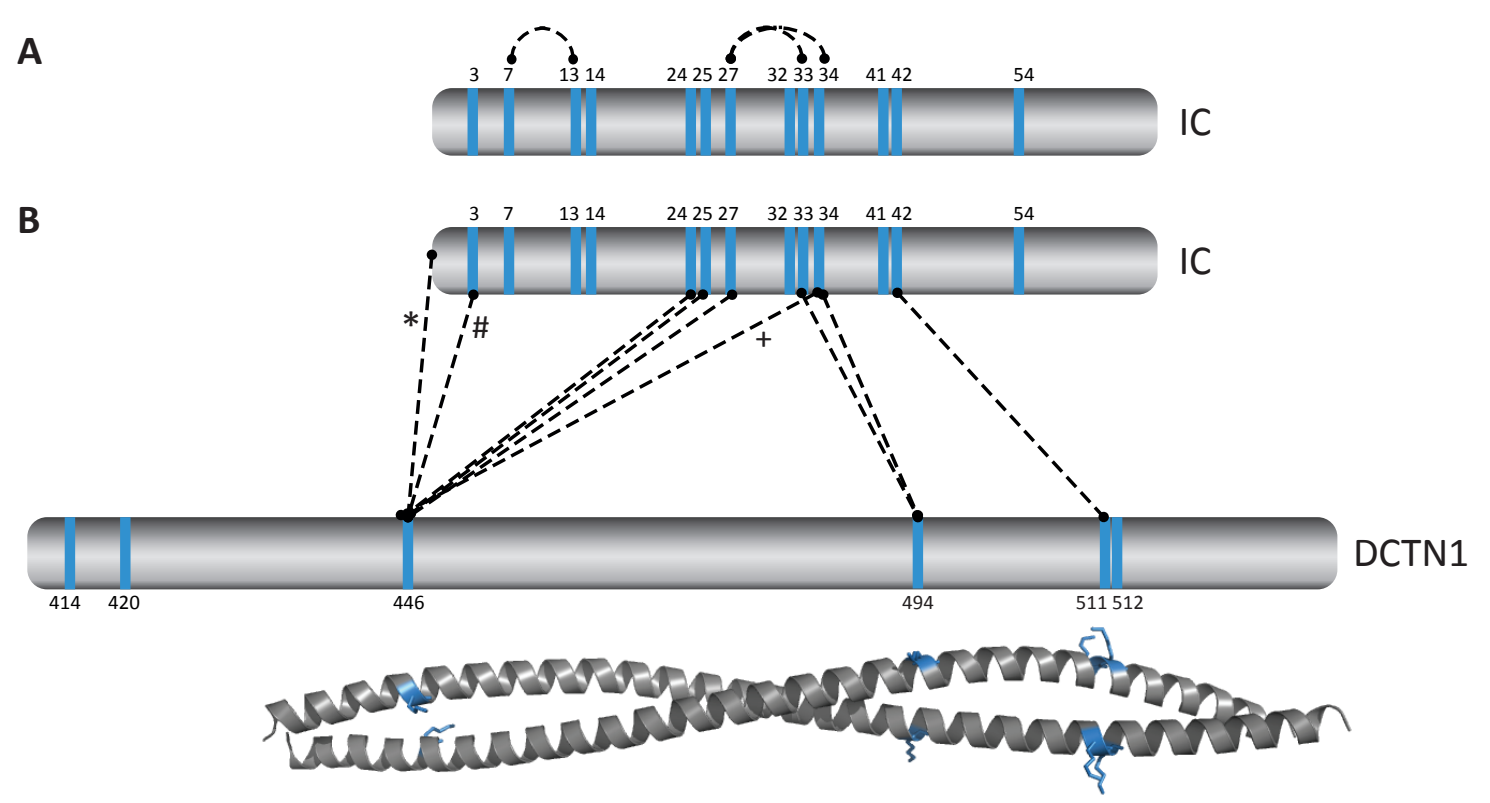

Figure 3.27.: Graphical overview of the cross-link results. (A) Intramolecular cross-links found in IC. (B) Intermolecular cross-links found between IC and DCTN1. The link marked with a "*" was only found twice in one $\mathrm{DCTN1} 1_{412-533}-\mathrm{IC}_{2-69}$ sample. The "\#" sign indicates a link that was found in DCTN1 $142-533-\mathrm{IC}_{2-47}$ samples alone. The "+" was found in two DCTN1 $1_{412-533}-\mathrm{IC}_{2-69}$ samples only. The black dashed lines connect the lysines (blue line) between which the cross-links were found. The numbers indicate the positions of lysines in the corresponding sequence.

only found in $\mathrm{DCTN1} 1_{412-533}-\mathrm{IC}_{2-47}$ samples, whereas * indicates a link identified only twice in one DCTN1 $1_{412-533}-\mathrm{IC}_{2-69}$ sample and ${ }^{+}$is a cross-link found in only two separate DCTN1 $1412-533-\mathrm{IC}_{2-69}$ samples. The first two links found between both molecules are ICN and DCTN1-Lys446, and IC-Lys3 and DCTN1-Lys446. Each link was only found in DCTN1 $1_{412-533}-\mathrm{IC}_{2-69}$ and $\mathrm{DCTN1} 1_{412-533}-\mathrm{IC}_{2-47}$ samples, respectively. The lysine triplet Lys24/25/27 of dynein IC all cross-linked to DCTN1-Lys446 with six, three and nine spectra, respectively, to support each link. The next IC lysine triplet (Lys32/33/34) exhibited cross-links to DCTN1-Lys446 as well as DCTN1-Lys494. In detail, Lys34 only linked to Lys446 of DCTN1. However, Lys33 showed cross-links to both (Lys446 and Lys494). Even though the Lys33-Lys446 link is only supported by two fragmentation spectra found in two DCTN1 ${ }_{412-533}-\mathrm{IC}_{2-69}$ samples, Lys33-Lys494 and Lys34-Lys494 are backed up by more than five spectra each found in different experiments. Due to the distance between Lys446 and Lys494 these cross-links could not have co-existed at the same time but this will be explained later in the discussion chapter. The last link was found between IC-Lys42 and DCTN1-Lys511 with more than 15 fragmentation spectra to support it. 



\section{Discussion}

\subsection{Constructs $\mathrm{DCTN1} 1_{412-533}-\mathrm{IC}_{2-47}$ and DCTN1 $1_{412-533}-\mathrm{IC}_{2-69}$ produced crystals}

Four constructs have been designed to address the question of the dynein-dynactin interaction. These constructs are essentially based on two studies (Morgan et al., 2011, Siglin et al., 2013) that describe new features about the interaction site. The first construct, DCTN1 $1_{412-533}-\mathrm{IC}_{2-47}$, based on gel shift experiments with rat IC and DCTN1 of differing lengths (Siglin et al., 2013), produced house-like crystals that showed diffraction up to $2.9 \AA$. However, a certain amount of IC always got lost during protein purification. To counteract the loss the His-tag formerly on the N-terminus of DCTN1 ${ }_{412-533}$ was transferred to the $\mathrm{N}$-terminus of $\mathrm{IC}_{2-47}$. Subsequent purification steps followed by inspection on SDS gels now showed equal amounts of both proteins. Because $\mathrm{IC}_{2-47}$ is so small that the $8 \times$ His-tag might unfavourably interfere with crystallization a TEV-site was implemented for subsequent removal. Interestingly, crystals appeared after four months only in set-ups with the His-Tag still attached to IC. However, the resolution was too low $(20 \AA)$ to work with the dataset. No crystals developed with His-tagfree protein. Published examples of protein structures where a His-tag was essential for crystallization are rare. In the case of Tajika et al. (2004) the C-terminal His-tag protruded outside the molecule and packed with another molecule, which the authors assumed might have been important for crystallization. Crystals with native (His-tag free) protein could not be produced. However, in more than $90 \%$ of His-tagged solved models the tag was not visible in the structure (Carson et al., 2007) so an influence on the structure or the crystallization can not be ascertained. Moreover, His-tags do not seem to have an effect on the structure as such. As a study that compared native structures and their His-tagged counterparts showed $R$ values and resolution are not effected and only B-values are slightly higher for the tagged structures (Carson et al. 2007). Why no crystals with native $\mathrm{DCTN1} 1_{412-533}-\mathrm{IC}_{2-47}$ appeared cannot be said for sure. A reason could be the missing His-tag since DCTN1 ${ }_{412-533}-\mathrm{IC}_{2-47}$-His-TEV did form crystals albeit with low resolution. Another reason could be that the crystallization condition for the native protein has not been found yet. 
A later study by Morgan et al. (2011) found two IC binding regions. Region 1 includes aa 1-41 of Drosophila melanogaster IC and is assumed to adopt an $\alpha$-helix. Region 2 consists of aa 46-75 and is assumed to form a nascent helix with aa 48-60. Between both regions D. melanogaster IC has several glycines (Gly41-Gly45) that disrupt helices due to high conformational flexibility (Serrano et al., 1992). The transfer of this finding led to the new DCTN1 $1_{412-533}-\mathrm{IC}_{2-84}$ construct. However, after purification the protein complex quickly precipitated. Hence, crystallization set ups were only possible within the next 16 hours after concentration. No crystals formed with this construct. The fast precipitation of $\mathrm{DCTN1}_{412-533}-\mathrm{IC}_{2-84}$ led to the consideration that the extension from 47 aa to 84 aa might have been too long. A comparison between the IC protein sequence of $D$. melanogaster as used in the study and the human IC (see figure 3.5) revealed that the latter has far more prolines in region 2. Human IC contains five prolines in the designated binding region 2 and D. melanogaster only one. Prolines are known to disrupt the helical structure and cause kinks and breaks (Yun et al., 1991). Due to the circular composition of proline it does not have a backbone-NH to take part in a hydrogen bond causing a destabilization of the helix. The consequential kink between two helices varies between 15 to 40\% (Yun et al., 1991). This produces a local flexibility in the helix, which can play an important role in the function of the protein (Visiers et al., 2000). The analysis of human IC shows a proline (Pro45) between the two potential binding regions which might lead to some degree of kinking between the regions. In contrast to D. melanogaster IC region 2 of human IC could only take part in the DCTN1 binding until aa 69 because half of the following ten residues are prolines unlikely to take part in any binding process. Furthermore, these prolines are followed by a disordered protein sequence stretch containing several glycines and prolines.

On these grounds, DCTN1 $142-533-\mathrm{IC}_{2-69}$ was cloned that stopped right before the first proline (Pro70) in region 2. The IC band on SDS gel after gelfiltration was more pronounced compared to $\mathrm{DCTN1} 1_{412-533}-\mathrm{IC}_{2-47}$ indicating a better binding to DCTN1. Crystallization resulted in the same house-like form seen in $\mathrm{DCTN} 1_{412-533}-\mathrm{IC}_{2-47}$ and showed no better resolution (6 A). 


\subsection{Crystallization condition could be responsible for missing IC in crystals}

The missing of bound IC on DCTN1 raised the question what could have caused the separation of both proteins. Protein-protein interactions are established by hydrogen bonds, electrostatics, or hydrophobic bonds. Sedimentation equilibrium experiments carried out by Siglin et al. (2013) showed pH and salt dependence of the DCTN1-IC complex. The complex $\left(\mathrm{DCTN1}_{278-548}\right.$ and $\left.\mathrm{IC}_{1-124}\right)$ was most stable in $100 \mathrm{mM}$ sodium chloride, which is the same salt concentration used for dialysis and gelfiltration buffer in this work. So, the sodium chloride concentration does not impair the DCTN1-IC interaction. Furthermore, the complex showed the highest stability at $\mathrm{pH} 8.0$ with a dissociation constant of $\leq 10^{-14} \mathrm{M}^{2}$ and decreases towards $\mathrm{pH} 7$ and $9\left(\leq 10^{-12} \mathrm{M}^{2}\right.$ and $\leq 10^{-12} \mathrm{M}^{2}$, respectively). Additionally, it should be noted that dissociation constants are also influenced by conditions like temperature and salt. Assuming that at $\mathrm{pH} 5.5$ ( $\mathrm{pH}$ of crystallization condition) the DCTN1-IC interaction will be even weaker and ammonium sulphate (also in crystallization condition) might also have some influence could explain the missing IC molecules in the crystal.

Apart from that, the weaker IC bands visible in SDS gels could indicate that there are two molecular complexes in the sample. One contains both DCTN1 and IC and the other consists only of DCTN1. The principle of gelfiltration is based on the hydrodynamic radius (Stokes radius 1 ) rather than the molecular weight (Erickson, 2009). Because of its elongated shape the hydrodynamic radius of DCTN1 $1_{412-533}$ is large and the addition of IC might not influence the hydrodynamic radius at all or not much. Because of that, in gelfiltration both complexes are eluted at the same time and it would not be possible to distinguish them.

\subsection{DCTN1 $1_{412-533}$ forms a coiled-coil}

The solved structure adopts the expected parallel dimeric left-handed coiled-coil containing 14 continuous heptad motif repeats. Due to 15 SeMet positions and the succession of three alanines in the sequence (Ala485-487), which are well defined in the electron density map of all five helices, the structure could be modelled unambiguously into the electron density.

On average one turn in the $\alpha$-helix is made up of 3.62 residues, which is close to the typical 3.63 residues per turn (Lupas, 1996). The rise per residue (1.51 $\AA$ ) is also in

\footnotetext{
${ }^{1}$ Stokes radius is the radius of a hypothetical hard sphere that has the same diffusion properties like a protein described with the same hydrodynamic radius.
} 
accordance with an average coiled-coil (Phillips, 1992). The helices of the three coiledcoils wind around each other with an average pitch of 149.9 to $158.5 \AA$. Therefore, they belong to the far end of the standard pitch range of 120-160 $\AA$ (Phillips, 1992). Two stretches, one in the $\mathrm{N}$ - and one in the C-terminus, display mean radii. These areas are characterized by mainly canonical hydrophobic residues. Of more interest are two areas found in coiled-coils A/B and C/D that are marked by a slight widening $(>11 \AA)$ followed by a narrowing $(<9 \AA)$ of the distances between the two helices. The first region is characterized by polar amino acids $461 \mathrm{Asn}, 468 \mathrm{Gln}$, and $475 \mathrm{Glu}$ in position $a$ and Asn464 in $d$-position. Although buried polar residues at core positions are thermodynamically not favoured they are important for coiled-coil specificity (Hartmann et al. 2009). Polar residues are known to determine the oligomerization of the leucine zipper GCN4. For example, asparagine in position $a$ is important for the formation of GCN4 parallel dimers (Akey et al. 2001). Furthermore, the substitution of alanine to glutamine or asparagine at position $a$ in tropomyosin could show that the core is less tightly packed (Singh and Hitchcock-DeGregori, 2003) resulting in a larger radius. The second region is characterized by a decrease of the radius. This results from a cluster of three alanines (Ala485, 492, 499) at position $d$. Clusters of alanine have been found before in tropomyosin (Brown et al., 2001) and cortexillin (Burkhard et al., 2000). They are associated with the aforementioned decrease of radius and a stagger between the helices causing a bend of the coiled-coil (Brown et al., 2001). Figure 4.1 shows the alanine cluster superimposed onto a canonical leucine cluster (Leu443, 450, 457) to demonstrate the stagger as seen in Brown et al. (2001). The alanine containing helices are closer together and are slightly out of register, which breaks the 2-fold symmetry seen in canonical (leucine containing) sections of DCTN1 $1412-533$ (depicted in green in figure 4.1. The alanine triplet is bracketed by two local bends at residues Leu482 and Ala504/Asp505. Due to the local opposing direction of the bends (closer to $180^{\circ}$ apart than $0^{\circ}$ ) an in-plane bending can be assigned. While the bending of tropomyosin can be attributed to its need to bind around actin filaments (Brown et al., 2005) it is not clear why dynactin DCTN1 contains such a feature. The E/E' coiled-coil is somewhat different in that it displays a larger $(>6 \AA)$ and longer deviation from the mean radius. This results in a shorter decrease of the radius that does not involve the whole alanine cluster. Also, no local bends could be found with CCBENDS.

The overall structure of the coiled-coils is very similar as indicated by the low r.m.s.d's of the whole chain superimpositions. However, superimposing only the first 29-33 amino acids of the N-terminus resulted in diverging spatial orientations of the coiledcoils which can be attributed to the local bends found in $\mathrm{A} / \mathrm{B}$ and $\mathrm{C} / \mathrm{D}$ but not in 


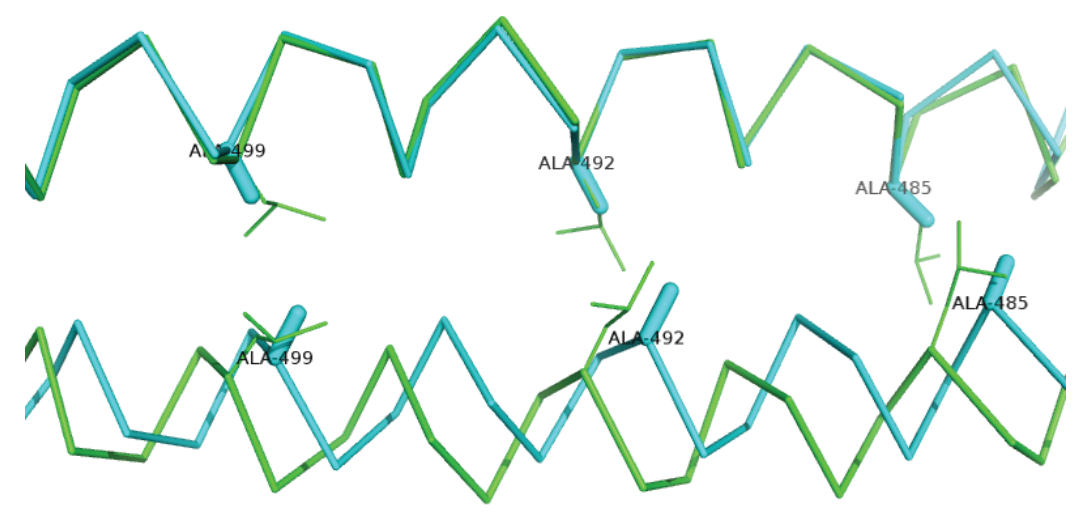

Figure 4.1.: Cluster of three alanines cause stagger in coiled-coil. A canonical leucine section was superimposed with the alanine cluster. It shows that the fragment with alanine in $d$-positions is axially out of register and closer to the other helix. Leucine (green lines) and alanine (blue sticks) in position $d$ are depicted.

E/E'. Furthermore, the rather high B-values are indicative for a highly flexible protein. However, at the sites of crystal contact between coiled-coils the B-values are considerably smaller (see figure 3.20). Studies have questioned whether B-values are actually a predicate for protein dynamics ( tacts are involved. For example, myoglobin crystallized in two different crystal forms resulting in an overall identical structure. But comparison of the B-factors showed significant differences in parts of the structure which resulted from the different packing constraints (Phillips, 1990). The B-factor disturbance coming from crystal contacts are considered as one of the largest noise source in B-factors data (Radivojac et al., 2004), which is why conclusions based on these values could be misleading.

\subsection{N-terminal IC is more likely to be a single $\alpha$-helix than a coiled-coil}

The first 44-67 residues of the N-terminal region of dynein intermediate chains are predicted to form a coiled-coil. However, analyses of the coiled-coil propensity indicate that two single $\alpha$-helices are more likely. The $a$ - and $d$-positions, which are mainly apolar (75-80\%) in coiled-coils (Conway and Parry, 1990), are occupied by only 35\% apolar residues in $\mathrm{IC}_{1-69}$. Instead these positions as well as other heptad positions are filled with charged residues. A total of $56 \%$ of the residues are charged, which is in agreement with 57\% found in the CSAH of myosin 10 (Peckham and Knight, 2009). In contrast DCTN1 $1_{412-533}$ only contains $41 \%$ charged residues consistent with findings in 
the myosin 2 coiled-coil (Peckham and Knight, 2009). These findings favour a CSAH for IC over a coiled-coil. This assumption can be further fortified by a recent publication by Siglin et al. (2013). Molecular weight determined by sedimentation equilibrium analytical ultracentrifugation suggests a monomeric $\mathrm{IC}$ (both for $\mathrm{IC}_{1-44}$ and $\mathrm{IC}_{1-124}$ ).

\subsection{Possible dynein-dynactin interaction models}

The crosslink experiments were performed to clarify the binding location of dynein IC on DCTN1. Based on the present results no definite conclusion can be drawn. However, four interaction scenarios can be imagined, which are depicted in figure 4.2. Two crosslink hotspots could be detected on DCTN1 $1_{412-533}$, one on the N-terminus at Lys446 (green) that is located in the negatively charged area (for surface figure see 3.23) and one on the C-terminus at Lys494 (orange) and Lys511(purple) being in a less negatively charged region. The distance between Lys446 and Lys494 amounts to $\approx 71 \AA$ which cannot be covered by two crosslinks co-existing at the same time on the same IC molecule since one crosslink (Lys-BS ${ }^{3}$-Lys) can maximally bridge $24 \AA$ (Young et al., 2000). Therefore, two binding sites on DCTN1 $1_{412-533}$ are considered. The question is, how dynein IC binds to these sites. Considering that both the short $\mathrm{IC}_{2-47}$, which only comprisef the first binding region (Morgan et al., 2011), and the longer $\mathrm{IC}_{2-69}$ developed crosslinks at the $\mathrm{N}$ - and the $\mathrm{C}$-terminus one can assume that the two IC molecules bind separately to two different DCTN1 $1_{412-533}$ binding sites. For this argument it is of help to assume as discussed above that the N-terminal IC region will not adopt a coiled-coil structure but rather bind individually to DCTN1. Because the first 60 residues of the N-terminal IC adopt an $\alpha$-helix or a nascent helix (Morgan et al. 2011) the length of IC in the image is scaled to the length of a perfect $\alpha$-helix (1.5 $\AA$ rise per residue (Lupas and Gruber, 2005)). The IC molecules have been placed in such a way that the first and the last possible link (according to each colour) are each no more than $24 \AA$ from the DCTN1 lysine apart.

There are two possible IC binding positions on both DCTN1 $1_{412-533}$ sites. One is a parallel binding of the IC molecules related to the direction of the DCTN1 $1_{412-533}$ coiled-coil (like in figure $4.2 \mathrm{~A}$ ). In the case of the N-terminal bound IC molecule the distance to the first and last (Lys3 and Lys35) crosslink of IC each is $24 \AA$. Hence, there is no other possible location when taking all N-terminal crosslinks into account. The situation is different for the C-terminal parallel bound molecule. Here, the drawn-in 


\section{A Parallel binding}

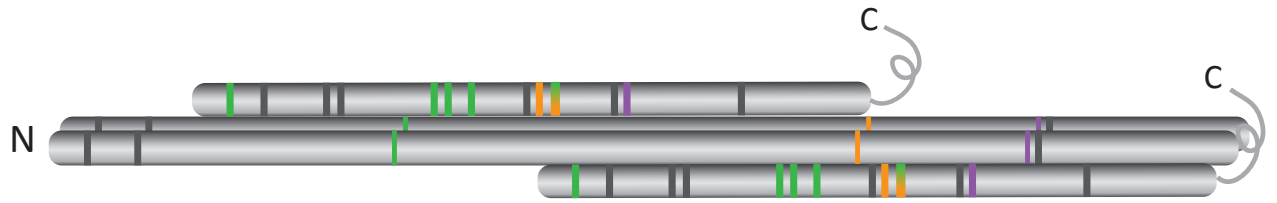

B Antiparallel binding

C

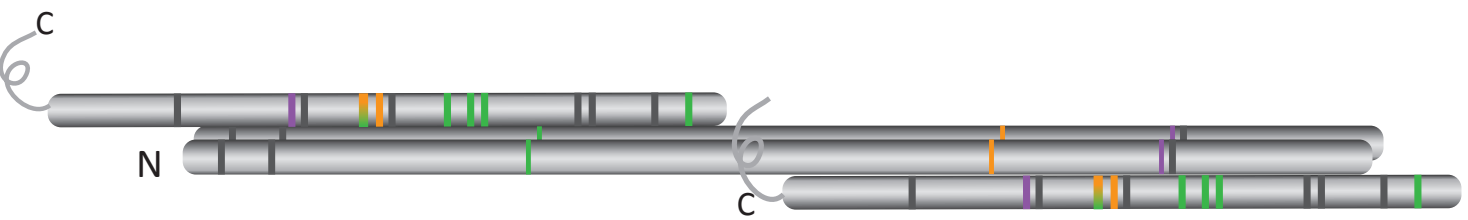

C

\section{Parallel/anti-parallel binding}

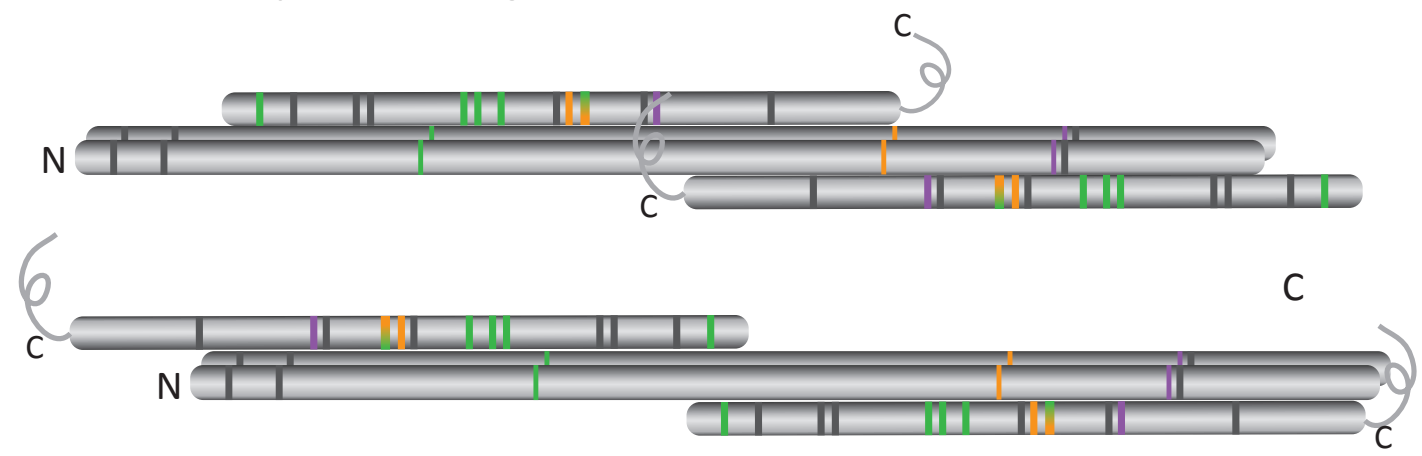

\section{Legend}

IC

$\begin{array}{lllllll}3 & 7 & 1314 & 242527 & 323334 & 4142 & 54\end{array}$

DCTN

414420

446

511512

Figure 4.2.: Possible dynein-dynactin interaction models. The image shows three possible scenarios for the binding of dynein IC on DCTN1. Each bar represents a lysine position. Same coloured lysines in IC crosslinked to the DCTN1 $1_{412-533}$ lysine in the same color. Bars in grey did not exhibit any crosslinks. For reasons of simplicity only $\mathrm{IC}_{2-69}$ and not $\mathrm{IC}_{2-47}$ is shown. Both proteins are drawn in the scale of a perfect $\alpha$-helix. (A) Both, the DCTN1 $1_{412-533}$ coiled-coil and the single IC monomers bind parallel (in the same $\mathrm{N} \rightarrow \mathrm{C}$ direction). (B) Both IC molecules bind anti-parallel to $\mathrm{DCTN}_{412-533}$. (C) One IC molecule binds parallel, the other anti-parallel to $\mathrm{DCTN}_{412-533}$.

molecule could be shifted towards both termini (within the $24 \AA$ constraints). The other possibility is anti-parallel bound IC molecules (like in figure $4.2 \mathrm{~B}$ ). Again,there is not much room for placing the N-terminally bound IC molecule to DCTN1 $1_{412-533}$ because of the crosslink restraints. Of note here is the absence of some crosslinks (dark 
grey). Although not all expected crosslinks can form due to geometrical restraints it is remarkable that -if anti-parallel binding does exist- not even one crosslink between the three apparent proximal IC lysines (Lys41, 42, 54) and the surface Lys414 and 420 of DCTN1 $1_{412-533}$ was found. However, the same consideration can also be applied to the parallel C-terminal bound IC molecule (e.g. no link of Lys24-27 to DCTN1 $1_{412-533}$ Lys494) as well as the anti-parallel C-terminal bound IC molecule (e.g. no link of Lys24-27 to DCTN1 $1_{412-533}$ Lys511). Furthermore, due to the cross-over crosslinks in the anti-parallel C-terminal bound IC molecule (IC Lys52 to DCTN1 Lys494 and IC Lys33/34 to DCTN1 Lys511) the location of the molecule could not be shifted towards both termini as much as the parallel bound IC.

The first scenario (depicted in figure $4.2 \mathrm{~A}$ ) shows the parallel binding of both $\mathrm{IC}_{2-69}$ molecules onto DCTN1 $1_{412-533}$. The second possibility (figure $4.2 \mathrm{~B}$ ) consists of the antiparallel binding of both IC molecules to the $\mathrm{DCTN1}_{412-533}$ coiled-coil. The last scenario (figure $4.2 \mathrm{C}$ ) depicts a parallel/anti-parallel binding. It is twofold as the IC molecule on the N-terminus can bind both ways and the molecule on the C-terminus in each case binds the opposite way. In order to estimate which interaction scenario is most suitable the (minimal and maximal) distance between the two C-termini of the IC molecules can be compared. For the function of the whole IC molecule it is vital that the two molecules are not too far apart since they both bind the dynein light chain Tctex-1 starting at residue Leu124 (Mok et al., 2001). So, after less than 60 residues both molecules have to be in close enough proximity $(\approx 20 \AA$, average distance between the two IC molecules when bound to Tctex-1) to bind to Tctex-1. Applying this criterion to the four possibilities shows that the second parallel/anti-parallel scenario with IC C-terminal distances of $\approx 163-192 \AA$ cannot fulfil it. Although it is not possible to determine the length of the disordered section (aa70-123) it is unlikely that about $190 \AA$ are bridged by a disordered region to come into close proximity for binding Tctex1. The C-termini in the anti-parallel scenario have to traverse $\approx 108-117 \AA$, which is about the length of the bound IC molecule. This is more likely but it still seems a very large distance to cross before binding to the protein. However, the parallel (15-49 $\AA$ ) and the first parallel/anti-parallel (7-15 $\AA$ ) scenario could certainly bridge the distance.

In this dissertation the structure of the dimeric DCTN1 $1_{412-533}$ could be solved. It exhibits a canonical coiled-coil structure which features a mainly negatively charged surface. An electrostatical interaction of these dynactin regions with two separately binding dynein ICs is probable. Due to cross-linking experiments the localization of the interaction site could be further refined compared to previous studies. 


\section{Bibliography}

D. L. Akey, V. N. Malashkevich, and P. S. Kim. Buried polar residues in coiled-coil interfaces. Biochemistry, 40(21):6352-6360, May 2001.

D. J. Asai and M. P. Koonce. The dynein heavy chain: structure, mechanics and evolution. Trends in Cell Biology, 11(5):196-202, May 2001.

E. Barbar. Dynein light chain LC8 is a dimerization hub essential in diverse protein networks. Biochemistry, 47(2):503-508, Jan. 2008.

G. Benison, A. Nyarko, and E. Barbar. Heteronuclear NMR identifies a nascent helix in intrinsically disordered dynein intermediate chain: implications for folding and dimerization. J. Mol. Biol., 362(5):1082-1093, Oct. 2006.

G. Bertani. Studies on lysogenesis I. The mode of phage liberation by lysogenic escherichia coli. J Bacteriol, 62(3):293-300, Sept. 1951.

J. B. Bingham and T. A. Schroer. Self-regulated polymerization of the actin-related protein arp1. Curr Biol, 9(4):223-6, 1999.

A. Blangy, L. Arnaud, and E. A. Nigg. Phosphorylation by p34cdc2 protein kinase regulates binding of the kinesin-related motor HsEg5 to the dynactin subunit p150. J Biol Chem, 272(31):19418-19424, 1997.

M. M. Bradford. A rapid and sensitive method for the quantitation of microgram quantities of protein utilizing the principle of protein-dye binding. Anal. Biochem., 72:248-254, May 1976.

A. T. Brünger, P. D. Adams, G. M. Clore, W. L. DeLano, P. Gros, R. W. GrosseKunstleve, J. S. Jiang, J. Kuszewski, M. Nilges, N. S. Pannu, R. J. Read, L. M. Rice, T. Simonson, and G. L. Warren. Crystallography \& NMR system: A new software suite for macromolecular structure determination. Acta Crystallogr. D Biol. Crystallogr., 54(Pt 5):905-921, Sept. 1998.

J. H. Brown. How sequence directs bending in tropomyosin and other two-stranded alpha-helical coiled coils. Protein Sci., 19(7):1366-1375, July 2010. 
J. H. Brown, K.-H. Kim, G. Jun, N. J. Greenfield, R. Dominguez, N. Volkmann, S. E. Hitchcock-DeGregori, and C. Cohen. Deciphering the design of the tropomyosin molecule. Proc Natl Acad Sci U S A, 98(15):8496-8501, July 2001.

J. H. Brown, Z. Zhou, L. Reshetnikova, H. Robinson, R. D. Yammani, L. S. Tobacman, and C. Cohen. Structure of the mid-region of tropomyosin: Bending and binding sites for actin. Proc Natl Acad Sci U S A, 102(52):18878-18883, Dec. 2005.

X. Bruker. Space group determination and data statistics (XPREP). URL http: //www.bruker.com/products/x-ray-diffraction-and-elemental-analysis/ single-crystal-x-ray-diffraction/sc-xrd-software/ overview/sc-xrd-software/proteum2/proteum2-software/ space-group-determination-and-data-statistics.html.

P. Burkhard, R. A. Kammerer, M. O. Steinmetz, G. P. Bourenkov, and U. Aebi. The coiled-coil trigger site of the rod domain of cortexillin i unveils a distinct network of interhelical and intrahelical salt bridges. Structure, 8(3):223-230, Mar. 2000.

J. K. Burkhardt, C. J. Echeverri, T. Nilsson, and R. B. Vallee. Overexpression of the dynamitin (p50) subunit of the dynactin complex disrupts dynein-dependent maintenance of membrane organelle distribution. The Journal of Cell Biology, 139 (2):469 -484, Oct. 1997.

M. Carson, D. H. Johnson, H. McDonald, C. Brouillette, and L. J. DeLucas. His-tag impact on structure. Acta Crystallographica Section D Biological Crystallography, 63(3):295-301, Mar. 2007.

A. P. Carter. Crystal clear insights into how the dynein motor moves. J. Cell. Sci., 126(Pt 3):705-713, Feb. 2013.

A. P. Carter, C. Cho, L. Jin, and R. D. Vale. Crystal structure of the dynein motor domain. Science, 331(6021):1159-1165, Mar. 2011.

CCP4. The CCP4 suite: programs for protein crystallography. Acta Crystallogr. D Biol. Crystallogr., 50(Pt 5):760-763, Sept. 1994.

V. B. Chen, W. B. Arendall, 3rd, J. J. Headd, D. A. Keedy, R. M. Immormino, G. J. Kapral, L. W. Murray, J. S. Richardson, and D. C. Richardson. MolProbity: allatom structure validation for macromolecular crystallography. Acta Crystallogr. D Biol. Crystallogr., 66(Pt 1):12-21, Jan. 2010. 
S. N. Cohen and A. C. Chang. Recircularization and autonomous replication of a sheared r-factor DNA segment in escherichia coli transformants. Proc. Natl. Acad. Sci. U.S.A., 70(5):1293-1297, May 1973.

J. F. Conway and D. A. Parry. Structural features in the heptad substructure and longer range repeats of two-stranded $\alpha$-fibrous proteins. International Journal of Biological Macromolecules, 12(5):328-334, Oct. 1990.

G. E. Crooks, G. Hon, J. M. Chandonia, and S. E. Brenner. WebLogo: a sequence logo generator. Genome Res, 14:1188 - 1190, 2004.

T. L. Culver-Hanlon, S. A. Lex, A. D. Stephens, N. J. Quintyne, and S. J. King. A microtubule-binding domain in dynactin increases dynein processivity by skating along microtubules. Nat Cell Biol, 8(3):264-70, 2006.

S. W. Deacon, A. S. Serpinskaya, P. S. Vaughan, M. L. Fanarraga, I. Vernos, K. T. Vaughan, and V. I. Gelfand. Dynactin is required for bidirectional organelle transport. J Cell Biol, 160(3):297-301, Feb. 2003.

M. Delorenzi and T. Speed. An HMM model for coiled-coil domains and a comparison with PSSM-based predictions. Bioinformatics, 18(4):617-625, Apr. 2002.

F. DiMaio, D. A. Kondrashov, E. Bitto, A. Soni, C. A. Bingman, G. N. Phillips, Jr, and J. W. Shavlik. Creating protein models from electron-density maps using particle-filtering methods. Bioinformatics, 23(21):2851-2858, Nov. 2007.

C. J. Echeverri, B. M. Paschal, K. T. Vaughan, and R. B. Vallee. Molecular characterization of the $50-\mathrm{kD}$ subunit of dynactin reveals function for the complex in chromosome alignment and spindle organization during mitosis. J Cell Biol, 132(4): 617-33, 1996.

C. Eckert, A. Goretzki, M. Faberova, and M. Kollmar. Conservation and divergence between cytoplasmic and muscle-specific actin capping proteins: insights from the crystal structure of cytoplasmic cap32/34 from dictyostelium discoideum. BMC Struct. Biol., 12(1):12, June 2012.

D. M. Eckley, S. R. Gill, K. A. Melkonian, J. B. Bingham, H. V. Goodson, J. E. Heuser, and T. A. Schroer. Analysis of dynactin subcomplexes reveals a novel actinrelated protein associated with the arp1 minifilament pointed end. J Cell Biol, 147 (2):307-320, 1999.

P. Emsley and K. Cowtan. Coot : model-building tools for molecular graphics. Acta Crystallographica Section D Biological Crystallography, 60(12):2126-2132, Nov. 2004. 
P. Emsley, B. Lohkamp, W. G. Scott, and K. Cowtan. Features and development of coot. Acta Crystallogr. D Biol. Crystallogr, 66(Pt 4):486-501, Apr. 2010.

H. P. Erickson. Size and shape of protein molecules at the nanometer level determined by sedimentation, gel filtration, and electron microscopy. Biol Proced Online, 11: 32-51, May 2009.

G. Fairbanks, T. L. Steck, and D. F. Wallach. Electrophoretic analysis of the major polypeptides of the human erythrocyte membrane. Biochemistry, 10(13):2606-2617, June 1971.

E. A. Foley and T. M. Kapoor. Microtubule attachment and spindle assembly checkpoint signalling at the kinetochore. Nat. Rev. Mol. Cell Biol., 14(1):25-37, Jan. 2013.

J. A. Garces, I. B. Clark, D. I. Meyer, and R. B. Vallee. Interaction of the p62 subunit of dynactin with arp1 and the cortical actin cytoskeleton. Curr Biol, 9(24):1497-1500, 1999.

I. R. Gibbons and A. J. Rowe. Dynein: A protein with adenosine triphosphatase activity from cilia. Science, 149(3682):424-426, July 1965.

S. R. Gill, T. A. Schroer, I. Szilak, E. R. Steuer, M. P. Sheetz, and D. W. Cleveland. Dynactin, a conserved, ubiquitously expressed component of an activator of vesicle motility mediated by cytoplasmic dynein. J Cell Biol, 115(6):1639-1650, Dec. 1991.

J. Hall, P. A. Karplus, and E. Barbar. Multivalency in the assembly of intrinsically disordered dynein intermediate chain. J. Biol. Chem., 284(48):33115-33121, Nov. 2009 .

J. Hall, Y. Song, P. A. Karplus, and E. Barbar. The crystal structure of dynein intermediate chain-light chain roadblock complex gives new insights into dynein assembly. J. Biol. Chem., 285(29):22566-22575, July 2010.

T. A. Hall. BioEdit: a user-friendly biological sequence alignment editor and analysis program for windows 95/98/NT. Nucleic Acids Symp. Ser., 41:95-98, 1999.

B. Halle. Flexibility and packing in proteins. Proc. Natl. Acad. Sci. U.S.A., 99(3): 1274-1279, Feb. 2002.

B. Hammesfahr and M. Kollmar. Evolution of the eukaryotic dynactin complex, the activator of cytoplasmic dynein. BMC Evol. Biol., 12(1):95, June 2012. 
A. Harada, Y. Takei, Y. Kanai, Y. Tanaka, S. Nonaka, and N. Hirokawa. Golgi vesiculation and lysosome dispersion in cells lacking cytoplasmic dynein. J Cell Biol, 141 (1):51-9, 1998 .

M. A. Hartman and J. A. Spudich. The myosin superfamily at a glance. J. Cell. Sci., 125(Pt 7):1627-1632, Apr. 2012.

M. D. Hartmann, O. Ridderbusch, K. Zeth, R. Albrecht, O. Testa, D. N. Woolfson, G. Sauer, S. Dunin-Horkawicz, A. N. Lupas, and B. H. Alvarez. A coiled-coil motif that sequesters ions to the hydrophobic core. Proc. Natl. Acad. Sci. U.S.A., 106(40): 16950-16955, Oct. 2009.

K. Hatje, B. Hammesfahr, and M. Kollmar. WebScipio: reconstructing alternative splice variants of eukaryotic proteins. Nucleic Acids Res., 41(Web Server issue): W504-509, July 2013.

A. G. Hendricks, J. E. Lazarus, E. Perlson, M. K. Gardner, D. J. Odde, Y. E. Goldman, and E. L. F. Holzbaur. Dynein tethers and stabilizes dynamic microtubule plus ends. Curr. Biol., 22(7):632-637, Apr. 2012.

W. A. Hendrickson and C. M. Ogata. [28] Phase determination from multiwavelength anomalous diffraction measurements. In C. W. Carter, editor, Methods in Enzymology, volume 276 of Macromolecular Crystallography Part A, pages 494-523. Academic Press, 1997. ISBN 0076-6879.

W. A. Hendrickson, J. R. Horton, and D. M. LeMaster. Selenomethionyl proteins produced for analysis by multiwavelength anomalous diffraction (MAD): a vehicle for direct determination of three-dimensional structure. EMBO J., 9(5):1665-1672, May 1990.

N. Hirokawa, Y. Noda, Y. Tanaka, and S. Niwa. Kinesin superfamily motor proteins and intracellular transport. Nat Rev Mol Cell Biol, 10(10):682-696, Oct. 2009.

E. A. Holleran, L. A. Ligon, M. Tokito, M. C. Stankewich, J. S. Morrow, and E. L. Holzbaur. beta III spectrin binds to the arp1 subunit of dynactin. J Biol Chem, 276 (39):36598-605, 2001.

E. L. F. Holzbaur, J. A. Hammarback, B. M. Paschal, N. G. Kravit, K. K. Pfister, and R. B. Vallee. Homology of a $150 \mathrm{~K}$ cytoplasmic dynein-associated polypeptide with the drosophila gene glued. Nature, 351(6327):579-583, June 1991. 
S. Honnappa, O. Okhrimenko, R. Jaussi, H. Jawhari, I. Jelesarov, F. K. Winkler, and M. O. Steinmetz. Key interaction modes of dynamic +TIP networks. Mol Cell, 23 (5):663-671, 2006.

B. J. Howell, B. F. McEwen, J. C. Canman, D. B. Hoffman, E. M. Farrar, C. L. Rieder, and E. D. Salmon. Cytoplasmic dynein/dynactin drives kinetochore protein transport to the spindle poles and has a role in mitotic spindle checkpoint inactivation. $J$ Cell Biol, 155(7):1159-1172, 2001.

W. Kabsch. XDS. Acta Crystallogr. D Biol. Crystallogr, 66(Pt 2):125-132, Feb. 2010.

K. A. Kantardjieff and B. Rupp. Matthews coefficient probabilities: Improved estimates for unit cell contents of proteins, DNA, and protein-nucleic acid complex crystals. Protein Sci., 12(9):1865-1871, Sept. 2003.

J. R. Kardon and R. D. Vale. Regulators of the cytoplasmic dynein motor. Nat. Rev. Mol. Cell Biol, 10(12):854-865, Dec. 2009.

S. Karki and E. L. Holzbaur. Cytoplasmic dynein and dynactin in cell division and intracellular transport. Curr Opin Cell Biol, 11(1):45-53, 1999.

S. Karki, B. LaMonte, and E. L. Holzbaur. Characterization of the p22 subunit of dynactin reveals the localization of cytoplasmic dynein and dynactin to the midbody of dividing cells. J Cell Biol, 142(4):1023-34, 1998.

S. Karki, M. K. Tokito, and E. L. Holzbaur. A dynactin subunit with a highly conserved cysteine-rich motif interacts directly with arp1. J Biol Chem, 275(7):4834-9, 2000.

S. J. King, C. L. Brown, K. C. Maier, N. J. Quintyne, and T. A. Schroer. Analysis of the dynein-dynactin interaction in vitro and in vivo. Mol. Biol. Cell, 14(12): 5089-5097, Dec. 2003.

T. Kon, M. Nishiura, R. Ohkura, Y. Y. Toyoshima, and K. Sutoh. Distinct functions of nucleotide-binding/hydrolysis sites in the four AAA modules of cytoplasmic dynein. Biochemistry, 43(35):11266-11274, Sept. 2004.

T. Kon, K. Sutoh, and G. Kurisu. X-ray structure of a functional full-length dynein motor domain. Nat. Struct. Mol. Biol, 18(6):638-642, June 2011.

F. J. Kull, R. D. Vale, and R. J. Fletterick. The case for a common ancestor: kinesin and myosin motor proteins and g proteins. J. Muscle Res. Cell. Motil., 19(8):877886, Nov. 1998. 
A. Kuta, W. Deng, A. Morsi El-Kadi, G. T. Banks, M. Hafezparast, K. K. Pfister, and E. M. C. Fisher. Mouse cytoplasmic dynein intermediate chains: Identification of new isoforms, alternative splicing and tissue distribution of transcripts. PLoS ONE, 5(7):e11682, July 2010.

U. K. Laemmli. Cleavage of structural proteins during the assembly of the head of bacteriophage t4. Nature, 227(5259):680-685, Aug. 1970.

I. H. Lee, S. Kumar, and M. Plamann. Null mutants of the neurospora actin-related protein 1 pointed-end complex show distinct phenotypes. Mol Biol Cell, 12(7):2195206, 2001.

L. A. Ligon, S. S. Shelly, M. Tokito, and E. L. F. Holzbaur. The microtubule plus-end proteins EB1 and dynactin have differential effects on microtubule polymerization. Mol. Biol. Cell, 14(4):1405-1417, Apr. 2003.

C. M. Lim, M. A. Cater, J. F. B. Mercer, and S. La Fontaine. Copper-dependent interaction of dynactin subunit p62 with the $\mathrm{n}$ terminus of ATP7B but not ATP7A. J Biol. Chem., 281(20):14006 -14014, May 2006.

A. Lupas. Coiled coils: new structures and new functions. Trends Biochem. Sci., 21 (10):375-382, Oct. 1996.

A. N. Lupas and M. Gruber. The structure of alpha-helical coiled coils. Adv. Protein Chem., 70:37-78, 2005.

M. Makokha, M. Hare, M. Li, T. Hays, and E. Barbar. Interactions of cytoplasmic dynein light chains tctex-1 and LC8 with the intermediate chain IC74. Biochemistry, 41(13):4302-4311, Apr. 2002.

B. W. Matthews. Solvent content of protein crystals. J. Mol. Biol, 33(2):491-497, Apr. 1968.

A. J. McCoy, R. W. Grosse-Kunstleve, P. D. Adams, M. D. Winn, L. C. Storoni, and R. J. Read. Phaser crystallographic software. Journal of Applied Crystallography, 40(4):658-674, Aug. 2007.

M. McGrail, J. Gepner, A. Silvanovich, S. Ludmann, M. Serr, and T. S. Hays. Regulation of cytoplasmic dynein function in vivo by the drosophila glued complex. J Cell Biol, 131(2):411-25, 1995.

Y.-K. Mok, K. W.-H. Lo, and M. Zhang. Structure of tctex-1 and its interaction with cytoplasmic dynein intermediate chain. J. Biol. Chem., Jan. 2001. 
J. L. Morgan, Y. Song, and E. Barbar. Structural dynamics and multi-region interactions in dynein-dynactin recognition. J Biol. Chem., 2011.

G. N. Murshudov, P. Skubák, A. A. Lebedev, N. S. Pannu, R. A. Steiner, R. A. Nicholls, M. D. Winn, F. Long, and A. A. Vagin. REFMAC5 for the refinement of macromolecular crystal structures. Acta Crystallogr. D Biol. Crystallogr., 67(Pt 4): 355-367, Apr. 2011.

K. Nath and B. A. Azzolina. Cleavage properties of site-specific restriction endonucleases. Gene Amplif Anal, 1:113-130, 1981.

A. F. Neuwald, L. Aravind, J. L. Spouge, and E. V. Koonin. AAA+: a class of chaperone-like ATPases associated with the assembly, operation, and disassembly of protein complexes. Genome Res., 9(1):27-43, Jan. 1999.

F. Odronitz and M. Kollmar. Drawing the tree of eukaryotic life based on the analysis of 2,269 manually annotated myosins from 328 species. Genome Biol, 8(9):R196, 2007.

L. R. Otterbein, P. Graceffa, and R. Dominguez. The crystal structure of uncomplexed actin in the ADP state. Science, 293(5530):708-711, July 2001.

G. Parisi, M. S. Fornasari, and J. Echave. Dynactins p25 and p27 are predicted to adopt the LBH fold. FEBS Letters, 562(1-3):1-4, Mar. 2004.

G. J. Pazour, B. L. Dickert, and G. B. Witman. The DHC1b (DHC2) isoform of cytoplasmic dynein is required for flagellar assembly. J. Cell Biol., 144(3):473-481, Feb. 1999.

M. Peckham and P. J. Knight. When a predicted coiled coil is really a single $\alpha$-helix, in myosins and other proteins. Soft Matter, 2009.

K. K. Pfister, P. R. Shah, H. Hummerich, A. Russ, J. Cotton, A. A. Annuar, S. M. King, and E. M. C. Fisher. Genetic analysis of the cytoplasmic dynein subunit families. PLoS Genet, 2(1):e1, Jan. 2006.

G. N. Phillips. What is the pitch of the $\alpha$-helical coiled coil? Proteins, 14(4):425-429, Dec. 1992.

G. N. Phillips, Jr. Comparison of the dynamics of myoglobin in different crystal forms. Biophys. J., 57(2):381-383, Feb. 1990. 
J. F. Presley, N. B. Cole, T. A. Schroer, K. Hirschberg, K. J. Zaal, and J. LippincottSchwartz. ER-to-Golgi transport visualized in living cells. Nature, 389(6646):81-5, 1997.

P. Radivojac, Z. Obradovic, D. K. Smith, G. Zhu, S. Vucetic, C. J. Brown, J. D. Lawson, and A. K. Dunker. Protein flexibility and intrinsic disorder. Protein Sci., 13(1):71-80, Jan. 2004.

J. Rappsilber, S. Siniossoglou, E. C. Hurt, and M. Mann. A generic strategy to analyze the spatial organization of multi-protein complexes by cross-linking and mass spectrometry. Anal. Chem., 72(2):267-275, Jan. 2000.

A. J. Roberts, T. Kon, P. J. Knight, K. Sutoh, and S. A. Burgess. Functions and mechanics of dynein motor proteins. Nat. Rev. Mol. Cell Biol., 14(11):713-726, Nov. 2013.

R. K. Saiki, D. H. Gelfand, S. Stoffel, S. J. Scharf, R. Higuchi, G. T. Horn, K. B. Mullis, and H. A. Erlich. Primer-directed enzymatic amplification of DNA with a thermostable DNA polymerase. Science, 239(4839):487-491, Jan. 1988.

D. A. Schafer, S. R. Gill, J. A. Cooper, J. E. Heuser, and T. A. Schroer. Ultrastructural analysis of the dynactin complex: an actin-related protein is a component of a filament that resembles f-actin. J Cell Biol, 126(2):403-12, 1994a.

D. A. Schafer, Y. O. Korshunova, T. A. Schroer, and J. A. Cooper. Differential localization and sequence analysis of capping protein beta-subunit isoforms of vertebrates. J Cell Biol, 127(2):453-465, 1994b.

M. Schliwa and G. Woehlke. Molecular motors. Nature, 422(6933):759-65, 2003.

T. D. Schneider and R. M. Stephens. Sequence logos: a new way to display consensus sequences. Nucleic Acids Res., 18(20):6097-6100, Oct. 1990.

T. A. Schroer. Dynactin. Annu Rev Cell Dev Biol, 20:759-779, 2004.

T. A. Schroer and M. P. Sheetz. Two activators of microtubule-based vesicle transport. J Cell Biol, 115(5):1309-1318, 1991.

L. Serrano, J.-L. Neira, J. Sancho, and A. R. Fersht. Effect of alanine versus glycine in $\alpha$-helices on protein stability. Nature, 356(6368):453-455, Apr. 1992.

P. A. Sharp, B. Sugden, and J. Sambrook. Detection of two restriction endonuclease activities in haemophilus parainfluenzae using analytical agarose-ethidium bromide electrophoresis. Biochemistry, 12(16):3055-3063, July 1973. 
G. M. Sheldrick. Experimental phasing with SHELXC / D / E : combining chain tracing with density modification. Acta Crystallographica Section D Biological Crystallography, 66(4):479-485, Mar. 2010.

A. E. Siglin. Biophysical and in vivo characterization of the dynein-dynactin interaction; implications for multiple modes of regulation. $\mathrm{PhD}$ thesis, THOMAS JEFFERSON UNIVERSITY, 2010.

A. E. Siglin, S. Sun, J. K. Moore, S. Tan, M. Poenie, J. D. Lear, T. Polenova, J. A. Cooper, and J. C. Williams. Dynein and dynactin leverage their bivalent character to form a high-affinity interaction. PLoS ONE, 8(4):e59453, 2013.

A. Singh and S. E. Hitchcock-DeGregori. Local destabilization of the tropomyosin coiled coil gives the molecular flexibility required for actin binding. Biochemistry, 42 (48):14114-14121, Dec. 2003.

M. C. Smith, T. C. Furman, T. D. Ingolia, and C. Pidgeon. Chelating peptideimmobilized metal ion affinity chromatography. a new concept in affinity chromatography for recombinant proteins. J. Biol. Chem., 263(15):7211-7215, May 1988.

S. V. Strelkov and P. Burkhard. Analysis of alpha-helical coiled coils with the program TWISTER reveals a structural mechanism for stutter compensation. J. Struct. Biol., 137(1-2):54-64, Feb. 2002.

A. Sugino, H. M. Goodman, H. L. Heyneker, J. Shine, H. W. Boyer, and N. R. Cozzarelli. Interaction of bacteriophage t4 RNA and DNA ligases in joining of duplex DNA at base-paired ends. J. Biol. Chem., 252(11):3987-3994, June 1977.

S. J. Susalka, K. Nikulina, M. W. Salata, P. S. Vaughan, S. M. King, K. T. Vaughan, and K. K. Pfister. The roadblock light chain binds a novel region of the cytoplasmic dynein intermediate chain. J. Biol. Chem., 277(36):32939-32946, Sept. 2002.

D. Süveges, Z. Gáspári, G. Tóth, and L. Nyitray. Charged single $\alpha$-helix: A versatile protein structural motif. Proteins, 74(4):905-916, Mar. 2009.

Y. Tajika, N. Sakai, T. Tamura, M. Yao, N. Watanabe, and I. Tanaka. Crystal structure of hypothetical protein PH0828 from pyrococcus horikoshii. Proteins, 57(4):862-865, Dec. 2004.

M. Terasawa, M. Toya, F. Motegi, M. Mana, K. Nakamura, and A. Sugimoto. Caenorhabditis elegans ortholog of the p24/p22 subunit, DNC-3, is essential for the formation of the dynactin complex by bridging DNC-1/p150Glued and DNC2/dynamitin. Genes to Cells, 15(11):1145-1157, Nov. 2010. 
S. H. Tynan, M. A. Gee, and R. B. Vallee. Distinct but overlapping sites within the cytoplasmic dynein heavy chain for dimerization and for intermediate chain and light intermediate chain binding. J. Biol. Chem., 275(42):32769-32774, Oct. 2000.

E. A. Vaisberg, M. P. Koonce, and J. R. McIntosh. Cytoplasmic dynein plays a role in mammalian mitotic spindle formation. J Cell Biol, 123(4):849-58, 1993.

E. A. Vaisberg, P. M. Grissom, and J. R. McIntosh. Mammalian cells express three distinct dynein heavy chains that are localized to different cytoplasmic organelles. J. Cell Biol., 133(4):831-842, May 1996.

K. T. Vaughan and R. B. Vallee. Cytoplasmic dynein binds dynactin through a direct interaction between the intermediate chains and p150Glued. J. Cell Biol., 131(6 Pt 1):1507-1516, Dec. 1995.

K. T. Vaughan, S. H. Tynan, N. E. Faulkner, C. J. Echeverri, and R. B. Vallee. Colocalization of cytoplasmic dynein with dynactin and CLIP-170 at microtubule distal ends. J Cell Sci, 112 ( Pt 10):1437-47, 1999.

T. L. Vincent, P. J. Green, and D. N. Woolfson. LOGICOIL-multi-state prediction of coiled-coil oligomeric state. Bioinformatics, 29(1):69-76, Jan. 2013.

I. Visiers, B. B. Braunheim, and H. Weinstein. Prokink: a protocol for numerical evaluation of helix distortions by proline. Protein Eng., 13(9):603-606, Jan. 2000.

J. Walshaw and D. N. Woolfson. Socket: a program for identifying and analysing coiled-coil motifs within protein structures. J. Mol. Biol., 307(5):1427-1450, Apr. 2001.

C. M. Waterman-Storer, S. Karki, and E. L. Holzbaur. The p150Glued component of the dynactin complex binds to both microtubules and the actin-related protein centractin (arp-1). Proc Natl Acad Sci U S A, 92(5):1634-1638, 1995.

A. A. Watson and C. A. O'Callaghan. Crystallization and x-ray diffraction analysis of human CLEC-2. Acta Crystallographica Section F Structural Biology and Crystallization Communications, 61(12):1094-1096, Dec. 2005.

B. Wickstead and K. Gull. Dyneins across eukaryotes: a comparative genomic analysis. Traffic, 8(12):1708-1721, Dec. 2007.

J. C. Williams, P. L. Roulhac, A. G. Roy, R. B. Vallee, M. C. Fitzgerald, and W. A. Hendrickson. Structural and thermodynamic characterization of a cytoplasmic dynein 
light chain-intermediate chain complex. Proc Natl Acad Sci U S A, 104(24):1002810033, June 2007.

B. Yang, Y.-J. Wu, M. Zhu, S.-B. Fan, J. Lin, K. Zhang, S. Li, H. Chi, Y.-X. Li, H.-F. Chen, S.-K. Luo, Y.-H. Ding, L.-H. Wang, Z. Hao, L.-Y. Xiu, S. Chen, K. Ye, S.-M. He, and M.-Q. Dong. Identification of cross-linked peptides from complex samples. Nat. Methods, 9(9):904-906, Sept. 2012.

Z. Yang, U. S. Tulu, P. Wadsworth, and C. L. Rieder. Kinetochore dynein is required for chromosome motion and congression independent of the spindle checkpoint. Curr. Biol., 17(11):973-980, June 2007.

T.-Y. Yeh, A. K. Kowalska, B. R. Scipioni, F. K. Y. Cheong, M. Zheng, U. Derewenda, Z. S. Derewenda, and T. A. Schroer. Dynactin helps target polo-like kinase 1 to kinetochores via its left-handed beta-helical p27 subunit. EMBO J., 32(7):10231035, Apr. 2013.

M. M. Young, N. Tang, J. C. Hempel, C. M. Oshiro, E. W. Taylor, I. D. Kuntz, B. W. Gibson, and G. Dollinger. High throughput protein fold identification by using experimental constraints derived from intramolecular cross-links and mass spectrometry. Proc. Natl. Acad. Sci. U.S.A., 97(11):5802-5806, May 2000.

R. H. Yun, A. Anderson, and J. Hermans. Proline in $\alpha$-helix: Stability and conformation studied by dynamics simulation. Proteins, 10(3):219-228, Mar. 1991.

J. Zhang, X. Yao, L. Fischer, J. F. Abenza, M. A. Peñalva, and X. Xiang. The p25 subunit of the dynactin complex is required for dynein-early endosome interaction. The Journal of Cell Biology, 193(7):1245 -1255, June 2011. 


\section{A. Composition of solutions}

\section{A.1. SDS-PAGE}

For the separation of differently sized proteins SDS-PAGE gels were used (modified after Laemmli (1970)). Depending on the size of the proteins to be separated the percentage of acrylamide was varied. Low percentage gels are needed for high molecular weight proteins, whereas high percentage gels give a better resolution for smaller proteins.

Table A.1.: Different SDS-PAGE gel compositions

\begin{tabular}{l|lll|l} 
& \multicolumn{3}{|c}{ Separating gel } & Stacking gel \\
\hline \hline & $12.5 \%$ & $15 \%$ & $17.5 \%$ & $4 \%$ \\
\hline Rotiphorese(R) Gel 30 (37.5:1) & $4.2 \mathrm{ml}$ & $5 \mathrm{ml}$ & $5.9 \mathrm{ml}$ & $0.67 \mathrm{ml}$ \\
Buffer $^{*}$ & $2.5 \mathrm{ml}$ & $2.5 \mathrm{ml}$ & $2.5 \mathrm{ml}$ & $1.25 \mathrm{ml}$ \\
$10 \%$ SDS-solution & $100 \mu \mathrm{l}$ & $100 \mu \mathrm{l}$ & $100 \mu \mathrm{l}$ & $50 \mu \mathrm{l}$ \\
TEMED & $10 \mu \mathrm{l}$ & $10 \mu \mathrm{l}$ & $10 \mu \mathrm{l}$ & $5 \mu \mathrm{l}$ \\
$10 \%$ Ammonium persulfate & $100 \mu \mathrm{l}$ & $100 \mu \mathrm{l}$ & $100 \mu \mathrm{l}$ & $35 \mu \mathrm{l}$ \\
$\mathrm{dH}_{2} \mathrm{O}$ & $3.09 \mathrm{ml}$ & $2.29 \mathrm{ml}$ & $1.39 \mathrm{ml}$ & $3 \mathrm{ml}$
\end{tabular}

* Buffers vary depending on the gel. Separating gel: For 12.5 and $15 \%$ gels $1.5 \mathrm{M}$ Tris buffer ( $\mathrm{pH} 8.8$ ) was used, whereas for $17.5 \%$ gels $2 \mathrm{M}$ Tris buffer ( $\mathrm{pH} 8.8$ ) was needed to get a better resolution for small proteins. Stacking gels: Here,the buffer was composed of $0.5 \mathrm{M}$ Tris at $\mathrm{pH} 6.8$.

Gels were cast between two glass plates (BIORAD). First the separating gel was poured between the plates and covered with water until it was polymerized. Then, the water was removed from the separating gel, before being covered with the stacking gel. A plastic comb was quickly put in the stacking gel to create pockets for the protein samples.

Gels were put into a electrophoresis system (BIORAD) and the container filled with running buffer. Protein samples were mixed with SDS sample buffer and applied into 
the pockets of the gel.

$10 \times$ Running Buffer:

30.3 g Tris-base

144.0 g glycine

10.0 g SDS

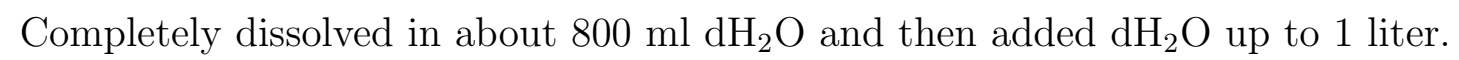

\section{A.2. SDS sample buffer}

The SDS sample buffer was added to the protein samples to denature their secondary structure and making them negatively charged. Due to the bromophenol blue dye a tracking of the sample through the SDS gels was possible. The sample buffer was diluted 1:5 with the protein sample.

6× Sample buffer:

0.131 M Tris-HCl, pH 6.8

$10 \%$ SDS

$0.05 \%$ Bromophenol blue

$50 \%$ Glycerol

$0.5 \mathrm{M} \mathrm{DTT}$

\section{A.3. Coomassie staining}

SDS-PAGE gels were stained with a Coomassie staining solution to visualise protein bands (Fairbanks et al., 1971). To do so, the gel was placed into a small plastic box, covered with the staining solution and put in the microwave for 30 to $90 \mathrm{sec}$ on high power. Then, the staining solution was removed and the gel was washed with water several times to remove excess Coomassie solution. Finally, the gel was covered in destaining solution and placed in a microwave for 3 to 4 min on high power to visualise the bands. 
Table A.2.: Buffers for SDS-PAGE staining

Coomassie staining solution

2.2 g Coomassie Brilliant Blue G250 (Roth)

$100 \mathrm{ml}$ Acetic acid

$250 \mathrm{ml}$ Isopropyl alcohol

$650 \mathrm{ml}$ Water

\section{Destaining solution}

$10 \%$ Acetic acid

\section{A.4. Determining protein concentration with Bradford}

In order to determine the protein concentration (Bradford, 1976) a calibration line using varying BSA (bovine serum albumin) concentrations $(0.2-1 \mathrm{mg} / \mathrm{ml}$ ) was prepared. $20 \mu \mathrm{l}$ of each concentration was mixed with $980 \mu \mathrm{l}$ Bradford solution, incubated for $5 \mathrm{~min}$ and measured at $595 \mathrm{~nm}$. Each concentration was measured three times. The absorbance was plotted against the BSA concentration and the equation of the line was determined. The equation was rearranged to solve unknown concentrations.

For the determination of the concentration of a protein $0.5-2 \mu \mathrm{l}$ of the sample were mixed with water to a total volume of $20 \mu \mathrm{l}$. This was added to $980 \mu \mathrm{l}$ Bradford solution and incubated for $5 \mathrm{~min}$. The absorbance was measured at a wavelength of $595 \mathrm{~nm}$ and then together with the dilution factor implemented into the rearranged equation to calculate the protein concentration.

\section{Table A.3.: Composition of Bradford solution}

100 mg Coomassie Brilliant Blue G250 (Roth)

$50 \mathrm{ml}$ Ethanol

then the following solutions were added:

$100 \mathrm{ml} \mathrm{85 \%}$ Phosphoric acid

$850 \mathrm{ml}$ Water $\left\{\begin{array}{l}\text { dissolved over night and } \\ \text { filtered through a filter paper }\end{array}\right.$ 



\section{B. Plasmid map}

The plasmid pRSFmD1mako is used for tandem gene expression. It consists of two multiple cloning sites (MCS) each having a 6x-His tag and a T7-promoter. For selection it has a kanamycine resistance cassette.

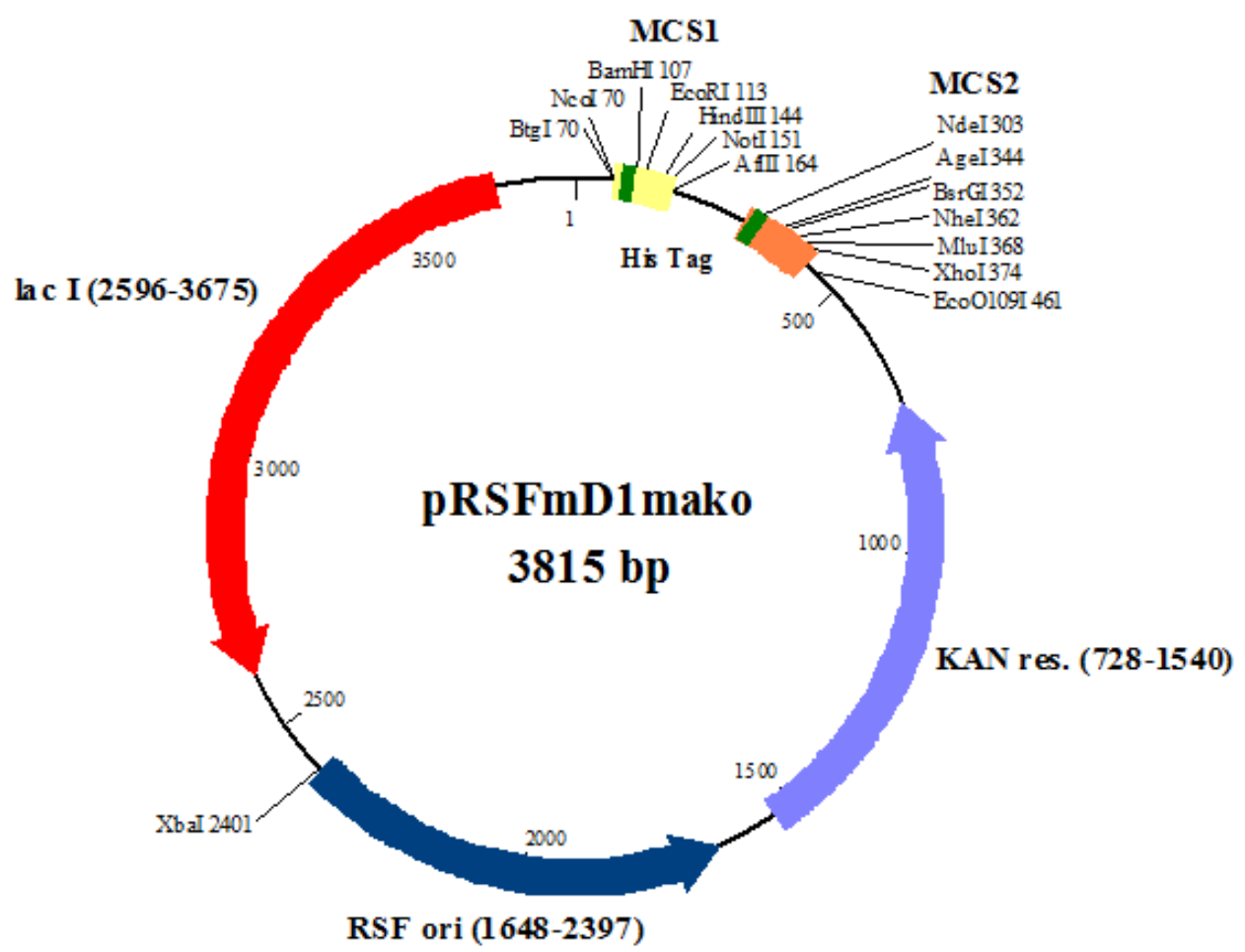

Figure B.1.: pRSFmD1mako vector map 



\section{Additional result files}

\section{C.1. Helical wheel representation of DCTN1 $1_{412-533}$}

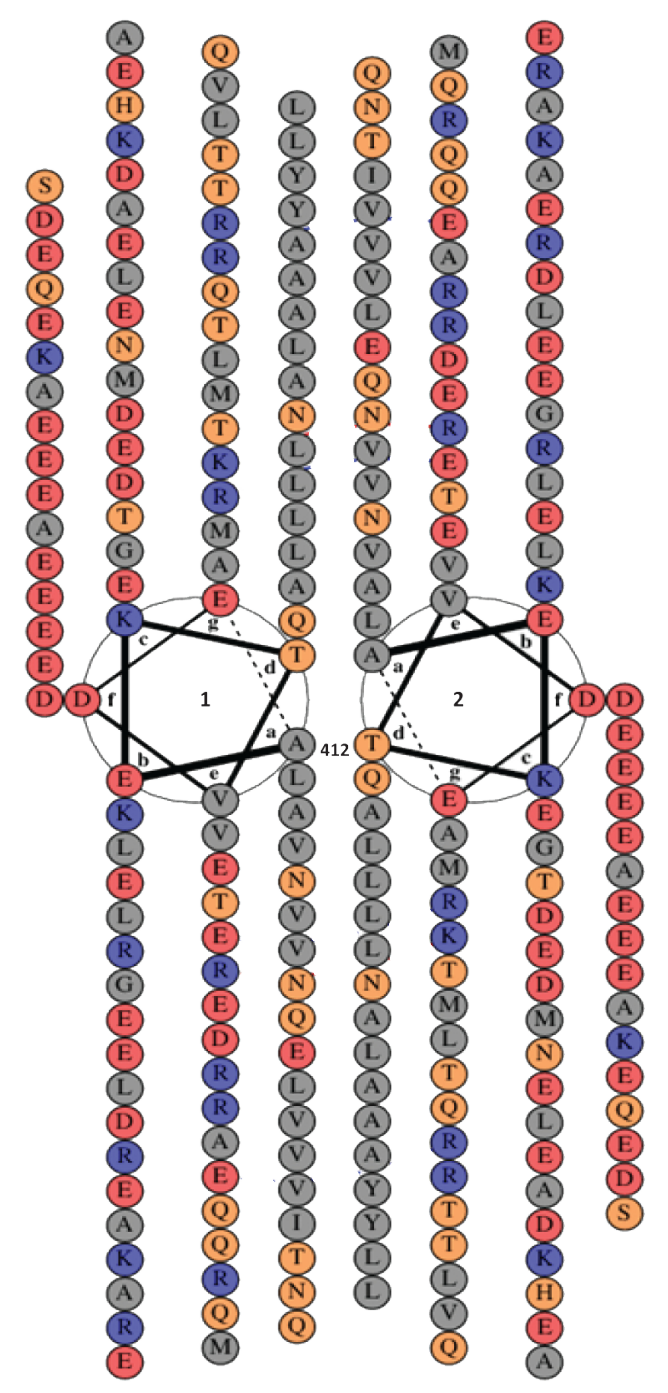

Figure C.1.: Helical wheel representation of $\mathrm{DCTN}_{1} 1_{42-533}$. Charged amino acids are coloured in red (acidic) and blue (alkaline). Polar, uncharged amino acids are coloured in orange. Created with DrawCoil 1.0 (http: //www.grigoryanlab.org/drawcoil/). 


\section{C.2. Example fragmentation spectra from cross-linking experiments}

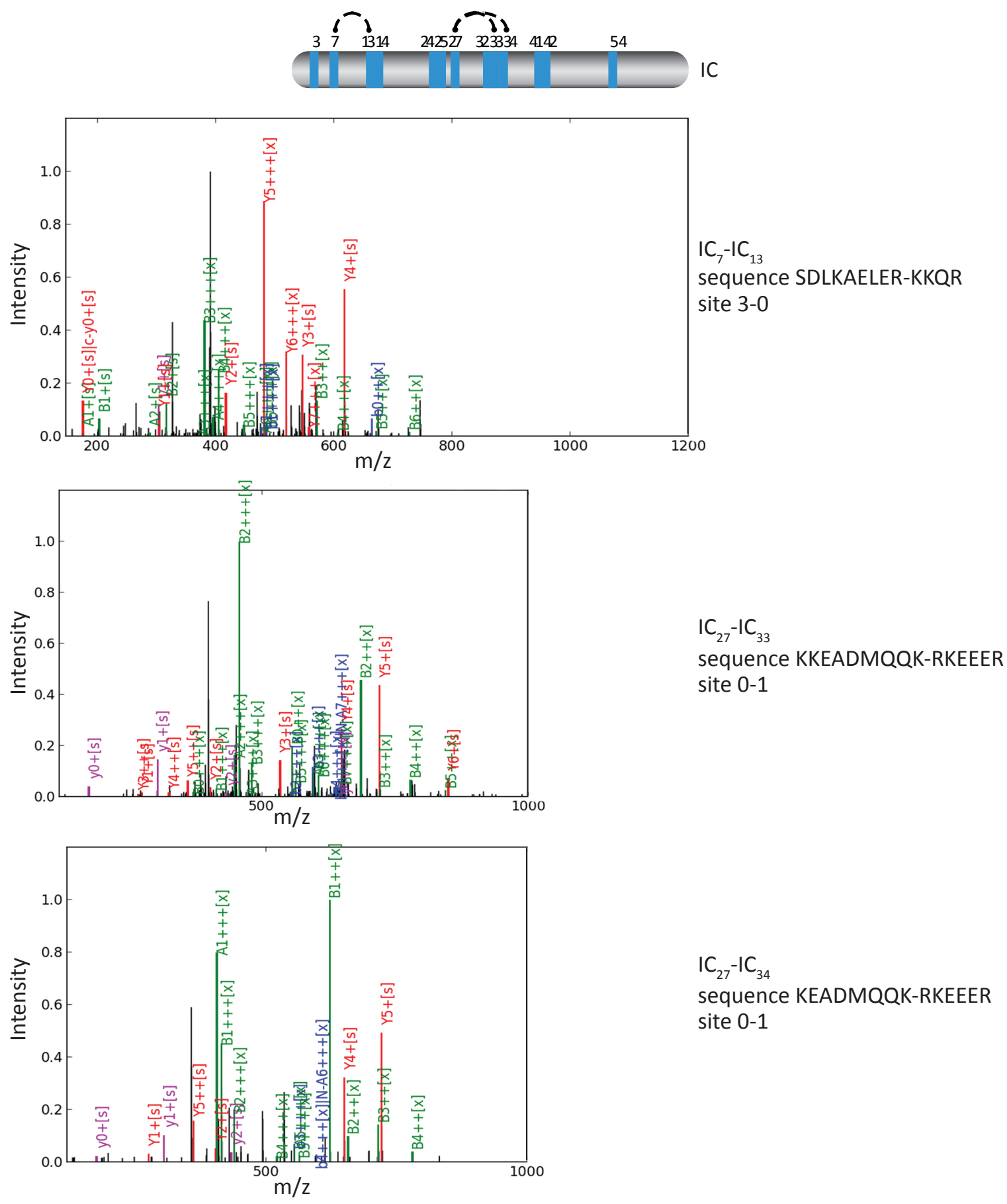

Figure C.2.: Intramolecular cross-links found in $\mathrm{IC}_{2-69}$. For each found crosslink an example fragmentation spectra is shown. Next to each spectra the cross-linked lysines are listed together with the peptide sequences and the cross-link site. 


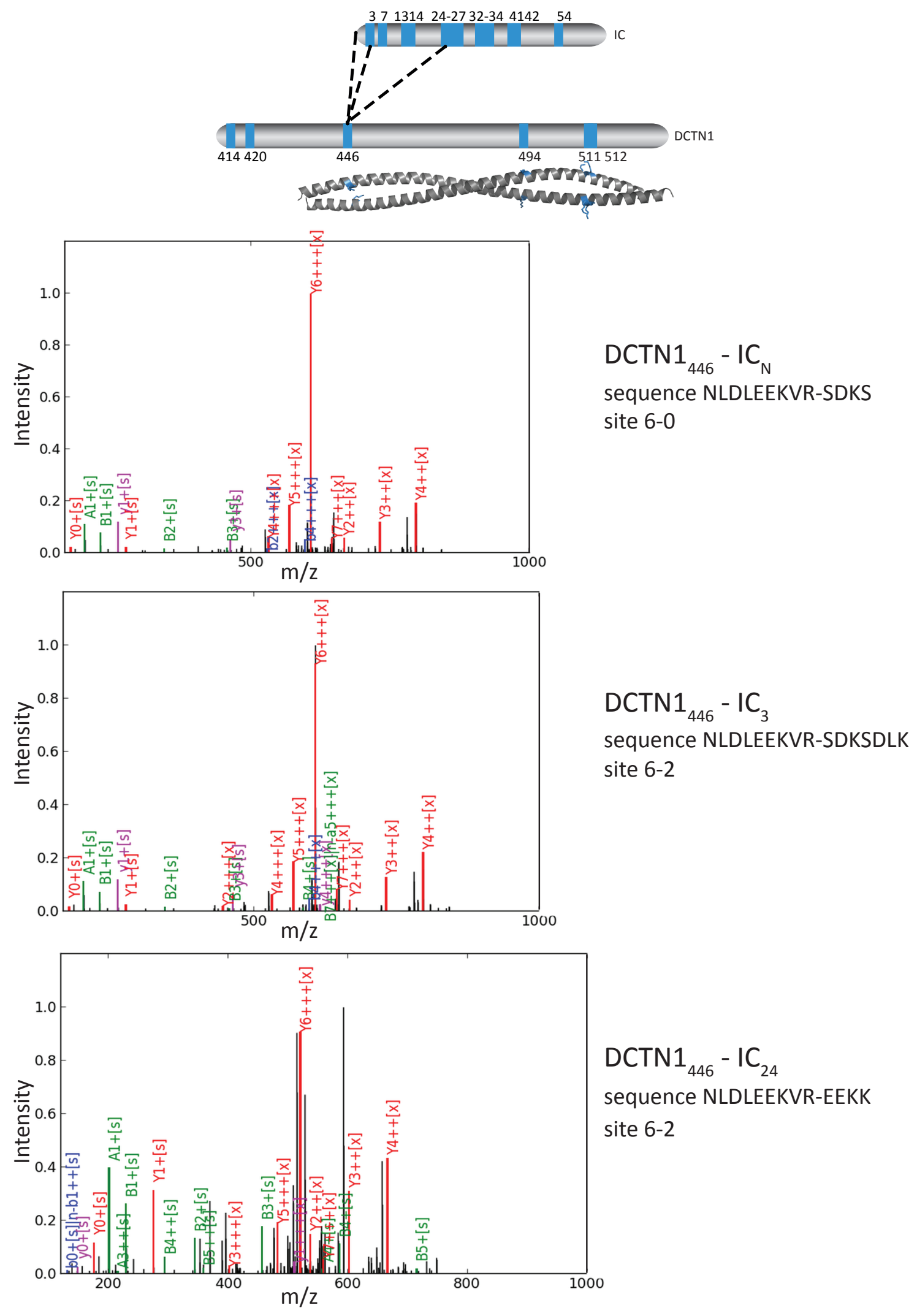

Figure C.3.: Intermolecular cross-links found between IC and the Nterminal DCTN1 $\mathbf{1}_{\mathbf{4 1 2}-\mathbf{5 3 3}}$. For each found cross-link an example fragmentation spectra is shown. Next to each spectra the cross-linked lysines are listed together with the peptide sequences and the cross-link site. 

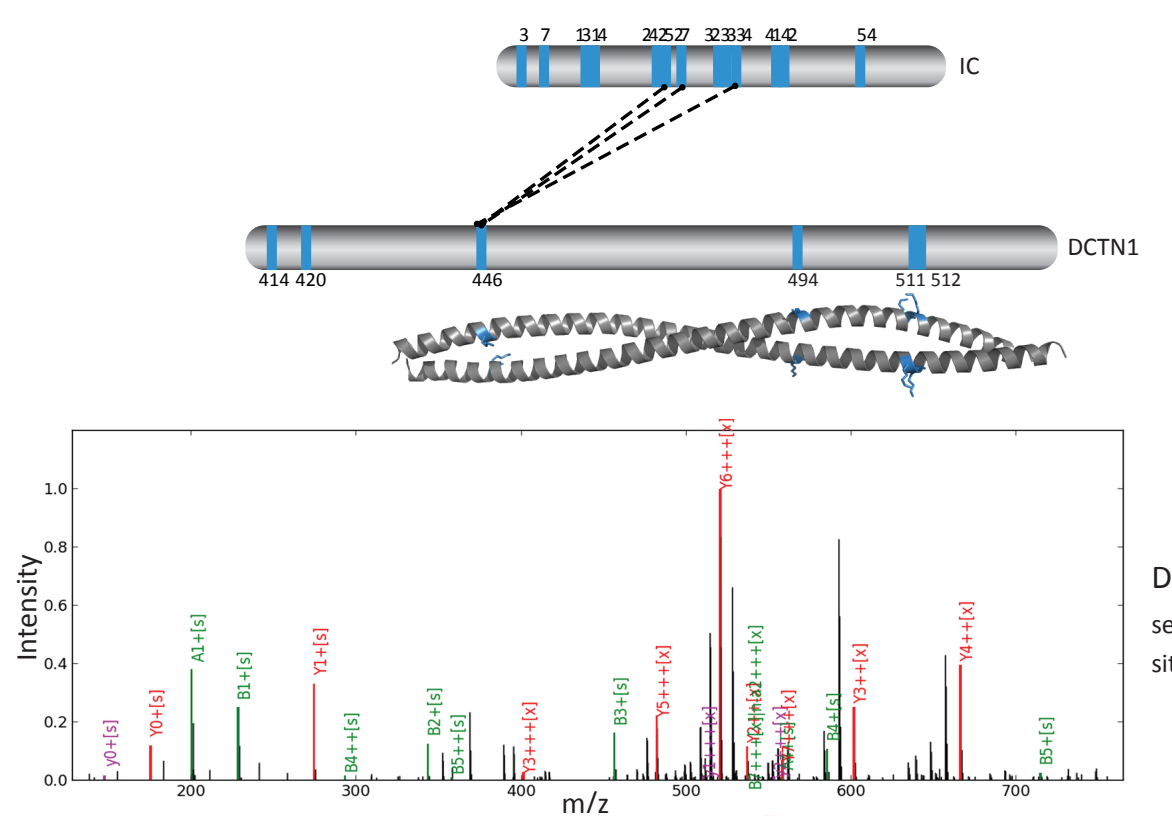

DCTN1 $_{446}-$ IC $_{25}$ sequence NLDLEEKVR-EEKK site 6-2

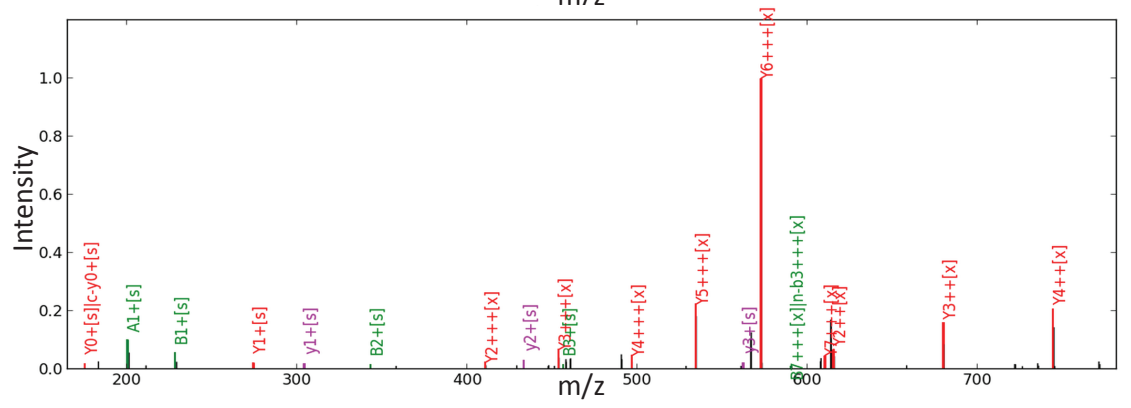

$\mathrm{DCTN1}_{446}-\mathrm{IC}_{27}$ sequence NLDLEEKVR-KEEER site 6-0

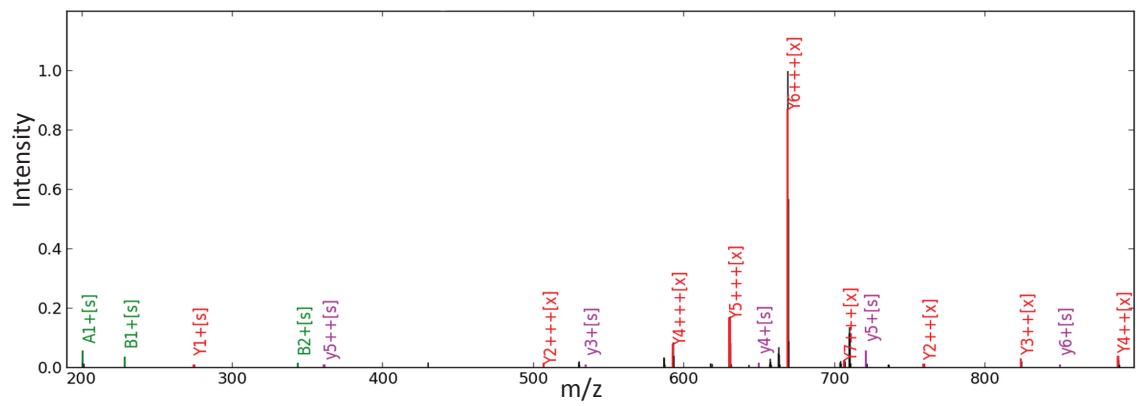
DCTN1 $_{446}-$ IC $_{34}$ sequence NLDLEEKVR-KEADMQQK site 6-0

Figure C.4.: Intermolecular cross-links found between IC and the middle section of DCTN1 $1_{412-533}$. For each found cross-link an example fragmentation spectra is shown. Next to each spectra the cross-linked lysines are listed together with the peptide sequences and the cross-link site. 

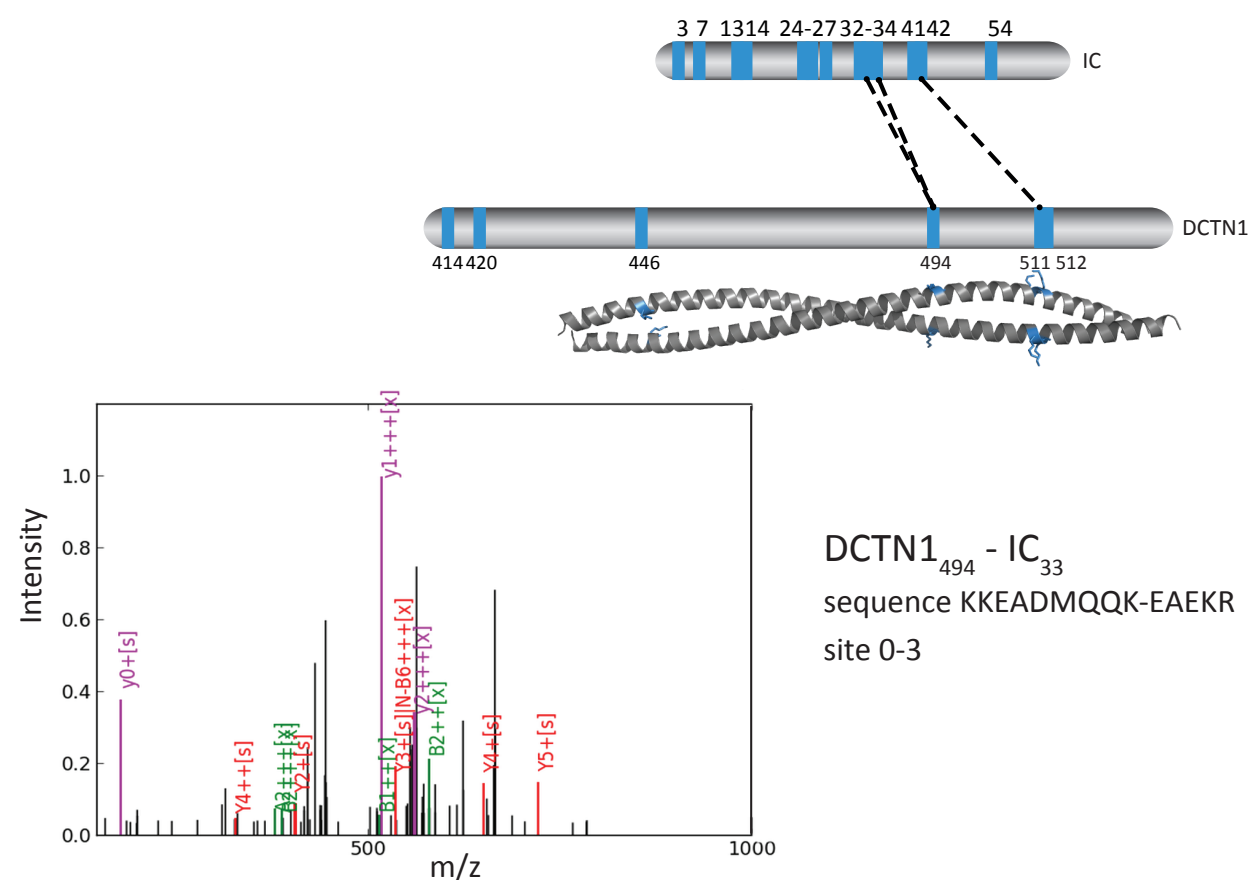

DCTN1 $_{494}-$ IC $_{33}$

sequence KKEADMQQK-EAEKR

site $0-3$

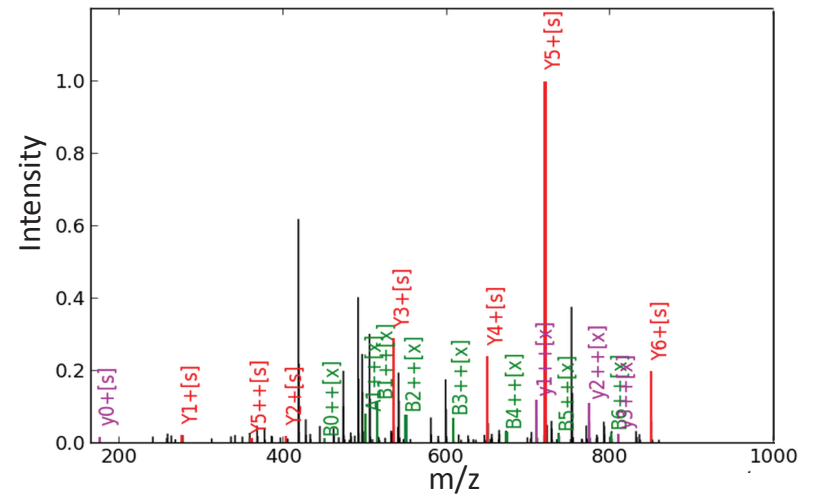

DCTN1 $_{494}-$ IC $_{34}$ sequence KEADMQQK-EAEKR

site $0-3$

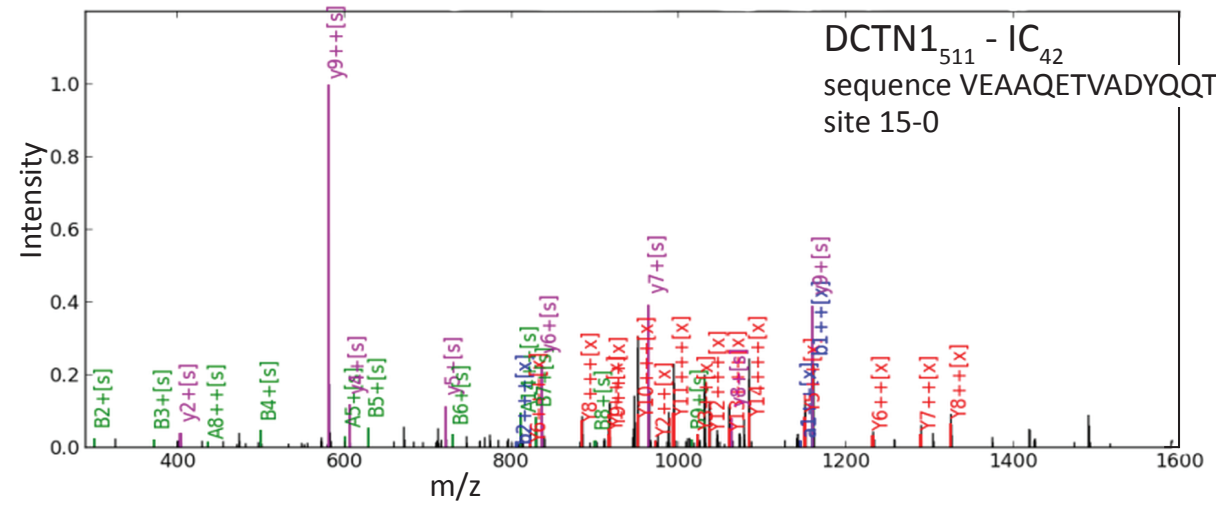

Figure C.5.: Intermolecular cross-links found between IC and the Cterminal DCTN1 $\mathbf{1}_{\mathbf{4 1 2 - 5 3 3}}$. For each found cross-link an example fragmentation spectra is shown. Next to each spectra the cross-linked lysines are listed together with the peptide sequences and the cross-link site. 



\section{Acknowledgment}

Hereby I thank all the people who contributed directly or indirectly to my PhD thesis.

First of all, I would like to thank Dr. Martin Kollmar for proposing this fascinating project, introducing me to the subject and giving valuable suggestions throughout my work.

A special thanks to Prof. Christian Griesinger for giving me the opportunity to work in the department of "NMR-based structural biology" and for providing excellent working conditions.

Furthermore, I would like to thank my thesis committee members, Prof. Reinhard Lührmann and Dr. Hans Dieter Schmitt, for helpful discussion and feedback.

I want to thank Dr. Christian Eckert for helping me familiarize with protein purification and crystallization, for producing the first crystals, and for helpful discussions.

Chung-Tien Lee for helping me with the performance and analysis of the cross-linking experiments.

Dr. Tim Grüne for help with $S H E L X C / D / E$ to solve the phases.

Dr. Björn Hammesfahr, Dr. Klas Hatje, and Benjamin Schomburg for proof-reading the manuscript and giving helpful comments.

Stefanie Mühlhausen for her help with everything related to computers, Dominic Simms for WaggaWagga, and Agniezka Goretzki and Christin Kellner for help and laughter in the lab.

Dr. Jan Keller-Findeisen for proof-reading the manuscript, help with $\mathrm{ET}_{\mathrm{E} X} \mathrm{X}$, and for being the best moral support I could ask for.

The whole NMR II department, especially the people from the chemistry lab and the technical assistants from the biology lab, for valuable ideas and for being what they are - good company.

Zum Schluß möchte ich mich auch ganz doll bei meinen Eltern bedanken, die meine Ausbildung ermöglicht und mich immer unterstützt haben. 